\title{
THE STRENGTHENING AND WEAKENING INSTRUMENT: COMPARABILITY OF TOPOLOGIES SPACES
}

\author{
B. Dvalishvili
}

\begin{abstract}
Along with noncomparable topologies, the paper concentrates on situations, where in a bitopological space one topology is finer than the other, which is frequently encountered in applications. In this context, different families of sets are considered and the bitopological modification of the Cantor-Bendixson theorem is proved. The three operators are defined, which characterize the degrees of nearness of the four boundaries of any set, tangency of topologies, $S$-, $C$ - and $N$-relations, and thus make it possible to compare small inductive dimensions at some special point. Furthermore, different properties of pairwise small and pairwise large inductive dimensions are studied. In the final part, the conditions are given, under which a bitopological space preserves the property to be an $(i, j)$-Baire space to the image and preimage. Relations between pairwise small and large inductive dimensions of the domain and the range of a $d$-closed and $d$-continuous function are investigated.
\end{abstract}

\section{Introduction}

J. C. Kelly defined a bitopological space $\left(X, \tau_{1}, \tau_{2}\right)$ to be a set $X$ with two topologies $\tau_{1}$ and $\tau_{2}$ on it [15].

In studying various kinds of bitopologies, i.e., ordered pairs of topologies on a set, this paper concentrates on situations, where one of the topologies is finer than the other, which is typical of applications of the theory of bitopological spaces.

Throughout the paper the following abbreviations are used: BS for a bitopological space, BsS for a bitopological subspace (the plural form for all abbreviated nouns is 's) and the symbols $\mathbb{N}, \mathbb{Z}, \mathbb{Q}$ and $\mathbb{R}$ for sets of all

AMS (MOS) Subject Classification 1991. Primary: 54E55.

Key words and phrases: indicator of nearness, $(i, j)$-dense in itself set, $(i, j)$-perfect set, $(i, j)$-scattered set, $(i, j)$-Baire space, almost $(i, j)$-Baire space, $(i, j)$-small inductive dimension, $(i, j)$-large inductive dimension. 
natural numbers (excluding zero), all integers, all rational numbers and all real numbers, respectively. Always $i, j \in\{1,2\}$ and $i \neq j$. Given a family $\mathcal{A}=\left\{A_{s}\right\}_{s \in S} \subset 2^{X}$, where $2^{X}$ is the power set of $X$, let co $\mathcal{A}$ denote the conjugate family co $\mathcal{A}=\left\{X \backslash A_{s}: A_{s} \in \mathcal{A}\right\}$. Further, the cardinal number $|X|$, called the cardinality of $X$, is assigned to each set $X$ and the cardinal number assigned to the set of all natural numbers is denoted by $\aleph_{0}$.

If $\left(X, \tau_{1}, \tau_{2}\right)$ is a $\mathrm{BS}$ and $\mathcal{P}$ is some topological property, then $(i, j) \mathcal{P}$ denotes the analogue of this property for $\tau_{i}$ with respect to $\tau_{j}$, and $p \mathcal{P}$ denotes the conjunction $(1,2) \mathcal{P} \wedge(2,1) \mathcal{P}$, i.e., $p-\mathcal{P}$ denotes the "absolute" bitopological analogue of $\mathcal{P}$, where $p$ is the abbreviation for "pairwise". As we will see below, sometimes $(1,2)-\mathcal{P} \Longleftrightarrow(2,1) \mathcal{P}$ (and thus $\Longleftrightarrow p-\mathcal{P})$ so that it suffices to consider one of these three bitopological analogues. Also note that $\left(X, \tau_{i}\right)$ has a property $\mathcal{P} \Longleftrightarrow\left(X, \tau_{1}, \tau_{2}\right)$ has a property $i \mathcal{P}$ and $d-\mathcal{P} \Longleftrightarrow 1 \mathcal{P} \wedge 2-\mathcal{P}$, where $d$ is the abbreviation for "double". By a BS $\left(X, \tau_{1}<\tau_{2}\right)$ is always be meant a $\mathrm{BS}\left(X, \tau_{1}, \tau_{2}\right)$ with $\tau_{1} \subset \tau_{2}$.

Let $\left(X, \tau_{1}, \tau_{2}\right)$ be any BS and $A \in 2^{X}$ be its any subset. Then $\tau_{i} \operatorname{cl} A$ and $\tau_{i}$ int $A$ denote respectively the closure and the interior of $A$ in the topology $\tau_{i}$. If $\mathcal{A}=\left\{A_{s}\right\}_{s \in S} \subset 2^{X}$ is any family, then $i$-Cl $\mathcal{A}=\left\{\tau_{i} \operatorname{cl} A_{s}\right\}_{s \in S}$. Furthermore, $p-\operatorname{Cl}(X)=\left\{A \in 2^{X}: A=\tau_{1} \operatorname{cl} A \cap \tau_{2} \operatorname{cl} A\right\}, i-\mathcal{B} d(X)=\left\{A \in 2^{X}: \tau_{i}\right.$-int $A=$ $\varnothing\}, i-\mathcal{D}(X)=\left\{A \in 2^{X}: \tau_{i} \operatorname{cl} A=X\right\}$ and $(i, j)-\mathcal{N} \mathcal{D}(X)=\left\{A \in 2^{X}:\right.$ $\tau_{i}$ int $\left.\tau_{j} \operatorname{cl} A=\varnothing\right\}$ are the families of all $p$-closed, $i$-boundary, $i$-dense and $(i, j)$-nowhere dense subsets of $X$, respectively.

Also note that here $i-\mathcal{F}_{\sigma}(X)=\left\{A \in 2^{X}: A\right.$ is an $i-F_{\sigma}$ set $\},(i, j)$ $\mathcal{S O}(X)=\left\{A \in 2^{X}:\right.$ there is a set $U \in \tau_{i}$ such that $\left.U \subset A \subset \tau_{j} \operatorname{cl} U\right\}$ and $(i, j)-\mathcal{C} a t g_{\mathrm{I}}(X)=\left\{A \in 2^{X}: A=\bigcup_{n=1}^{\infty} A_{n}, A_{n} \in(i, j)-\mathcal{N} \mathcal{D}(X), n=\overline{1, \infty}\right\}$, $(i, j)-\mathcal{C a t g}_{\mathrm{II}}(X)=2^{X} \backslash(i, j)-\mathcal{C} a t g_{\mathrm{I}}(X)$ and $X \in(i, j)-\mathcal{C a t g}_{\mathrm{I}}(X) \Longleftrightarrow X$ is of $(i, j)-\mathcal{C} a t g \mathrm{I}, X \in(i, j)-\mathcal{C}$ atg $g_{\mathrm{II}}(X) \Longleftrightarrow X$ is of $(i, j)-\mathcal{C}$ atg II.

In a $\mathrm{BS}\left(X, \tau_{1}, \tau_{2}\right)$ the following double indexation is used: $A_{i}^{d}=\{x \in$ $X: x$ is an $i$-accumulation point of $A\}$ and $A_{j}^{i}=\{x \in X: x$ is a $j$-isolated point of $A\}$, i.e., the lower indices $i$ and $j$ denote the belonging to the topology and therefore always $i, j \in\{1,2\}$, while the upper indices $d$ and $i$ are fixed as the accumulation and isolation symbols, respectively. Thus in a BS $\left(X, \tau_{1}, \tau_{2}\right)$ we have: $A_{j}^{i}=A \backslash A_{j}^{d}, A$ is a $j$-discrete set $\Longleftrightarrow A=A_{j}^{i}, A$ is a $j$-dense in itself set $\Longleftrightarrow A \subset A_{j}^{d}$ and $A$ is a $j$-perfect set $\Longleftrightarrow A=A_{j}^{d}$.

The paper consists of three paragraphs, of which $\S 1$ deals with the bitopological modifications of open and closed domains [16], and locally closed, dense in themslves, perfect and scattered sets (see, for example [17]). It contains the proof of the bitopological analogue of the well-known CantorBendixson theorem. Moreover, in a BS $\left(X, \tau_{1}<\tau_{2}\right)$ a special subfamily of the 
family $(2,1)-\mathcal{N D}(X)$ is chosen, the union of whose elements also belongs to $(2,1)-\mathcal{N} \mathcal{D}(X)$. At the end of the paragraph, three special operators are introduced and studied on $2^{X}$, which are used to determine exactly the degrees of nearness of the four boundaries of any set and give a simple characterization of the $<_{S^{-}},<_{C^{-}}$and $<_{N^{-}}$relations.

It should be mentioned that one of these operators also defines the tangency of topologies at a certain point of a BS $\left(X, \tau_{1}<\tau_{2}\right)$ in the sense of [2], which thus makes it possible, in addition to the results from [9], [10], to compare at this point the small inductive dimension functions 2 -ind $X$ and $(1,2)$-ind $X$, aas well as $(2,1)$-ind $X$ and 1 -ind $X$. In our opinion, it is interesting to apply the introduced operators in considering initial and fine topologies in the potential theory especially when a fine topology is compatible with a quasi topology in the sense of B. Fuglede [12] (see also [9], [11]).

The notion of a zero dimensional BS was introduced by I. L. Reilly [21] on the basis of the idea of bitopological disconnectedness considered by J. Swart [23]. A systematic study of bitopological dimension functions was undertaken by M. Jelić [13], [14], M. D. Ćirić [4] and by us [5]-[7]. As different from [4], [13], [14], the ideas set forth in [5]-[7] were essentially based on the notion of a bitopological boundary.

In $\S 2$, pairwise small and large inductive dimensions are formulated in terms of both bitopological partitions and neighbourhoods in a manner such that for $n=0$ a pairwise small inductive dimension leads to the notion of I. L. Reilly. Moreover, the analogues of the well-known sum theorem and the first decomposition theorem are proved for a pairwise small inductive dimension.

Furthermore, interrelations of pairwise inductive dimensions and their topological versions are considered when topologies are comparable by inclusion or are coupled, $<_{C}$-related, near and $<_{N}$-related. The sum theorem is also proved for a large inductive dimension and the conditions, under which pairwise small and large inductive dimensions coincide, are established.

In $\S 3$, the conditions are given, under which a BS preserves the property to be an $(1,2)$-Baire space to the image and preimage, and relations between pairwise small and large inductive dimensions of the domain and the range of a $d$-closed and $d$-continuous function are studied.

\section{Some Special Operators and Families of Sets in Bitopological Spaces}

The family $(i, j)-\mathcal{N D}(X)$ plays an important role not only in the definition of Baire-like properties, but also has interest of its own, especially when dealing with BS's of the type $\left(X, \tau_{1}<\tau_{2}\right)$. 
Definition 2.1. A subset $A$ of a $\mathrm{BS}\left(X, \tau_{1}, \tau_{2}\right)$ is $(i, j)$-nowhere dense $((i, j)$-boundary) at a point $x \in X$ if there exists an $i$-open neighborhood $U(x)$ such that $\tau_{i} \operatorname{int} \tau_{j} \operatorname{cl}(A \cap U(x))=\varnothing\left(\tau_{i} \operatorname{int} \tau_{j} \operatorname{int}(A \cap U(x))=\varnothing\right)$ [9].

For every point $x \in X$ the family of all sets, which are $(i, j)$-nowhere dense $((i, j)$-boundary) at the point $x$, is denoted by $(i, j)-\mathcal{N} \mathcal{D}(X, x)((i, j)$ $\mathcal{B} d(X, x))$.

In a BS $\left(X, \tau_{1}<\tau_{2}\right)$ the following inclusions and equality hold for every point $x \in X$ :

$$
\begin{gathered}
1-\mathcal{N D}(X, x) \subset(1,2)-\mathcal{N D}(X, x) \text { and }(2,1)-\mathcal{N D}(X, x) \subset 2-\mathcal{N D}(X, x), \\
(1,2)-\mathcal{B} d(X, x)=1-\mathcal{B} d(X, x) \subset(2,1)-\mathcal{B} d(X, x) \supset 2-\mathcal{B} d(X, x) .
\end{gathered}
$$

Proposition 2.1. Let $\left(X, \tau_{1}<\tau_{2}\right)$ be $a \mathrm{BS}$ and $x \in X$ any point. Then $A \in(1,2)-\mathcal{N D}(X, x) \Longleftrightarrow \tau_{2} \operatorname{cl} A \in 1-\mathcal{B} d(X, x)$.

Proof. If $A \bar{\epsilon}(1,2)-\mathcal{N} \mathcal{D}(X, x)$, then $\tau_{1}$ int $\tau_{2} \operatorname{cl}(A \cap U(x)) \neq \varnothing$ for every 1-open neighborhood $U(x)$. Therefore there exists a set $V \in \tau_{1} \backslash\{\varnothing\}$ such that $V \subset \tau_{2} \operatorname{cl}(A \cap U(x))$. The inclusion $\tau_{1} \subset \tau_{2}$ implies $V=V \cap \tau_{2} \operatorname{cl}(A \cap U(x)) \subset$ $\tau_{2} \operatorname{cl}(V \cap A \cap U(x))$, where $V \cap U(x) \subset \tau_{2} \operatorname{cl}(A \cap U(x)) \cap U(x) \subset \tau_{2} \operatorname{cl}(A \cap U(x))$. Thus $\tau_{1} \operatorname{int}\left(\tau_{2} \operatorname{cl} A \cap U(x)\right) \neq \varnothing$ so that $\tau_{2} \operatorname{cl} A \cap U(x) \bar{\epsilon} 1-\mathcal{B} d(X)$ and, since $U(x) \in \tau_{1}$ is an arbitrary neighborhood, we obtain $\tau_{2} \operatorname{cl} A \bar{\in} 1-\mathcal{B} d(X, x)$.

Conversely, let $\tau_{2} \operatorname{cl} A \bar{\in} 1-\mathcal{B} d(X, x)$. Then $\tau_{1} \operatorname{int}\left(\tau_{2} \operatorname{cl} A \cap U(x)\right) \neq \varnothing$ for every 1-open neighborhood $U(x)$. Hence there is a set $V \in \tau_{1} \backslash\{\varnothing\}$ such that $V \subset \tau_{2} \operatorname{cl} A \cap U(x) \subset \tau_{2} \operatorname{cl}(A \cap U(x))$. Therefore $\tau_{1} \operatorname{int} \tau_{2} \operatorname{cl}(A \cap U(x)) \neq \varnothing$ and, since $U(x) \in \tau_{1}$ is an arbitrary neighborhood, we obtain $A \bar{\in}(1,2)-\mathcal{N D}(X, x)$.

At this point it should additionally be said that in a $\operatorname{BS}\left(X, \tau_{1}<\tau_{2}\right)$ the inclusion $U \cap \tau_{1} \operatorname{cl} A \subset \tau_{1} \operatorname{cl}(U \cap A)$ is not correct for any sets $U \in \tau_{2}$ and $A \subset X$ as is demonstrated by the following simple example: $X=\{a, b, c, d\}$, $\tau_{1}=\{\varnothing,\{a, b\}, X\}, \tau_{2}$ is the discrete topology on $X, U=\{c\} \in \tau_{2}$ and $A=\{b, d\}$.

Thus we find that the analogue of the latter proposition does not, generally speaking, hold for the $(2,1)$-case.

Corollary. In a $\mathrm{BS}\left(X, \tau_{1}<\tau_{2}\right)$ the following conditions are satisfied:

(1) $\{x \in X: A \bar{\epsilon}(1,2)-\mathcal{N D}(X, x)\}=\tau_{1} \operatorname{cl} \tau_{1} \operatorname{int} \tau_{2} \operatorname{cl} A$.

(2) $\{x \in A: A \in(1,2)-\mathcal{N D}(X, x)\} \in(1,2)-\mathcal{N D}(X)$.

Proof. (1) Using Theorem 2 from [17, p. 78], we obtain $\{x \in X$ : $\left.\tau_{2} \mathrm{cl} A \bar{\in} 1-\mathcal{B} d(X, x)\right\}=\tau_{1} \operatorname{cl} \tau_{1}$ int $\tau_{2} \mathrm{cl} A$. It remains to use Proposition 2.1.

(2) It is clear that $\{x \in A: A \in(1,2)-\mathcal{N D}(X, x)\}=A \backslash \tau_{1} \operatorname{cl} \tau_{1}$ int $\tau_{2} \mathrm{cl}$ $A \subset \tau_{2} \operatorname{cl} A \backslash \tau_{1} \operatorname{int} \tau_{2} \operatorname{cl} A$ and $\tau_{1} \operatorname{int}\left(\tau_{2} \operatorname{cl} A \backslash \tau_{1} \operatorname{int} \tau_{2} \operatorname{cl} A\right)=\tau_{1} \operatorname{int} \tau_{2} \operatorname{cl} A \cap\left(X \backslash \tau_{1}\right.$ $\operatorname{cl} \tau_{1}$ int $\left.\tau_{2} \operatorname{cl} A\right)=\varnothing$. Therefore $\tau_{2} \operatorname{cl} A \backslash \tau_{1}$ int $\tau_{2} \operatorname{cl} A \in \operatorname{co} \tau_{2} \cap 1-\mathcal{B} d(X) \subset(1,2)$ $\mathcal{N D}(X)$. 
In particular, if $A$ is $(1,2)$-nowhere dense at each of its points, then $A=A \backslash \tau_{1} \operatorname{cl} \tau_{1}$ int $\tau_{2} \operatorname{cl} A$ so that $\tau_{1}$ int $\tau_{2} \operatorname{cl} A=\varnothing \Longleftrightarrow A \in(1,2)-\mathcal{N D}(X)$.

Definition 2.2. A family $A=\left\{\mathcal{A}_{s}\right\}_{s \in \mathcal{S}}$ of subsets in a BS $\left(X, \tau_{1}, \tau_{2}\right)$ is $i$-locally finite at a $j$-dense set of points of $X$ if for every set $U \in \tau_{i} \backslash\{\varnothing\}$ there exists a set $V \in \tau_{j} \backslash\{\varnothing\}$ such that $V \subset U$ and $\left\{s \in S: V \cap A_{s} \neq \varnothing\right\}$ is finite.

Clearly, if $A=\left\{\mathcal{A}_{s}\right\}_{s \in \mathcal{S}}$ is $i$-locally finite at a $j$-dense set of points of $X$, then the family $j$ - $\mathrm{Cl} \mathcal{A}$ is also $i$-locally finite at a $j$-dense set of points of $X$.

Theorem 2.1. If a family $A=\left\{\mathcal{A}_{s}\right\}_{s \in \mathcal{S}} \subset(2,1)-\mathcal{N D}(X)$ is 2-locally finite at a 1-dense set of points of a $\mathrm{BS}\left(X, \tau_{1}<\tau_{2}\right)$, then $\cup_{s \in S} A_{s} \in(2,1)-\mathcal{N D}(X)$.

Proof. Let $A=\left\{\mathcal{A}_{s}\right\}_{s \in \mathcal{S}}$ be 2-locally finite at a 1-dense set of points of $X$. Then 1-Cl $A=\left\{\tau_{1} \mathrm{cl} A_{s}\right\}_{s \in S}$ must also be 2-locally finite at a 1-dense set of points of $X$ so that for every set $U \in \tau_{2} \backslash\{\varnothing\}$ there exists a set $V \in \tau_{1} \backslash\{\varnothing\}$ such that $V \subset U$ and $\left\{s_{k} \in S: V \cap \tau_{1} \mathrm{cl} A_{s_{k}} \neq \varnothing\right\}$ is finite. Let $\left\{\tau_{1} \mathrm{cl} A_{s_{k}}\right\}_{k=1}^{n}$ be the corresponding finite family. Since $\tau_{1} \mathrm{cl} A_{s_{k}} \in(2,1)-\mathcal{N D}(X)$ for every $k=\overline{1, n}$, we have $X \backslash \tau_{1} \operatorname{cl} A_{s_{k}} \in \tau_{1} \cap 2-\mathcal{D}(X)$ for every $k=\overline{1, n}$. One can easily verify that $W=V \cap\left(\cap_{k=1}^{n}\left(X \backslash \tau_{1} \operatorname{cl} A_{s_{k}}\right)\right) \neq \varnothing$. Contrary, let $W=\varnothing$ so that $V \subset$ $X \backslash\left(\cap_{k=1}^{n}\left(X \backslash \tau_{1} \operatorname{cl} A_{s_{k}}\right)\right)=\bigcup_{k=1}^{n} \tau_{1} \operatorname{cl} A_{s_{k}}$. In that case $\varnothing \neq \tau_{1}$ int $\bigcup_{k=1}^{n} \tau_{1} \operatorname{cl} A_{s_{k}}=$ $\tau_{1}$ int $\tau_{1} \mathrm{cl} \bigcup_{k=1}^{n} A_{s_{k}} \subset \tau_{2}$ int $\tau_{1} \mathrm{cl} \bigcup_{k=1}^{n} A_{s_{k}}$, which is impossible since, by Corollary 2 of Proposition 2.1.3 in [11], $\bigcup_{k=1}^{n} A_{s_{k}} \in(2,1)-\mathcal{N} \mathcal{D}(X)$. Hence $W \in \tau_{1} \backslash\{\varnothing\}$. On the other hand, $V \cap \tau_{1} \operatorname{cl} A_{s}=\varnothing$ for $s \neq s_{k}$ so that $V \subset X \backslash \tau_{1} \operatorname{cl} A_{s}$ for every $s \neq s_{k}$ and consequently the set $W \subset V$ satisfies the inclusion $W \subset \bigcap_{s \in S}\left(X \backslash \tau_{1} \operatorname{cl} A_{s}\right)=X \backslash \cup_{s \in S} \tau_{1} \operatorname{cl} A_{s}$. Hence $W \in \tau_{1} \backslash\{\varnothing\}$ implies $W \subset$ $\tau_{1} \operatorname{int}\left(X \backslash \cup_{s \in S} \tau_{1} \operatorname{cl} A_{s}\right)=X \backslash \tau_{1} \operatorname{cl} \cup_{s \in S} \tau_{1} \operatorname{cl} A_{s} \subset X \backslash \tau_{1} \operatorname{cl} \cup_{s \in S} A_{s}$. But $W \subset V \subset U$ and therefore $U \cap\left(X \backslash \tau_{1} \operatorname{cl} \cup_{s \in S} A_{s}\right) \neq \varnothing$ so that $\tau_{2} \operatorname{cl}\left(X \backslash \tau_{1} \operatorname{cl} \cup_{s \in S} A_{s}\right)=X$ since $U \in \tau_{2} \backslash\{\varnothing\}$ is an arbitrary set. Thus $\cup_{s \in S} A_{s} \in(2,1)-\mathcal{N} \mathcal{D}(X)$.

Definition 2.3. A subset $A$ of a $\operatorname{BS}\left(X, \tau_{1}, \tau_{2}\right)$ is $(i, j)$-locally closed at its point $x$ if there exists a set $U \in \tau_{i}$ such that $x \in U$ and $U \cap A=U \cap \tau_{j} \operatorname{cl} A$ $[9]$.

For every point $x \in X$ the family of all sets that are $(i, j)$-locally closed at the their common point $x$ is denoted by $(i, j)-\mathcal{L C}(X, x)$.

A subset $A$ of a $\mathrm{BS}\left(X, \tau_{1}, \tau_{2}\right)$ is $(i, j)$-locally closed if it is $(i, j)$-locally closed at each of its points. The family of all such subsets of $X$ is denoted by $(i, j)-\mathcal{L C}(X)$. 
In a BS $\left(X, \tau_{1}<\tau_{2}\right)$ the following inclusions hold for every point $x \in X$ :

$$
\begin{array}{ccc}
1-\mathcal{L C}(X, x) & \subset & (2,1)-\mathcal{L C}(X, x) \\
\cap & & \cap \\
(1,2)-\mathcal{L C}(X, x) & \subset & 2-\mathcal{L C}(X, x)
\end{array}
$$

and therefore

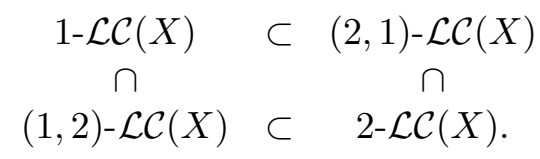

Theorem 2.2. Let $A$ be a subset of a BS $\left(X, \tau_{1}<\tau_{2}\right)$. Then the set $\{x \in A: A$ is not $(2,1)$-locally closed at a point $x\}$ coincides with the set $A \cap \tau_{2} \operatorname{cl}\left(\tau_{1} \operatorname{cl} A \backslash A\right)$.

Proof. First assume that $x \in A$ and $x \bar{\in} A \cap \tau_{2} \operatorname{cl}\left(\tau_{1} \operatorname{cl} A \backslash A\right)$, i.e., $x \bar{\epsilon} \tau_{2} \operatorname{cl}\left(\tau_{1} \operatorname{cl} A \backslash A\right)$. If $U=X \backslash \tau_{2} \operatorname{cl}\left(\tau_{1} \operatorname{cl} A \backslash A\right)$, then $x \in U$ and $U \cap\left(\tau_{1} \operatorname{cl} A \backslash A\right)=$ $\varnothing$ and so $\left(U \cap \tau_{1} \operatorname{cl} A\right) \backslash A=\varnothing$. Hence $\left(U \cap \tau_{1} \operatorname{cl} A\right) \cap(X \backslash A)=\varnothing$ and consequently $U \cap \tau_{1} \operatorname{cl} A \subset A$ so that $U \cap \tau_{1} \operatorname{cl} A \subset U \cap A$. Thus $U \cap \tau_{1} \operatorname{cl} A=U \cap A$, i.e., the set $A$ is $(2,1)$-locally closed at the point $x$.

Conversely, let $A$ be a $(2,1)$-locally closed set at a point $x \in A$. Then there is a set $U \in \tau_{2}$ such that $x \in U$ and $U \cap A=U \cap \tau_{1}$ cl $A$. Moreover, $U \in \tau_{2}$ implies $U \cap \tau_{2} \operatorname{cl}(U \cap A)=U \cap \tau_{2} \operatorname{cl} A \subset U \cap \tau_{1} \operatorname{cl} A=U \cap A$. Hence $\tau_{1} \operatorname{cl} A \backslash A \subset$ $\tau_{1} \operatorname{cl} A \backslash(U \cap A) \subset \tau_{1} \operatorname{cl} A \backslash\left(U \cap \tau_{2} \operatorname{cl}(U \cap A)\right)=\left(\tau_{1} \operatorname{cl} A \backslash U\right) \cup\left(\tau_{1} \operatorname{cl} A \backslash \tau_{2} \operatorname{cl}(U \cap A)\right)$, i.e., $\tau_{1} \operatorname{cl} A \backslash A \subset\left(\tau_{1} \operatorname{cl} A \backslash U\right) \cup\left(\tau_{1} \operatorname{cl} A \backslash \tau_{2} \operatorname{cl}(U \cap A)\right)$.

Since $\left(\tau_{1} \operatorname{cl} A \backslash \tau_{2} \operatorname{cl}(U \cap A)\right) \cap U=\left(\tau_{1} \operatorname{cl} A \cap U\right) \backslash\left(\tau_{2} \operatorname{cl}(U \cap A) \cap U\right)=(A \cap$ $U) \backslash\left(\tau_{2} \operatorname{cl} A \cap U\right)=\varnothing$, we have $\tau_{1} \operatorname{cl} A \backslash \tau_{2} \operatorname{cl}(U \cap A) \subset X \backslash U$. Clearly, $\tau_{1} \operatorname{cl} A \backslash U \subset$ $X \backslash U$. Thus by inclusion (1), $\tau_{1} \operatorname{cl} A \backslash A \subset X \backslash U$ and therefore $\tau_{2} \operatorname{cl}\left(\tau_{1} c l A \backslash A\right) \subset$ $X \backslash U$ because $U \in \tau_{2}$. But $x \in U$ and hence $x \bar{\epsilon} A \cap \tau_{2} \operatorname{cl}\left(\tau_{1} \operatorname{cl} A \backslash A\right)$.

Proposition 2.2. Let $A$ be a subset of a $\mathrm{BS}\left(X, \tau_{1}, \tau_{2}\right)$. Then the conditions below are equivalent:

(1) $A \in(i, j)-\mathcal{L C}(X)$.

(2) $A \in \tau_{i}^{\prime}$ in a $\operatorname{BsS}\left(\tau_{j} \operatorname{cl} A, \tau_{1}^{\prime}, \tau_{2}^{\prime}\right)$.

(3) $A=U \cap F$, where $U \in \tau_{i}$ and $F \in \operatorname{co} \tau_{j}$.

Proof. $\quad(1) \Longrightarrow(2)$. If $A \in(i, j)-\mathcal{L C}(X)$, then for every point $x \in A$ there is an $i$-open neighborhood $U(x)$ such that $U(x) \cap A=U(x) \cap \tau_{j}$ cl $A$. But $A=\cup_{x \in A}(U(x) \cap A)=\cup_{x \in A}\left(U(x) \cap \tau_{j} \operatorname{cl} A\right)$ and for every point $x \in A$ the set $U(x) \cap \tau_{j} \mathrm{cl} A$ is $i$-open in $\left(\tau_{j} \mathrm{cl} A, \tau_{1}^{\prime}, \tau_{2}^{\prime}\right)$. Therefore $A$ is $i$-open in $\left(\tau_{j} \operatorname{cl} A, \tau_{1}^{\prime}, \tau_{2}^{\prime}\right)$. 
$(2) \Longrightarrow(1)$. Let $x \in A$ be any point. Then there is an $i$-open set $U$ such that $A=U \cap \tau_{j} \operatorname{cl} A$. Hence $x \in U$ and $U \cap A=U \cap \tau_{j} \operatorname{cl} A$.

$(2) \Longleftrightarrow(3)$ is obvious.

Corollary. In a $\mathrm{BS}\left(X, \tau_{1}, \tau_{2}\right)$ we have $\tau_{i} \cup \operatorname{co} \tau_{j} \subset(i, j)$ - $\mathcal{L C}(X)$. Moreover, in a $\mathrm{BS}\left(X, \tau_{1}<\tau_{2}\right)$ for every subset $A \subset X$ we have the equivalence

$$
A \in(2,1)-\mathcal{L C}(X) \Longleftrightarrow \tau_{2} \operatorname{cl}\left(\tau_{1} \operatorname{cl} A \backslash A\right)=\tau_{1} \operatorname{cl} A \backslash A
$$

and the inclusions

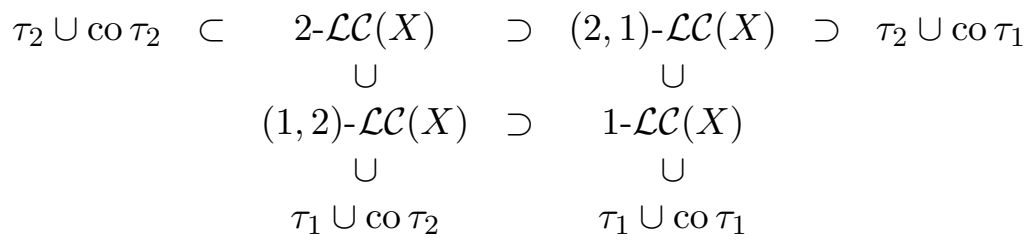

Proof. Indeed, the first inclusion is correct by (3) of Proposition 2.2, while the others are immediate consequences of the inclusions given after Definition 2.3 .

If $\tau_{1} \operatorname{cl} A \backslash A=\tau_{2} \operatorname{cl}\left(\tau_{1} \operatorname{cl} A \backslash A\right)$, then the equality $A=\tau_{1} \operatorname{cl} A \backslash\left(\tau_{1} \operatorname{cl} A \backslash A\right)$ implies that $A$ is a difference of the 1-closed set and the 2-closed set and therefore $A$ is the meet of the 1-closed set with the 2-open set.

On the other hand, if $A=F \cap U$, where $F \in \operatorname{co} \tau_{1}$ and $U \in \tau_{2}$, then $A=F \backslash B$, where $B=X \backslash U \in \cos \tau_{2}$. Hence $A=\tau_{1} \operatorname{cl} A \cap(F \backslash B)=\left(\tau_{1} \operatorname{cl} A \cap\right.$ $F) \backslash\left(\tau_{1} \operatorname{cl} A \cap B\right)$. From $A \subset F$ and $F \in \operatorname{co} \tau_{1}$ we obtain $\tau_{1} \operatorname{cl} A \subset F$ and therefore $\tau_{1} \operatorname{cl} A \cap F=\tau_{1} \operatorname{cl} A$. Thus $A=\tau_{1} \operatorname{cl} A \backslash\left(\tau_{1} \operatorname{cl} A \cap B\right)$ and $\tau_{1} \operatorname{cl} A \backslash A=$ $\tau_{1} \operatorname{cl} A \cap B \in \operatorname{co} \tau_{2}$ as $\tau_{1} \subset \tau_{2}$.

Definition 2.4. $A$ BS $\left(X, \tau_{1}, \tau_{2}\right)$ is $(i, j)$-regular if for each point $x \in X$ and each $i$-closed set $F \subset X, x \bar{\epsilon} F$, there exist an $i$-open set $U \subset X$ and $a$ $j$-open set $V \subset X$ such that $x \in U, F \subset V$ and $U \cap V=\varnothing$ [15].

It is not difficult to see that $\left(X, \tau_{1}, \tau_{2}\right)$ is $(i, j)$-regular $\Longleftrightarrow$ for each point $x \in X$ and each $i$-open set $U \subset X, x \in U$, there exists an $i$-open set $V$ such that $x \in V \subset \tau_{j} \operatorname{cl} V \subset U$.

Theorem 2.3. A subset $A$ of an (i,j)-regular BS $\left(X, \tau_{1}, \tau_{2}\right)$ is $(i, j)$ locally closed at a point $x \in A$ if and only if there exists an $i$-neighborhood $U(x)$ such that $U(x) \cap A=\tau_{j} \operatorname{cl}(U(x) \cap A)$.

Proof. If $A \subset X$ is $(i, j)$-locally closed at a point $x \in A$, then there is a set $U \in \tau_{i}$ such that $x \in U$ and $U \cap A=U \cap \tau_{j}$ cl $A$. Hence $U \cap A \subset$ $\tau_{j} \operatorname{cl}\left(U \cap \tau_{j} \operatorname{cl} A\right)$ so that $U \cap A \subset U \cap \tau_{j} \operatorname{cl}\left(U \cap \tau_{j} \operatorname{cl} A\right)$. On the other hand, $\tau_{j} \operatorname{cl}\left(U \cap \tau_{j} \operatorname{cl} A\right) \subset \tau_{j} \operatorname{cl} A$ implies $U \cap \tau_{j} \operatorname{cl}\left(U \cap \tau_{j} \operatorname{cl} A\right) \subset U \cap \tau_{j} \operatorname{cl} A=U \cap A$. Thus $U \cap A=U \cap \tau_{j} \operatorname{cl}\left(U \cap \tau_{j} \operatorname{cl} A\right)=U \cap \tau_{j} \operatorname{cl}(U \cap A)$. Since $x \in U$ and $\left(X, \tau_{1}, \tau_{2}\right)$ 
is $(i, j)$-regular, there is a set $V \in \tau_{i}$ such that $x \in V$ and $\tau_{j} \operatorname{cl} V \subset U$, i.e., $\tau_{j} \operatorname{cl} V \cap U=\tau_{j} \operatorname{cl} V$ and $\tau_{j} \operatorname{cl} V \cap A=\tau_{j} \operatorname{cl} V \cap U \cap A=\tau_{j} \operatorname{cl} V \cap U \cap \tau_{j} \operatorname{cl}(U \cap A)=$ $\left(\tau_{j} \operatorname{cl} V \cap U\right) \cap \tau_{j} \operatorname{cl}(U \cap A)=\tau_{j} \operatorname{cl} V \cap \tau_{j} \operatorname{cl}(U \cap A)$. If $\tau_{j} \operatorname{cl} V=U(x)$, then $U(x)$ is an $i$-neighborhood of $x$ such that $U(x) \cap A \in \operatorname{co} \tau_{j}$, i.e., $U(x) \cap A=$ $\tau_{j} \operatorname{cl}(U(x) \cap A)$.

Conversely, assume that $x \in A$ and $U(x)$ is its $i$-neighborhood such that $U(x) \cap A=\tau_{j} \operatorname{cl}(U(x) \cap A)$. If $U=\tau_{i}$ int $U(x)$, then $U \neq \varnothing$ and $U \cap A=$ $U \cap(U(x) \cap A)=U \cap \tau_{j} \operatorname{cl}(U(x) \cap A)$ so that $U \cap A$ is $j$-closed in $U$. Hence $U \cap A=U \cap \tau_{j}$ cl $A$, i.e., $A$ is $(i, j)$-locally closed at the point $x$.

Note that the requirement that a $\mathrm{BS}\left(X, \tau_{1}, \tau_{2}\right)$ in Theorem 2.3 be $(i, j)$ regular is essential. Indeed, if $\left(X, \tau_{1}, \tau_{2}\right)$ is not $(i, j)$-regular, then there are a point $x \in X$ and a neighborhood $U(x) \in \tau_{i}$ such that $\tau_{j} \operatorname{cl} V(x) \cap(X \backslash U(x)) \neq$ $\varnothing$ for every $i$-open neighborhood $V(x)$. From Corollary of Proposition 2.2 it follows that $U(x) \in(i, j)-\mathcal{L C}(X)$ and thus $U(x) \in(i, j)-\mathcal{L C}(X, x)$. If the condition of Theorem 2.3 is fulfilled, then there exists an $i$-neighborhood $W(x)$ such that $W(x) \cap U(x)=\tau_{j} \operatorname{cl}(W(x) \cap U(x))$. Let $H=\tau_{i}$ int $W(x)$. Then $H \cap U(x)=E(x) \in \tau_{i}$ and $\tau_{j} \operatorname{cl} E(x)=\tau_{j} \operatorname{cl}(H \cap U(x))=\tau_{j} \operatorname{cl}\left(\tau_{i} \operatorname{int} W(x) \cap\right.$ $U(x)) \subset \tau_{j} \mathrm{cl}(W(x) \cap U(x)) \subset U(x)$, which contradicts the assumption.

Definition 2.5. In a $\mathrm{BS}\left(X, \tau_{1}, \tau_{2}\right)$ an $(i, j)$-boundary is an operator $(i, j)$-Fr : $2^{X} \rightarrow p-\operatorname{Cl}(X)$ defined as follows: $(i, j)$-Fr $A=\tau_{i} \operatorname{cl} A \cap \tau_{j} \operatorname{cl}(X \backslash A)$ for each set $A \in 2^{X} \quad[6]$.

The most important properties of this operator are listed in Theorem 1.3.1 [11].

Definition 2.6. A subset $A$ of a $\mathrm{BS}\left(X, \tau_{1}, \tau_{2}\right)$ is an $(i, j)$-open domain in $X$ if $A=\tau_{i} \operatorname{int} \tau_{j} \mathrm{cl} A$. The complement in $X$ of an $(i, j)$-open domain is an $(i, j)$-closed domain in $X$, i.e., a subset $B$ of $X$ is an $(i, j)$-closed domain in $X$ if $B=\tau_{i} \mathrm{cl} \tau_{j}$ int $B$ [22].

The family of all $(i, j)$-open domains $((i, j)$-closed domains) of $X$ is denoted by $(i, j)-\mathcal{O D}(X)((i, j)-\mathcal{C D}(X))$.

Note that the $i$-interior ( $i$-closure) of every intersection (union) of $(i, j)$ open domains $\left((i, j)\right.$-closed domains) of a BS $\left(X, \tau_{1}, \tau_{2}\right)$ is an $(i, j)$-open domain (an $(i, j)$-closed domain) [23].

Theorem 2.4. The following statements are satisfied in a $\mathrm{BS}\left(X, \tau_{1}, \tau_{2}\right)$ : $A \in(i, j)-\mathcal{O D}(X) \Longleftrightarrow A \in \tau_{i}$ and

$(j, i)-\operatorname{Fr} A=(j, i)-\operatorname{Fr} \tau_{j} \operatorname{cl} A=(i, j)-\operatorname{Fr} \tau_{j} \operatorname{int}(X \backslash A) \subset \tau_{i} \operatorname{cl} \tau_{j} \operatorname{int}(X \backslash A)$ so that

$A \in(i, j)-\mathcal{C D}(X) \Longleftrightarrow A \in \cos \tau_{i}$ and

$(i, j)-\operatorname{Fr} A=(i, j)-\operatorname{Fr} \tau_{j} \operatorname{int} A=(j, i)-\operatorname{Fr} \tau_{j} \operatorname{cl}(X \backslash A) \subset \tau_{i} \operatorname{cl} \tau_{j} \operatorname{int} A$. 
Proof. $A=\tau_{i} \operatorname{int} \tau_{j} \operatorname{cl} A$ clearly implies $(j, i)-\operatorname{Fr} \tau_{j} \operatorname{cl} A=\tau_{j} \operatorname{cl} A \cap$ $\tau_{i} \operatorname{cl}\left(X \backslash \tau_{j} \operatorname{cl} A\right)=\tau_{j} \operatorname{cl} A \cap(X \backslash A)=\tau_{j} \operatorname{cl} A \cap \tau_{i} \operatorname{cl}(X \backslash A)=(j, i)$-Fr $A$ and by $(4)$ of Theorem 2.3.1 in [11] we also have $(j, i)-\operatorname{Fr} \tau_{j} \operatorname{cl} A=(i, j)-\operatorname{Fr} \tau_{j} \operatorname{int}(X \backslash A)$. Furthermore, if $A=\tau_{i}$ int $\tau_{j} \mathrm{cl} A$, then $A \in \tau_{i}$ and $(j, i)-\operatorname{Fr} A=\tau_{j} \operatorname{cl} A \cap$ $\tau_{i} \operatorname{cl}(X \backslash A) \subset \tau_{i} \operatorname{cl}(X \backslash A)=\tau_{i} \operatorname{cl} \tau_{j} \operatorname{int}(X \backslash A)$.

Conversely, if $(j, i)$-Fr $A \subset \tau_{i} \operatorname{cl} \tau_{j} \operatorname{int}(X \backslash A)$, then $(j, i)$-Fr $A \cup \tau_{i} \operatorname{cl} \tau_{j}$ $\operatorname{int}(X \backslash A)=\tau_{i} \operatorname{cl} \tau_{j} \operatorname{int}(X \backslash A)$. On the other hand, $(j, i)-\operatorname{Fr} A \cup \tau_{i} \operatorname{cl} \tau_{j} \operatorname{int}(X \backslash A)=$ $\left(\tau_{j} \operatorname{cl} A \cap \tau_{i} \operatorname{cl}(X \backslash A)\right) \cup \tau_{i} \operatorname{cl} \tau_{j} \operatorname{int}(X \backslash A)=X \cap \tau_{i} \operatorname{cl}(X \backslash A)=\tau_{i} \operatorname{cl}(X \backslash A)$. Hence $\tau_{i} \operatorname{cl}(X \backslash A)=\tau_{i} \operatorname{cl} \tau_{j} \operatorname{int}(X \backslash A)$ and $A \in \tau_{i}$ implies $A=\tau_{i} \operatorname{int} A=\tau_{i} \operatorname{int} \tau_{j} \operatorname{cl} A$, i.e, $A \in(i, j)-\mathcal{O D}(X)$.

For the second equivalence it suffices to replace the set $A \in(i, j)-\mathcal{C D}(X)$ by the set $X \backslash A \in(i, j)-\mathcal{O D}(X)$ and to apply (4) of Theorem 1.3.1 in [11].

Corollary. The following conditions are satisfied in a $\mathrm{BS}\left(X, \tau_{1}<\tau_{2}\right)$ :

(1) $A \in \tau_{1} \cap(2,1)-\mathcal{O D}(X) \Longrightarrow(1,2)-\operatorname{Fr} A=1-\operatorname{Fr} A$ and $A \in \tau_{1} \cap$ $2-\mathcal{O D}(X) \Longrightarrow 2-\operatorname{Fr} A=(2,1)-\operatorname{Fr} A$.

(2) $1-\mathcal{O D}(X) \subset(1,2)-\mathcal{O D}(X),(2,1)-\mathcal{O D}(X) \subset 2-\mathcal{O D}(X)$ and $\tau_{1} \cap(2,1)$ $\mathcal{O D}(X)=(1,2)-\mathcal{O D}(X) \cap(2,1)-\mathcal{O D}(X)=1-\mathcal{O D}(X) \cap(2,1)-\mathcal{O D}(X)$ $\subset 1-\mathcal{O D}(X) \cap 2-\mathcal{O D}(X) \subset \tau_{1} \cap 2-\mathcal{O D}(X)=(1,2)-\mathcal{O D}(X) \cap 2-\mathcal{O D}(X)$ so that $1-\mathcal{C D}(X) \subset(1,2)-\mathcal{C D}(X),(2,1)-\mathcal{C D}(X) \subset 2-\mathcal{C D}(X)$ and $\operatorname{co} \tau_{1} \cap(2,1)-\mathcal{C D}(X)=(1,2)-\mathcal{C D}(X) \cap(2,1)-\mathcal{C D}(X)=1-\mathcal{C D}(X) \cap$ $(2,1)-\mathcal{C D}(X) \subset 1-\mathcal{C D}(X) \cap 2-\mathcal{C D}(X) \subset \operatorname{co} \tau_{1} \cap 2-\mathcal{C D}(X)=(1,2)-\mathcal{C D}(X) \cap$ $2-\mathcal{C D}(X)$.

Proof. (1) By virtue of Theorem 2.4, $A \in \tau_{1} \cap(2,1)-\mathcal{O D}(X) \Longrightarrow$ $(1,2)-\operatorname{Fr} A=(1,2)-\operatorname{Fr} \tau_{1} \operatorname{cl} A=\tau_{1} \operatorname{cl} A \cap \tau_{2} \operatorname{cl}\left(X \backslash \tau_{1} A\right)=\tau_{1} \operatorname{cl} A \cap(X \backslash A)=$ $\tau_{1} \operatorname{cl} A \cap \tau_{1} \operatorname{cl}(X \backslash A)=1-\operatorname{Fr} A ; A \in \tau_{1} \cap 2-\mathcal{O D}(X) \Longrightarrow 2 \operatorname{Fr} A=2-\operatorname{Fr} \tau_{2} \operatorname{cl} A=$ $\tau_{2} \operatorname{cl} A \cap \tau_{2} \operatorname{cl}\left(X \backslash \tau_{2} A\right)=\tau_{2} \operatorname{cl} A \cap(X \backslash A)=\tau_{2} \operatorname{cl} A \cap \tau_{1} \operatorname{cl}(X \backslash A)=(2,1)-\mathrm{Fr} A$.

(2) $A \in 1-\mathcal{O D}(X) \Longrightarrow A \in \tau_{1}$ and 1-Fr $A \subset \tau_{1} \operatorname{cl} \tau_{1} \operatorname{int}(X \backslash A) \Longrightarrow A \in \tau_{1}$ and (2,1)-Fr $A \subset 1-\operatorname{Fr} A \subset \tau_{1} \operatorname{cl} \tau_{1} \operatorname{int}(X \backslash A) \subset \tau_{1} \operatorname{cl} \tau_{2} \operatorname{int}(X \backslash A) \Longrightarrow A \in$ $(1,2)-\mathcal{O D}(X)$.

$A \in(2,1)-\mathcal{O D}(X) \Longrightarrow A \in \tau_{2}$ and $(1,2)-\operatorname{Fr} A \subset \tau_{2} \operatorname{cl} \tau_{1} \operatorname{int}(X \backslash A) \Longrightarrow$ $A \in \tau_{2}$ and 2-Fr $A \subset(1,2)-\operatorname{Fr} A \subset \tau_{2} \operatorname{cl} \tau_{1} \operatorname{int}(X \backslash A) \subset \tau_{2} \operatorname{cl} \tau_{2} \operatorname{int}(X \backslash A) \Longrightarrow$ $A \in 2-\mathcal{O D}(X)$.

Further, by (1), $A \in \tau_{1} \cap(2,1)-\mathcal{O D}(X) \Longrightarrow A \in \tau_{2}$ and 1-Fr $A=$ $(1,2)-\operatorname{Fr} A \subset \tau_{2} \operatorname{cl} \tau_{1} \operatorname{int}(X \backslash A) \subset \tau_{1} \operatorname{cl} \tau_{1} \operatorname{int}(X \backslash A) \Longrightarrow A \in 1-\mathcal{O D}(X)$. Similarly, $A \in \tau_{1} \cap 2-\mathcal{O D}(X) \Longrightarrow A \in \tau_{2}$ and $(2,1)$-Fr $A=2-\operatorname{Fr} A \subset \tau_{2} \operatorname{cl} \tau_{2}$ $\operatorname{int}(X \backslash A) \subset \tau_{1} \operatorname{cl} \tau_{2} \operatorname{int}(X \backslash A) \Longrightarrow A \in(1,2)-\mathcal{O D}(X)$. Hence, taking into account the inclusion $1-\mathcal{O D}(X) \subset(1,2)-\mathcal{O D}(X) \subset \tau_{1}$, we find that $\tau_{1} \cap$ $(2,1)-\mathcal{O D}(X)=(1,2)-\mathcal{O D}(X) \cap(2,1)-\mathcal{O D}(X)=1-\mathcal{O D}(X) \cap(2,1)-\mathcal{O D}(X)$ and $\tau_{1} \cap 2-\mathcal{O D}(X)=(1,2)-\mathcal{O D}(X) \cap 2-\mathcal{O D}(X)$. The rest is obvious. 
Finally, note that such a simple argument as $\tau_{1} \subset \tau_{2}$ is helpful in establishing in a different way the inclusions $1-\mathcal{O D}(X) \subset(1,2)-\mathcal{O D}(X)$ and $(2,1)-\mathcal{O D}(X) \subset 2-\mathcal{O D}(X)$. Indeed, $A \in 1-\mathcal{O D}(X) \Longrightarrow A=\tau_{1}$ int $\tau_{1} \operatorname{cl} A \Longrightarrow$ $A=\tau_{1} \operatorname{int} A \Longrightarrow A \subset \tau_{1} \operatorname{int} \tau_{2} \operatorname{cl} A \subset \tau_{1} \operatorname{int} \tau_{1} \operatorname{cl} A=A$, i.e, $A=\tau_{1} \operatorname{int} \tau_{2} \operatorname{cl} A$; $A \in(2,1)-\mathcal{O D}(X) \Longrightarrow A=\tau_{2} \operatorname{int} \tau_{1} \operatorname{cl} A \Longrightarrow A \subset \tau_{2} \operatorname{int} \tau_{2} \operatorname{cl} A \subset \tau_{2} \operatorname{int} \tau_{1}$ $\operatorname{cl} A=A$, i.e, $A \in 2-\mathcal{O D}(X)$.

The inverse inclusions in the foregoing corollary are not, generally speaking, correct in a $\mathrm{BS}\left(X, \tau_{1}<\tau_{2}\right)$.

Example 2.1. Let $X=\{a, b, c, d\}, \tau_{1}=\{\varnothing,\{a\},\{c\},\{a, c\}, X\}, \tau_{2}=$ $\{\varnothing,\{a\},\{c\},\{a, c\},\{a, b\},\{a, b, c\}, X\}$. Then $\{a\} \in 1-\mathcal{O D}(X) \subset(1,2)-\mathcal{O D}(X)$, $\{a\} \bar{\in} 2-\mathcal{O D}(X)$ so that $\{a\} \bar{\in}(2,1)-\mathcal{O D}(X)$. Also, $\{a, b\} \in(2,1)-\mathcal{O D}(X) \subset$ $2-\mathcal{O D}(X)$, but $\{a, b\} \bar{\in}(1,2)-\mathcal{O D}(X)$ and therefore $\{a, b\} \bar{\in} 1-\mathcal{O D}(X)$.

Example 2.2. Let $X$ be as in Example 2.1, $\tau_{1}=\{\varnothing,\{a\},\{a, b, c\}, X\}$, $\tau_{2}=\{\varnothing,\{a\},\{d\},\{a, d\},\{b, c\},\{a, b, c\},\{b, c, d\}, X\}$. Then $\{a\} \in(1,2)$ $\mathcal{O D}(X) \backslash 1-\mathcal{O D}(X),\{a, d\} \in 2-\mathcal{O D}(X) \backslash(2,1)-\mathcal{O D}(X)$.

Proposition 2.3. In a $\mathrm{BS}\left(X, \tau_{1}<\tau_{2}\right)$ we have $U \in \tau_{2} \Longleftrightarrow U=$ $V \backslash \tau_{2} \operatorname{cl} A$, where $V \in(2,1)-\mathcal{O D}(X)$ and $A \in(1,2)-\mathcal{N D}(X)$ so that $F \in$ co $\tau_{2} \Longleftrightarrow F=B \cup \tau_{2} \operatorname{cl} A$, where $B \in(2,1)-\mathcal{C D}(X)$ and $A \in(1,2)-\mathcal{N D}(X)$.

Proof. Let $U \in \tau_{2}$ and $V=\tau_{2}$ int $\tau_{1} \operatorname{cl} U$. By (1) of Proposition 2.3.1 in [11] the difference $\tau_{1} \operatorname{cl} U \backslash U \in \operatorname{co} \tau_{2} \cap 1-\mathcal{B} d(X) \subset(1,2)-\mathcal{N D}(X)$ and hence $A=$ $V \backslash U \in(1,2)-\mathcal{N D}(X)$. Clearly, $U=V \backslash A$ and $\tau_{2} \operatorname{cl} A=\tau_{2} \operatorname{cl}(V \backslash U) \subset \tau_{2} \operatorname{cl} V \cap$ $\tau_{2} \operatorname{cl}(X \backslash U)=\tau_{2} \operatorname{cl} V \backslash U$. Thus $U=V \cap U=V \backslash\left(\tau_{2} \operatorname{cl} V \backslash U\right) \subset V \backslash \tau_{2} \operatorname{cl} A \subset$ $V \backslash A=U$, i.e., $U=V \backslash \tau_{2} \operatorname{cl} A$.

The converse implication is obvious.

If $F \in \operatorname{co} \tau_{2}$, then $X \backslash F=V \backslash \tau_{2} \operatorname{cl} A$, where $V \in(2,1)-\mathcal{O D}(X)$ and $A \in(1,2)-\mathcal{N D}(X)$. Hence $F=(X \backslash V) \cup \tau_{2} \operatorname{cl} A=B \cup \tau_{2} \operatorname{cl} A$ with $B \in$ $(2,1)-\mathcal{C D}(X)$.

Below we will define and study important modifications of the notions of a set dense in itself, a perfect set and a scattered set.

Definition 2.7. A subset $A$ of a $\mathrm{BS}\left(X, \tau_{1}, \tau_{2}\right)$ is $(i, j)$-dense in itself if $A_{i}^{i} \subset A_{j}^{d}[9]$.

The family of all subsets of $X,(i, j)$-dense in themselves, ( $i$-discrete) is denoted by $(i, j)-\mathcal{D} \mathcal{I}(X)(i-\mathcal{I}(\mathcal{X}))$.

Theorem 2.5. Let $\left(X, \tau_{1}, \tau_{2}\right)$ be a BS. Then

(1) 1 -DI $\mathcal{I}(X) \cup 2-\mathcal{D} \mathcal{I}(X) \subset(1,2)-\mathcal{D I}(X)=(2,1)-\mathcal{D I}(X)$ and in a $\mathrm{BS}\left(X, \tau_{1}<\tau_{2}\right)$ we also have 
(2) $2-\mathcal{D} \mathcal{I}(X) \subset 1-\mathcal{D} \mathcal{I}(X)=(1,2)-\mathcal{D} \mathcal{I}(X)=(2,1)-\mathcal{D I}(X), 1-\mathcal{I}(X) \subset 2-\mathcal{I}(X)$.

Proof. It is clear that the inclusion in (1) follows from the equality in (1) and thus it suffices to prove only the equality.

Let $A \in(i, j)-\mathcal{D I}(X)$. Then $A_{i}^{i} \subset A_{j}^{d}$ so that $X \backslash A_{j}^{d} \subset X \backslash A_{i}^{i}$. Hence $A \cap\left(X \backslash A_{j}^{d}\right) \subset A \cap\left(X \backslash A_{i}^{i}\right)$, i.e., $A \backslash A_{j}^{d} \subset A \backslash A_{i}^{i}$ and $A_{j}^{i} \subset A \backslash A_{i}^{i} \subset A_{i}^{d}$. Thus $A \in(j, i)-\mathcal{D} \mathcal{I}(X)$.

(2) $\tau_{1} \subset \tau_{2}$ implies that $A_{2}^{d} \subset A_{1}^{d}$ and $A_{1}^{i} \subset A_{2}^{i}$ for every subset $A \subset X$, i.e., $2-\mathcal{D I}(X) \subset 1-\mathcal{D} \mathcal{I}(X)$ and $1-\mathcal{I}(X) \subset 2-\mathcal{I}(X)$. By Definition 2.7, $A \in$ $(1,2)-\mathcal{D I}(X) \Longleftrightarrow A_{1}^{i} \subset A_{2}^{d}$. Hence $A_{1}^{i} \subset A_{1}^{d} \Longrightarrow A_{1}^{i}=\varnothing \Longrightarrow A \subset A_{1}^{d} \Longleftrightarrow$ $A \in 1-\mathcal{D I}(X)$.

From this theorem we conclude that if in a BS $\left(X, \tau_{1}<\tau_{2}\right)$ a set $A \subset$ $X$ has at least one 1-isolated point, then $A \bar{\in} 1-\mathcal{D} \mathcal{I}(X)=(1,2)-\mathcal{D I}(X)=$ $(2,1)-\mathcal{D} \mathcal{I}(X)$.

By the first equality in (1) of Theorem 2.5 it suffices to consider only the family $(1,2)-\mathcal{D I}(X)$.

Example 2.3. Suppose we are given the BS $\left(\mathbb{R}, \tau_{1}, \tau_{2}\right)$, where $\tau_{1}=$ $\omega$ is the natural topology on $\mathbb{R}, \tau_{2}$ is the discrete topology on $\mathbb{R}$ and $A=$ $\left\{1, \frac{1}{2}, \frac{1}{3}, \frac{1}{4} \ldots\right\} \subset \mathbb{R}$. Then $A_{1}^{d}=\{0\}$ and therefore $A_{1}^{i}=A \backslash A_{1}^{d}=A, A_{2}^{i}=A$ and $A_{2}^{d}=\varnothing$. Hence $A \bar{\epsilon}(1,2)-\mathcal{D} \mathcal{I}(\mathbb{R})$.

The converse of the first inclusion in Theorem 2.5 does hold in general.

Example 2.4. Suppose $\tau_{1}$ is the antidiscrete topology on $\mathbb{R}, \tau_{2}=\omega$ is the natural topology on $\mathbb{R}$ and a subset $A \subset \mathbb{R}$ is the same as in Example 2.3. Then $A_{1}^{i}=\varnothing, A_{1}^{d}=\mathbb{R}, A_{2}^{i}=A$ and $A_{2}^{d}=\{0\}$. Hence $A_{1}^{i}=\varnothing$ implies that $A \in 1-\mathcal{D I}(\mathbb{R})=(1,2)-\mathcal{D I}(\mathbb{R})$, but $A \bar{\in} 2-\mathcal{D I}(\mathbb{R})$ since $A_{2}^{i} \neq \varnothing$.

Theorem 2.6. In a $\mathrm{BS}\left(X, \tau_{1}, \tau_{2}\right)$ any union of sets $(1,2)$-dense in themselves is $(1,2)$-dense in itself.

Proof. Let $\left\{A_{s}\right\}_{s \in S}$ be any family of sets $(1,2)$-dense in themselves so that $\left(A_{s}\right)_{1}^{i}=\left(A_{s} \backslash\left(A_{s}\right)_{1}^{d}\right) \subset\left(A_{s}\right)_{2}^{d}$ for every $s \in S$. It is well known that $\cup_{s \in S}\left(A_{s}\right)_{1}^{d} \subset\left(\cup_{s \in S} A_{s}\right)_{1}^{d}$ and hence $\left(\cup_{s \in S} A_{s}\right) \backslash\left(\cup_{s \in S} A_{s}\right)_{1}^{d} \subset \cup_{s \in S} A_{s} \backslash \cup_{s \in S}\left(A_{s}\right)_{1}^{d} \subset$ $\cup_{s \in S}\left(A_{s} \backslash\left(A_{s}\right)_{1}^{d}\right) \subset \cup_{s \in S}\left(A_{s}\right)_{2}^{d} \subset\left(\cup_{s \in S} A_{s}\right)_{2}^{d}$.

If there exists a set $A \in i \mathcal{D}(X) \cap i-\mathcal{B} d(X)$, then by virtue of Theorem 4 from [17, p. 83], $X \in i$-DII $(X) \subset(1,2)-\mathcal{D I}(X)$. Based on this fact, our next theorem gives examples of new members of the family $(1,2)-\mathcal{D I}(X)$.

Theorem 2.7. For every subset $A$ of a $\mathrm{BS}\left(X, \tau_{1}<\tau_{2}\right)$ we have $\tau_{1} \operatorname{int}(1,2)-\operatorname{Fr} A, A \cap \tau_{1} \operatorname{int}(1,2)-\operatorname{Fr} A \in(1,2)-\mathcal{D} \mathcal{I}(X)$. 
Proof. As is well-known, if $U \in \tau_{1}$ and $A \subset X$ is any set, then $\tau_{1} \operatorname{cl}(U \cap$ $\left.\tau_{1} \operatorname{cl} A\right)=\tau_{1} \operatorname{cl}(U \cap A)$. Therefore $\tau_{1} \operatorname{cl}(U \cap A)=\tau_{1} \operatorname{cl}\left(U \cap \tau_{2} \operatorname{cl} A\right)$.

Let $U=\tau_{1} \operatorname{int}(1,2)-\operatorname{Fr} A=\tau_{1} \operatorname{int}(2,1)-\operatorname{Fr}(X \backslash A)$. Then it is obvious that $\tau_{1} \operatorname{cl} U=\tau_{1} \operatorname{cl}\left(U \cap \tau_{1} \operatorname{cl} A\right)=\tau_{1} \operatorname{cl}(U \cap A)$ and $\tau_{1} \operatorname{cl} U=\tau_{1} \operatorname{cl}\left(U \cap \tau_{2} \operatorname{cl}(X \backslash A)\right)=$ $\tau_{1} \operatorname{cl}(U \cap(X \backslash A))=\tau_{1} \operatorname{cl}(U \backslash A)$, i.e.,

$$
\begin{gathered}
\tau_{1} \operatorname{cl} \tau_{1} \operatorname{int}(1,2)-\operatorname{Fr} A= \\
=\tau_{1} \operatorname{cl}\left(A \cap \tau_{1} \operatorname{int}(1,2)-\operatorname{Fr} A\right)=\tau_{1} \operatorname{cl}\left(\tau_{1} \operatorname{int}(1,2)-\operatorname{Fr} A \backslash A\right) .
\end{gathered}
$$

The first equality in (1) shows that the set $A \cap \tau_{1} \operatorname{int}(1,2)-\operatorname{Fr} A$ is 1 dense in $\tau_{1} \operatorname{int}(1,2)$-Fr $A$. Moreover, (1) also implies that $\tau_{1} \operatorname{int}(1,2)-\operatorname{Fr} A \subset$ $\tau_{1} \operatorname{cl}\left(\tau_{1} \operatorname{int}(1,2)-\operatorname{Fr} A \backslash A\right) \subset \tau_{1} \operatorname{cl}\left(\tau_{1} \operatorname{int}(1,2)-\operatorname{Fr} A \backslash\left(A \cap \tau_{1} \operatorname{int}(1,2)-\operatorname{Fr} A\right)\right)$ so that $A \cap \tau_{1} \operatorname{int}(1,2)-\operatorname{Fr} A$ is the 1-boundary in $\tau_{1} \operatorname{int}(1,2) \operatorname{Fr} A[17$, p. 76]. Hence by virtue of the remark preceding this theorem and Theorem 2.5 we obtain $\tau_{1} \operatorname{int}(1,2)-\operatorname{Fr} A \in 1-\mathcal{D I}(X)=(1,2)-\mathcal{D I}(X)$. Since $A \cap \tau_{1} \operatorname{int}(1,2)-\operatorname{Fr} A$ is 1 dense in $\tau_{1} \operatorname{int}(1,2)$-Fr $A$, where $\tau_{1} \operatorname{int}(1,2)$-Fr $A \in 1-\mathcal{D} \mathcal{I}(X)=(1,2)-\mathcal{D I}(X)$, by virtue of Theorem 3 from [17, p. 83] we obtain $A \cap \tau_{1} \operatorname{int}(1,2)-\operatorname{Fr} A \in$ $1-\mathcal{D} \mathcal{I}(X)=(1,2)-\mathcal{D} \mathcal{I}(X)$.

Definition 2.8. A subset $A$ of a $\mathrm{BS}\left(X, \tau_{1}, \tau_{2}\right)$ is $(i, j)$-perfect if $A_{j}^{i} \subset$ $A_{i}^{d} \subset A[9]$.

The family of all $(i, j)$-perfect subsets of $X$ is denoted by $(i, j)-\mathcal{P}(X)$. Hence $(i, j) \mathcal{- P}(X)=\operatorname{co} \tau_{i} \cap(1,2)-\mathcal{D} \mathcal{I}(X)$.

Theorem 2.8. In a $\mathrm{BS}\left(X, \tau_{1}, \tau_{2}\right)$ we have $i \mathcal{P}(X) \subset(i, j) \mathcal{P}(X)$ and in $a \operatorname{BS}\left(X, \tau_{1}<\tau_{2}\right)$ we have $(1,2) \mathcal{P}(X)=1 \mathcal{P}(X) \subset(2,1) \mathcal{P}(X) \supset 2 \mathcal{P}(X) \subset$ $1-\mathcal{D I}(X)$.

Proof. By (1) of Theorem 2.5, $i-\mathcal{P}(X)=\operatorname{co} \tau_{i} \cap i-\mathcal{D I}(X) \subset \operatorname{co} \tau_{i} \cap$ $(1,2)-\mathcal{D} \mathcal{I}(X)=(i, j) \mathcal{P}(X)$. Further, if $\tau_{1} \subset \tau_{2}$, then by (2) of Theorem 2.5, $(1,2)-\mathcal{P}(X)=\operatorname{co} \tau_{1} \cap(1,2)-\mathcal{D} \mathcal{I}(X)=\operatorname{co} \tau_{1} \cap 1-\mathcal{D I}(X)=1 \mathcal{P}(X) \subset \operatorname{co} \tau_{2} \cap$ $(1,2)-\mathcal{D I}(X)=(2,1)-\mathcal{P}(X)$. Finally, $2-\mathcal{P}(X)=\operatorname{co} \tau_{2} \cap 2-\mathcal{D} \mathcal{I}(X) \subset 2-\mathcal{D} \mathcal{I}(X) \subset$ $1-\mathcal{D I}(X)$ and $2 \mathcal{P}(X)=\operatorname{co} \tau_{2} \cap 2-\mathcal{D I}(X) \subset \operatorname{co} \tau_{2} \cap(2,1)-\mathcal{D I}(X)=\operatorname{co} \tau_{2} \cap$ $(1,2)-\mathcal{D} \mathcal{I}(X)=(2,1)-\mathcal{P}(X)$.

The inverse inclusions in the latter theorem are not, generally speaking, correct.

Example 2.5. Let $\left(\mathbb{R}, \tau_{1}, \tau_{2}\right)$ be the $\mathrm{BS}$ from the previous example. If $A=\left\{1, \frac{1}{2}, \frac{1}{3}, \frac{1}{4}, \ldots, 0\right\}$, then $A_{1}^{i}=\varnothing, A_{2}^{d}=\{0\}$ and hence $A_{1}^{i} \subset A_{2}^{d} \subset A$ so that $A \in(2,1) \mathcal{P}(\mathbb{R})$. But $A \bar{\in} 2 \mathcal{P}(\mathbb{R})$ since $A \neq A_{2}^{d}$. It is likewise easy to observe that for the set $\mathbb{Z}$ we have $\mathbb{Z}_{1}^{i}=\varnothing, \mathbb{Z}_{1}^{d}=\mathbb{R}, \mathbb{Z}_{2}^{i}=\mathbb{Z}$ and $\mathbb{Z}_{2}^{d}=\varnothing$. Hence $\mathbb{Z} \in(2,1) \mathcal{P}(\mathbb{R})$ but $\mathbb{Z} \bar{\in}(1,2) \mathcal{P}(\mathbb{R})$ since $\mathbb{R}=\mathbb{Z}_{1}^{d}$ is not contained in $\mathbb{Z}$. 
Theorem 2.9. For a $j$ - $\mathrm{T}_{1}$ BS $\left(X, \tau_{1}, \tau_{2}\right)$ we have $j-\mathrm{Cl}(1,2)-\mathcal{D} \mathcal{I}(X) \subset$ $(j, i) \mathcal{P}(X)$.

Proof. We will show that $A_{i}^{i} \subset A_{j}^{d} \Longrightarrow\left(\tau_{j} \operatorname{cl} A\right)_{i}^{i} \subset\left(\tau_{j} \mathrm{cl} A\right)_{j}^{d} \subset \tau_{j} \operatorname{cl} A$.

Clearly, $\left(\tau_{j} \operatorname{cl} A\right)_{j}^{d} \subset \tau_{j} \operatorname{cl}\left(\tau_{j} \operatorname{cl} A\right)=\tau_{j} \operatorname{cl} A$. Further, $\left(\tau_{j} \operatorname{cl} A\right)_{i}^{i}=\tau_{j} \operatorname{cl} A \backslash$ $\left(\tau_{j} \mathrm{cl} A\right)_{i}^{d}=\left(A \cup A_{j}^{d}\right) \backslash\left(A \cup A_{j}^{d}\right)_{i}^{d}=\left(A \cup A_{j}^{d}\right) \backslash\left(A_{i}^{d} \cup\left(A_{j}^{d}\right)_{i}^{d}\right)=\left(\left(A \cup A_{j}^{d}\right) \backslash A_{i}^{d}\right) \cap$ $\left(\left(A \cup A_{j}^{d}\right) \backslash\left(A_{j}^{d}\right)_{i}^{d}\right)=\left(\left(A \backslash A_{i}^{d}\right) \cup\left(A_{j}^{d} \backslash A_{i}^{d}\right)\right) \cap\left(\left(A \backslash\left(A_{j}^{d}\right)_{i}^{d}\right) \cup\left(A_{j}^{d} \backslash\left(A_{j}^{d}\right)_{i}^{d}\right)\right)$. Since $A_{j}^{d} \backslash A_{i}^{d} \subset A_{j}^{d}$ and $A \backslash A_{i}^{d}=A_{i}^{i} \subset A_{j}^{d}$, we conclude that the union in the first brackets above is contained in $A_{j}^{d}$. Hence the set $\left(\tau_{j} \operatorname{cl} A\right)_{i}^{i}$, being the meet, is completely contained in $A_{j}^{d}$. But $A_{j}^{d}=\left(\tau_{j} \mathrm{cl} A\right)_{j}^{d}$ and therefore $\left(\tau_{j} \mathrm{cl} A\right)_{i}^{i} \subset$ $\left(\tau_{j} \operatorname{cl} A\right)_{j}^{d} \subset \tau_{j} \operatorname{cl} A$.

Using the equality $(1,2)-\mathcal{D I}(X)=(2,1)-\mathcal{D I}(X)$, we introduce

Definition 2.9. A subset $A$ of a $\mathrm{BS}\left(X, \tau_{1}, \tau_{2}\right)$ is $(i, j)$-scattered if $A$ is nonempty and contains no nonempty $(i, j)$-dense in itself subset [9].

The family of all $(i, j)$-scattered subsets of $X$ is denoted by $(i, j)-\mathcal{S T}(X)$. It is clear that $(1,2)-\mathcal{D I}(X)=(2,1)-\mathcal{D I}(X) \Longrightarrow(1,2)-\mathcal{S} \mathcal{T}(X)=(2,1)$ $\mathcal{S T}(X)$.

The latter definition and Theorem 2.5 readily yield

Theorem 2.10. The following conditions are satisfied in a $\mathrm{BS}\left(X, \tau_{1}, \tau_{2}\right)$ :

(1) $(1,2)-\mathcal{S T}(X) \subset 1-\mathcal{S T}(X) \cap 2-\mathcal{S T}(X)$.

(2) $A \in(1,2)-\mathcal{S T}(X)$ and $B \subset A, B \neq \varnothing \Longrightarrow B \in(1,2)-\mathcal{S T}(X)$.

In a $\mathrm{BS}\left(X, \tau_{1}<\tau_{2}\right)$ we also have

(3) $1-\mathcal{S T}(X)=(1,2)-\mathcal{S T}(X)$.

Remark 2.1. Let $\left(\mathbb{R}, \tau_{1}, \tau_{2}\right)$ be the BS from Example 2.4, where $A=$ $\left\{1, \frac{1}{2}, \frac{1}{3}, \frac{1}{4}, \ldots\right\} \in 1-\mathcal{D I}(\mathbb{R})=(1,2)-\mathcal{D} \mathcal{I}(\mathbb{R})$. If $\varnothing \neq B \subset A$ is any subset, then $B \in 1-\mathcal{D I}(\mathbb{R})=(1,2)-\mathcal{D I}(\mathbb{R})$ so that $A \bar{\in} 1-\mathcal{S} \mathcal{T}(\mathbb{R})=(1,2)-\mathcal{S} \mathcal{T}(\mathbb{R})$. But $B \subset A \Longrightarrow B_{2}^{d} \subset A_{2}^{d}=\{0\}$ and therefore $B \cap B_{2}^{d}=\varnothing$. Hence $B \bar{\in} 2-\mathcal{D I}(\mathbb{R})$ and, since $B \subset A$ is arbitrary, we obtain $A \in 2-\mathcal{S T}(\mathbb{R})$.

Note further that following Example 2.4 and the above remark we have $A=\left\{1, \frac{1}{2}, \frac{1}{3}, \frac{1}{4}, \ldots\right\} \in 2-\mathcal{I}(\mathbb{R})$ and $A \bar{\epsilon}(1,2)-\mathcal{S} \mathcal{T}(\mathbb{R})$ and thus, generally speaking, the family $i-\mathcal{I}(\mathcal{X})$ is not contained in $(1,2)-\mathcal{S} \mathcal{T}(\mathbb{R})$. However we have

Theorem 2.11. If in a $\mathrm{BS}\left(X, \tau_{1}, \tau_{2}\right)$ a set $A \in i-\mathcal{I}(X)$ and for every subset $B \subset A$ we have $B \bar{\in} j-\mathcal{D I}(X)$, then $A \in(1,2)-\mathcal{S} \mathcal{T}(X)$.

Proof. Assume that $A \in i-\mathcal{I}(X)$ and $B \subset A$ is an arbitrary subset. Then $B \in i-\mathcal{I}(X)$, and $B \bar{\in} j-\mathcal{D I}(X)$ implies that $B_{j}^{i} \neq \varnothing$. Since $B \cap B_{i}^{d}=\varnothing$ and $B_{j}^{i} \subset B$, we obtain $B_{j}^{i} \cap B_{i}^{d}=\varnothing$ so that $B \bar{\in}(1,2)-\mathcal{D I}(X)$. Therefore we have $A \in(1,2)-\mathcal{S T}(X)$ since $B \subset A$ is arbitrary. 
Theorem 2.12. Let $\left(X, \tau_{1}, \tau_{2}\right)$ be a $R-p-\mathrm{T}_{1}\left(\Longleftrightarrow d\right.$ - $\left.\mathrm{T}_{1}\right)$ BS [20]. Then $X=A \cup(X \backslash A)$, where $A \in(1,2)-\mathcal{P}(X) \cap(2,1) \mathcal{P}(X)$ and $X \backslash A \in(1,2)-\mathcal{S} \mathcal{T}(X)$ (it stands to reason that one of these subsets may turn out empty).

Proof. Assume that $A=\cup\{E: E \in(1,2)-\mathcal{D I}(X)\}$. Then by Theorem 2.6, $A \in(1,2)-\mathcal{D} \mathcal{I}(X)$. It follows from Definition 2.8 and Theorem 2.9 that $\tau_{j} \operatorname{cl} A \in(j, i) \mathcal{P}(X) \subset(1,2)-\mathcal{D} \mathcal{I}(X)$. Recalling that $A$ is maximal, we obtain $A \in \operatorname{co} \tau_{1} \cap \operatorname{co} \tau_{2}$ and therefore $A=\tau_{1} \operatorname{cl} A=\tau_{2} \operatorname{cl} A \in(1,2) \mathcal{P}(X) \cap$ $(2,1)-\mathcal{P}(X)$. It is likewise clear that $X \backslash A$ contains no nonempty (1,2)-dense in itself subset and thus $X \backslash A \in(1,2)-\mathcal{S T}(X)$.

The latter theorem shows that the maximal $(1,2)$-dense in itself subset of a $\mathrm{BS}\left(X, \tau_{1}, \tau_{2}\right)$ is both 1- and 2-closed.

Theorem 2.13. In a $\mathrm{BS}\left(X, \tau_{1}<\tau_{2}\right)$ for every set $A \in(1,2)-\mathcal{S T}(X)$ we have $(1,2)-\operatorname{Fr} A \in(1,2)-\mathcal{N D}(X)$.

Proof. By Theorem 2.7 the set $A \cap \tau_{1} \operatorname{int}(1,2)-\operatorname{Fr} A \in(1,2)-\mathcal{D} \mathcal{I}(X)$ and therefore it is empty since $A \in(1,2)-\mathcal{S T}(X)$. Moreover, since $A \cap \tau_{1} \operatorname{int}(1,2)$ -Fr $A$ is 1 -dense in $\tau_{1} \operatorname{int}(1,2)$ - $\operatorname{Fr} A$, the latter set is also empty, i.e., $(1,2)$ - $\operatorname{Fr} A \in$ $\operatorname{co} \tau_{2} \cap 1-\mathcal{B} d(X) \subset(1,2)-\mathcal{N D}(X)$.

By virtue of Theorems 2.5 and 2.10 , in a $\mathrm{BS}\left(X, \tau_{1}<\tau_{2}\right)$ all results that hold for the families $1-\mathcal{D I}(X)$ and $1-\mathcal{S} \mathcal{T}(X)$ also hold for the families $(1,2)-\mathcal{D I}(X)$ and $(1,2)-\mathcal{S} \mathcal{T}(X)$, respectively.

Now we will show when the inverse of the last inclusion in Theorem 2.8 is fair.

The coupling of topologies, i.e., the $C$-relation was defined by J. Weston in [26] to generalize some well-known theorems on topological groups and linear spaces and to connect the same properties of the coupled topologies.

Definition 2.10. A topology $\tau_{1}$ is coupled to a topology $\tau_{2}$ on a set $X$ (briefly, $\tau_{1} C \tau_{2}$ ) if $\tau_{1} \mathrm{cl} U \subset \tau_{2} \operatorname{cl} U$ for every set $U \in \tau_{1}$.

From this definition we immediately find that if $\tau_{1}=\operatorname{co} \tau_{1}$, then $\tau_{1}$ is coupled to every topology on $X$ so that the antidiscrete topology on $X$ as well as the discrete topology on $X$ is coupled to every topology on $X$. In our notations $\tau_{1} C \tau_{2} \wedge \tau_{1} \subset \tau_{2} \Longleftrightarrow \tau_{1}<_{C} \tau_{2}$ and a set $X$ together with the topologies $\tau_{1} C \tau_{2}\left(\tau_{1}<_{C} \tau_{2}\right)$ is denoted by $\left(X, \tau_{1} C \tau_{2}\right)\left(\left(X, \tau_{1}<_{C} \tau_{2}\right)\right)$.

Theorem 2.14. The following conditions are equivalent in a $\operatorname{BS}\left(X, \tau_{1}, \tau_{2}\right)$ :

(1) $\tau_{1}$ is coupled to $\tau_{2}$.

(2) $\tau_{1} \operatorname{cl} \tau_{1} \operatorname{int} A \subset \tau_{2} \operatorname{cl} \tau_{1} \operatorname{int} A$ so that $\tau_{2} \operatorname{int} \tau_{1} \operatorname{cl} A \subset \tau_{1} \operatorname{int} \tau_{1} \operatorname{cl} A$ for every subset $A \subset X$. 
(3) $\tau_{1} \operatorname{cl} \tau_{1} \operatorname{int} A \subset \tau_{2} \operatorname{cl} A$ so that $\tau_{2} \operatorname{int} A \subset \tau_{1} \operatorname{int} \tau_{1} \operatorname{cl} A$ for every subset $A \subset X$.

(4) $\tau_{1}<_{C} \sup \left(\tau_{1}, \tau_{2}\right)$.

(5) For every point $x \in X$ the 1-closure of any 2-neighborhood of $x$ is a 1-neighborhood of $x$.

Proof. The equivalences $(1) \Longleftrightarrow(4) \Longleftrightarrow(5)$ are proved in Theorems 2 and 5 of [26]. (1) $\Longleftrightarrow(2)$ is obvious by Definition 2.10. If (3) is satisfied, then $\tau_{1} \operatorname{cl} A \subset \tau_{2} \mathrm{cl} A$ for every 1-open set $A$ so that $(3) \Longrightarrow(1) \Longleftrightarrow(2)$. Since $(2) \Longrightarrow(3)$ is evident, we obtain $(2) \Longleftrightarrow(3)$.

Corollary. The following conditions are equivalent in a $\mathrm{BS}\left(X, \tau_{1}<\right.$ $\left.\tau_{2}\right)$ :

(1) $\tau_{1}<_{C} \tau_{2}$

(2) $\tau_{1} \operatorname{cl} \tau_{1} \operatorname{int} A=\tau_{2} \operatorname{cl} \tau_{1} \operatorname{int} A$ so that $\tau_{1} \operatorname{int} \tau_{1} \operatorname{cl} A=\tau_{2} \operatorname{int} \tau_{1} \operatorname{cl} A$ for every subset $A \subset X$.

Proof. (1) and (2) are immediate consequences of Definition 2.10 and (2) of Theorem 2.14.

Example 2.6. Let $X=\{a, b, c, d\}, \tau_{1}=\{\varnothing,\{a, b\},\{a, b, c\}, X\}$ and $\tau_{2}=\{\varnothing,\{a, b\},\{a, b, c\},\{a, b, d\}, X\}$. Then $\tau_{1}<_{C} \tau_{2}$. Let us consider the set $F=\{c, d\} \in \operatorname{co} \tau_{1} \subset \operatorname{co} \tau_{2}$. Then for the subset $\{d\}$ of a $\mathrm{BS}\left(Y, \tau_{1}^{\prime}, \tau_{2}^{\prime}\right)$ we have $\tau_{1}^{\prime} \operatorname{int} \tau_{1}^{\prime} \operatorname{cl}\{d\}=\varnothing$ and $\tau_{2}^{\prime} \operatorname{int}\{d\}=\{d\}$, i.e., $\tau_{1}^{\prime}$ is not coupled to $\tau_{2}^{\prime}$.

Definition 2.11. A topology $\tau_{1}$ is $i$-strongly coupled to a topology $\tau_{2}$ on a set $X$ (briefly, $\tau_{1} C(i) \tau_{2}$ ) if the $C$-relation is hereditary with respect to $i$-closed subsets of $X[9]$.

Example 2.7. Let $X=\{a, b, c, d\}, \tau_{1}=\{\varnothing,\{a, b, c\}, X\}$ and $\tau_{2}=$ $\{\varnothing,\{a, b\},\{a, b, c\}, X\}$. Then $\tau_{1}<_{C(i)} \tau_{2}$. $2 \mathcal{P}(X)$.

Theorem 2.15. If $\left(X, \tau_{1}<_{C(2)} \tau_{2}\right)$ is a $1-\mathrm{T}_{1} \mathrm{BS}$, then $\operatorname{co} \tau_{2} \cap 1-\mathcal{D I}(X)=$

Proof. Let $F \in \operatorname{co} \tau_{2} \cap 1-\mathcal{D I}(X)$. Following the condition, $\tau_{1}^{\prime}<_{C} \tau_{2}^{\prime}$ in $\left(F, \tau_{1}^{\prime}, \tau_{2}^{\prime}\right)$ and, by (5) of Corollary 2 of Theorem 2.2.1 in [11], $F_{1}^{i}=F_{2}^{i}=\varnothing$ since $F \in 1-\mathcal{D I}(X)$. Hence $F \subset F_{2}^{d}$, i.e., $F \in 2-\mathcal{D I}(X)$ so that $F \in \operatorname{co} \tau_{2} \cap$ $2-\mathcal{D I}(X)=2-\mathcal{P}(X)$ and thus co $\tau_{2} \cap 1-\mathcal{D I}(X) \subset 2-\mathcal{P}(X)$. The inverse inclusion follows from (2) of Theorem 2.5.

Theorem 2.16. Let $\left(X, \tau_{1}, \tau_{2}\right)$ be $a$ BS and $X \in(1,2)-\mathcal{D} \mathcal{I}(X)$. Then $A \in\left(\tau_{1} \cap \tau_{2}\right) \cup(1 \mathcal{D}(X) \cap 2 \mathcal{D}(X))$ implies that $A \in(1,2)-\mathcal{D} \mathcal{I}(X)$. 
Proof. Assume that $A \in \tau_{1} \cap \tau_{2}$ so that $F=X \backslash A \in \operatorname{co} \tau_{1} \cap \operatorname{co} \tau_{2}$. Then $F_{i}^{d} \subset F$ and thus $(X \backslash F) \backslash(X \backslash F)_{1}^{d} \subset(X \backslash F) \backslash\left(X_{1}^{d} \backslash F_{1}^{d}\right) \subset(X \backslash F) \backslash\left(X_{1}^{d} \backslash F\right)=$ $\left((X \backslash F) \backslash X_{1}^{d}\right) \cup F=((X \backslash F) \cup F) \backslash X_{1}^{d}=X \backslash X_{1}^{d}$. On the other hand, $((X \backslash F) \backslash$ $\left.(X \backslash F)_{1}^{d}\right) \cap F=\varnothing$ and thus $(X \backslash F) \backslash(X \backslash F)_{1}^{d} \subset\left(X \backslash X_{1}^{d}\right) \backslash F$. But the inclusions $X \backslash X_{1}^{d} \subset X_{2}^{d}$ and $F_{2}^{d} \subset F$ imply that $(X \backslash F) \backslash(X \backslash F)_{1}^{d} \subset X_{2}^{d} \backslash F_{2}^{d} \subset(X \backslash F)_{2}^{d}$ so that $A \backslash A_{1}^{d} \subset A_{2}^{d}$, i.e., $A \in(1,2)-\mathcal{D} \mathcal{I}(X)$.

Now let $A \in 1-\mathcal{D}(X) \cap 2-\mathcal{D}(X)$. Then $A_{i}^{d}=X_{i}^{d}$ and $X \in(1,2)-\mathcal{D I}(X) \Longleftrightarrow$ $X \backslash X_{1}^{d} \subset X_{2}^{d}$ implies that $X \backslash A_{2}^{d} \subset A_{1}^{d}$. Therefore $A \backslash A_{2}^{d} \subset A_{1}^{d}$, i.e., $A \in$ $(1,2)-\mathcal{D} \mathcal{I}(X)$.

Corollary. Let $\left(X, \tau_{1}<\tau_{2}\right)$ be $a$ BS and $X \in(1,2)-\mathcal{D I}(X)$. Then $A \in \tau_{1} \cup 2 \mathcal{D}(X)$ implies $A \in(1,2)-\mathcal{D I}(X)$.

We finish the discussion of $(i, j)$-perfect sets with the proof of the bitopological modification of Cantor-Bendixson's theorem. In this context recall that in a BS $\left(X, \tau_{1}<\tau_{2}\right)$ a set $A \in(1,2)-\mathcal{D I}(X)$ if and only if $U \in \tau_{1}$ and $U \cap A \neq \varnothing$ implies $U \cap A$ is infinite, and a point $x \in X$ is an $i$-condensation point of a set $A \in 2^{X}$ if each $i$-open neighborhood $U(x)$ meets $A$ in an uncountable set. The set of all $i$-condensation points of $A$ is denoted by $A_{i}^{0}$.

Theorem 2.17. Let $\left(X, \tau_{1}<\tau_{2}\right)$ be a $1-\mathrm{T}_{1}$ and 1 -second countable BS. Then any uncountable 2 -closed set $F$ contains a set $A \in \operatorname{co} \tau_{2} \cap(1,2)-\mathcal{D I}(X)=$ $(2,1) \mathcal{P}(X)$. Moreover, $F_{1}^{0}=F_{2}^{0}=\tau_{1} \operatorname{cl} A$.

Proof. Let $F \in \operatorname{co} \tau_{2}$ be an uncountable set and $U_{1}, U_{2}, \ldots$ be a countable base of 1-open sets. Suppose that $V_{1}, V_{2}, \ldots$ are those of the sets $U_{1}, U_{2}, \ldots$ which meet $F$ in a countable set. Clearly, the sequence $V_{1}, V_{2}, \ldots$ may be finite or infinite. If $A=F \backslash \bigcup_{k} V_{k}$, then $\tau_{1} \subset \tau_{2}$ implies $A \in \operatorname{co} \tau_{2}$ and $F=\left(A \backslash \underset{k}{\cup} V_{k}\right) \cup\left(\underset{k}{\cup} V_{k} \cap F\right)=A \cup\left(\underset{k}{\cup} V_{k} \cap F\right)$. Since $F$ is uncountable and $\cup_{k} V_{k} \cap F$ is countable, $A=F \backslash \cup_{k} V_{k}$ is uncountable and therefore non-empty.

It remains to prove only that $A \in(1,2)-\mathcal{D} \mathcal{I}(X)$. But But we would rather prove that $A \subset A_{1}^{0}$, i.e., each point $x \in A$ is a 1-condensation point of $A$ so that $U(x) \in \tau_{1}$ gives $|U(x) \cap A|>\aleph_{0}$. Indeed, let $U(x) \in \tau_{1}$ be any neighborhood. Then there is a set $U_{n}$ from the 1-countable base $U_{1}, U_{2}, \ldots$ such that $x \in U_{n} \subset U(x)$. It is evident that $U_{n} \neq V_{k}$ for each $k$ since the contrary implies $x \bar{\epsilon} F \backslash \bigcup_{k} V_{k}=A$. This means that $\left|F \cap U_{n}\right|>\aleph_{0}$. Since $A$ differs from $F$ in merely a countable set, it follows that $\left|A \cap U_{n}\right|>\aleph_{0}$. But $A \cap U_{n} \subset A \cap U(x)$ so that $|A \cap U(x)|>\aleph_{0}$, and hence $A \subset A_{1}^{0}$. Therefore $A \subset A_{1}^{d}$ and thus $A \in \operatorname{co} \tau_{2} \cap(1,2)-\mathcal{D} \mathcal{I}(X)=(2,1)-\mathcal{P}(X)$. Now we have $A \subset$ $A_{1}^{0} \subset A_{1}^{d}$ so that $\tau_{1} \mathrm{cl} A \subset \tau_{1} \mathrm{cl} A_{1}^{0}=A_{1}^{0} \subset \tau_{1} \mathrm{cl} A_{1}^{d}=A_{1}^{d} \subset \tau_{1} \mathrm{cl} A$. Therefore $A_{1}^{0}=\tau_{1} \mathrm{cl} A$. Finally, $F_{1}^{0}=\left(A \cup\left(\underset{k}{\cup} V_{k} \cap F\right)_{1}^{0}=A_{1}^{0} \cup\left(\underset{k}{\cup} V_{k} \cap F\right)_{1}^{0}=A_{1}^{0}=\tau_{1} \mathrm{cl} A\right.$. 
Corollary. Under the hypotheses of Theorem 2.17 for any uncountable set $B \in 2^{X}$ there is an uncountable subset $A \subset B$ such that $A \in(1,2)-\mathcal{D I}(X)$.

Proof. Let $B \in 2^{X}$ be any uncountable set. Then the result follows directly from the proof of Theorem 2.18 omitting the remark that $A$ is 2 closed.

Definition 2.12. A family $\mathcal{B}$ of subsets of a TS $(X, \tau)$ is a pseudobase of the topology $\tau$ if the following conditions are satisfied:

(1) $B \in \mathcal{B} \Longrightarrow \operatorname{int} B \neq \varnothing$.

(2) For every subset $U \in \tau \backslash\{\varnothing\}$ there exists a set $B \in \mathcal{B}$ such that $B \subset U$.

By [25] this definition leads to the equivalence relation on the family of all topologies on a set $X$.

Definition 2.13. Topologies $\tau_{1}$ and $\tau_{2}$ on a set $X$ are $S$-related (briefly, $\left.\tau_{1} S \tau_{2}\right)$ if $\tau_{1} \backslash\{\varnothing\}$ is a pseudobase for $\tau_{2}$.

A set $X$ together with the $S$-related topologies $\tau_{1}$ and $\tau_{2}$ is denoted by $\left(X, \tau_{1} S \tau_{2}\right)$.

Remark 2.2. The $S$-relation between two topologies on a set $X$ is especially important by Proposition 3.4 from [25], following which if one term of the $S$-equivalence class is Baire, then all members of this class are also Baire.

Example 2.8. Let $(\mathbb{R}, s, \tau)$ be a BS, where $s$ is the half-open interval topology, i.e., the Sorgenfrey topology on $\mathbb{R}$ so that basic open sets for $s$ are of the form $[a, b)$ and $\tau$ is the topology with basic open sets of the form $(a, b]$. It is clear that neither topology is finer than the other, $\inf (s, \tau)=s \cap \tau=\omega$ is the natural topology on $\mathbb{R}, \sup (s, \tau)$ is the discrete topology on $\mathbb{R}$. Moreover, $s S \tau, s S \inf (s, \tau)$ and $\tau S \inf (s, \tau)$. Hence, by Remark 2.2, the Sorgenfrey line, i.e., $\mathbb{R}$ with the Sorgenfrey topology is a Baire space since $s S \inf (s, \tau)$, and the natural topology is Baire [25].

Theorem 2.18. The following conditions are equivalent in a $B S$ $\left(X, \tau_{1}, \tau_{2}\right)$ :

(1) $\tau_{1}$ is $S$-related to $\tau_{2}$.

(2) $1-\mathcal{B} d(X)=2-\mathcal{B} d(X)=(1,2)-\mathcal{B} d(X)=(2,1)-\mathcal{B} d(X)$ so that

$1 \mathcal{D}(X)=2 \mathcal{D}(X)=(1,2) \mathcal{D}(X)=(2,1) \mathcal{D}(X)$.

(3) $\tau_{1} \operatorname{int} A \subset \tau_{1} \operatorname{cl} \tau_{2} \operatorname{int} A \wedge \tau_{2} \operatorname{int} A \subset \tau_{2} \operatorname{cl} \tau_{1} \operatorname{int} A$ so that

$\tau_{1}$ int $\tau_{2} \operatorname{cl} A \subset \tau_{1} \operatorname{cl} A \wedge \tau_{2}$ int $\tau_{1} \operatorname{cl} A \subset \tau_{2} \operatorname{cl} A$ for every subset $A \subset X$.

(4) $\tau_{1} \subset(2,1)-\mathcal{S O}(X) \wedge \tau_{2} \subset(1,2)-\mathcal{S O}(X)$ so that $\operatorname{co} \tau_{1} \subset(2,1)-\mathcal{S C}(X) \wedge \operatorname{co} \tau_{2} \subset(1,2)-\mathcal{S C}(X)$. 
Proof. It is clear that $(1-\mathcal{B} d(X)=2-\mathcal{B} d(X)) \Longleftrightarrow\left(\tau_{1}\right.$ int $A \neq \varnothing \Longleftrightarrow$ $\tau_{2}$ int $A \neq \varnothing$ for every subset $\left.A \subset X\right)$. Thus, by Proposition 1.1.2 in [11], for the equivalence $(1) \Longleftrightarrow(2)$ it is enough to show that $(1) \Longleftrightarrow\left(\tau_{1}\right.$ int $A \neq$ $\varnothing \Longleftrightarrow \tau_{2}$ int $A \neq \varnothing$ for every subset $\left.A \subset X\right)$.

We assume that $\tau_{1} S \tau_{2}$, i.e., $\tau_{1} \backslash\{\varnothing\}$ is a pseudobase for $\tau_{2}$, and $A \subset X$ is any subset. If $\tau_{1} \operatorname{int} A \neq \varnothing$, then, by (1) of Definition 2.12, $\tau_{2}$ int $\tau_{1} \operatorname{int} A \neq \varnothing$ and hence $\tau_{2}$ int $A \neq \varnothing$. When $\tau_{2}$ int $A \neq \varnothing$, by (2) of Definition 2.12 there exists a set $V \in \tau_{1} \backslash\{\varnothing\}$ such that $V \subset \tau_{2} \operatorname{int} A$. Thus $\tau_{1} \operatorname{int} \tau_{2} \operatorname{int} A \neq \varnothing$ so that $\tau_{1}$ int $A \neq \varnothing$.

On the other hand, let $U \in \tau_{1} \backslash\{\varnothing\}$. Then $\tau_{2}$ int $U \neq \varnothing$. If $U \in \tau_{2} \backslash\{\varnothing\}$, we have $\varnothing \neq V=\tau_{1} \operatorname{int} U \subset U$ and consequently $\tau_{1} \backslash\{\varnothing\}$ is a pseudobase for $\tau_{2}$.

Therefore $(1) \Longleftrightarrow(2)$.

The implication $(1) \Longrightarrow(3)$ is exactly Proposition 3.3 from [25] and $(3) \Longrightarrow(2) \Longleftrightarrow(1)$ is obvious.

$(3) \Longleftrightarrow(4)$ is an immediate consequence of Theorem 1.3.3 in [11].

It is likewise easy to see that if $\tau_{1}=\omega$ is the natural topology on $\mathbb{R}$ and $\tau_{2}$ is the discrete topology on $\mathbb{R}$, then $\tau_{1}$ is not $S$-related to $\tau_{2}$.

Note that in the sequel it will be assumed that $\tau_{1} S \tau_{2} \wedge \tau_{1} \subset \tau_{2} \Longleftrightarrow \tau_{1}<_{S} \tau_{2}$ and the coresponding BS will be denoted by $\left(X, \tau_{1}<_{S} \tau_{2}\right)$.

Remark 2.3. It is not difficult to see that $\tau_{1}<_{S} \tau_{2} \Longleftrightarrow \tau_{1} \subset \tau_{2} \subset$ $(1,2)-\mathcal{S O}(X) \subset(2,1)-\mathcal{S O}(X)$ since $\tau_{1} \subset \tau_{2} \Longrightarrow(1,2)-\mathcal{S O}(X) \subset(2,1)-\mathcal{S O}(X)$.

Definition 2.14. A topology $\tau_{1}$ is near a topology $\tau_{2}$ on a set $X$ (briefly, $\left.\tau_{1} N \tau_{2}\right)$ if $\tau_{1} \mathrm{cl} U \subset \tau_{2} \mathrm{cl} U$ for every set $U \in \tau_{2}$ [9].

Example 2.9. Let $\left(\mathbb{R}, \omega_{1}, \omega_{2}\right)$ be the natural BS [11]. Then $\omega_{i}$ is neither $S$-related to $\omega_{j}$ nor coupled to $\omega_{j}$; however $\omega_{i}$ is near $\omega_{j}$.

In contrast to the $S$-relation, the $N$-relation, like the $C$-relation, is not symmetric.

Example 2.10. Let $X=\{a, b, c\}, \tau_{1}=\{\varnothing,\{a, b\}, X\}$ and $\tau_{2}=\{\varnothing,\{a\}$, $\{b\},\{a, b\},\{b, c\}, X\}$. Then $\tau_{2} N \tau_{1}$ since $\tau_{1} \subset \tau_{2}$, but $\tau_{1}$ is not near $\tau_{2}$ since for the set $\{b, c\} \in \tau_{2}$ we have $\tau_{1} \operatorname{cl}\{b, c\}=X$ and $\tau_{2} \operatorname{cl}\{b, c\}=\{b, c\}$.

It is evident that $\tau_{1}<_{N} \tau_{2} \Longleftrightarrow \tau_{1} N \tau_{2} \wedge \tau_{1} \subset \tau_{2}$.

Example 2.11. Let $X$ be any set such that $a, b \in X, a \neq b$, implies that $X \backslash\{a, b\} \neq \varnothing$. If $\tau_{1}=\{\varnothing,\{a\}, X \backslash\{a\}, X\}, \tau_{2}=\{\varnothing,\{a\}, X \backslash\{a\},\{b\}$, $\{a, b\}, X\}$, then $\tau_{1}<_{N} \tau_{2}$.

Theorem 2.19. The following conditions are equivalent in a $\mathrm{BS}$ $\left(X, \tau_{1}, \tau_{2}\right)$ : 
(1) $\tau_{1}$ is near $\tau_{2}$.

(2) $\tau_{1} \operatorname{cl} \tau_{2} \operatorname{int} A \subset \tau_{2} \operatorname{cl} \tau_{2} \operatorname{int} A$ so that $\tau_{2}$ int $\tau_{2} \operatorname{cl} A \subset \tau_{1} \operatorname{int} \tau_{2} \operatorname{cl} A$ for every subset $A \subset X$.

(3) $\tau_{1} \operatorname{cl} \tau_{2} \operatorname{int} A \subset \tau_{2} \operatorname{cl} A$ so that $\tau_{2} \operatorname{int} A \subset \tau_{1} \operatorname{int} \tau_{2} \operatorname{cl} A$ for every subset $A \subset X$.

(4) For every point $x \in X$ the 2-closure of any 2-neighborhood of $x$ is a 1-neighborhood of $x$.

Proof. It is obvious that (1) $\Longleftrightarrow(2) \Longrightarrow(3)$. If (3) is satisfied, then $\tau_{1} \operatorname{cl} U \subset \tau_{2} \mathrm{cl} U$ for every set $U \in \tau_{2}$, i.e., $(3) \Longrightarrow(1)$.

$(1) \Longrightarrow(4)$ Let $x \in X$ be any point and $U(x)$ be its any 2-neighborhood. It can be assumed without loss of generality that $U(x) \in \tau_{2}$. Then $V=$ $X \backslash \tau_{2} \operatorname{cl} U(x) \in \tau_{2}$ and, by (1), $\tau_{1} \operatorname{cl} V \subset \tau_{2} \operatorname{cl} V$. If $V_{1}=X \backslash \tau_{1} \operatorname{cl} V$, then $V_{1}=X \backslash \tau_{1} \operatorname{cl}\left(X \backslash \tau_{2} \operatorname{cl} U(x)\right)=\tau_{1}$ int $\tau_{2} \operatorname{cl} U(x) \subset \tau_{2} \operatorname{cl} U(x)$. Furthermore, $x \in$ $\tau_{2} \operatorname{cl} U(x) \Longrightarrow x \bar{\epsilon} X \backslash \tau_{2} \operatorname{cl} U(x)=\tau_{2} \operatorname{int}(X \backslash U(x))$. Since $\tau_{2} \operatorname{int}(X \backslash U(x)) \cap$ $U(x)=\varnothing$, we obtain $\tau_{2} \operatorname{cl} \tau_{2} \operatorname{int}(X \backslash U(x)) \cap U(x)=\varnothing$. Hence $x \bar{\epsilon}$ $\tau_{2} \operatorname{cl}\left(X \backslash \tau_{2} \operatorname{cl} U(x)\right)=\tau_{2} \operatorname{cl} V$ and $\tau_{1} \operatorname{cl} V \subset \tau_{2} \operatorname{cl} V$ implies $x \bar{\epsilon} \tau_{1} \operatorname{cl} V$ so that $x \in V_{1}=X \backslash \tau_{1} \mathrm{cl} V$. Thus $V_{1}$ is a 1-open neighborhood of $x$ such that $V_{1} \subset \tau_{2} \operatorname{cl} U(x)$.

(4) $\Longrightarrow(1)$ Suppose that $U \in \tau_{2}$ is any set and $x \in \tau_{1} \operatorname{cl} U$ is an arbitrary point. If $U(x)$ is any 2-open neighborhood of $x$, then by (4) there exists a set $V \in \tau_{1}$ such that $x \in V \subset \tau_{2} \operatorname{cl} U(x)$. It is clear that $V \cap U \neq \varnothing$ so that $\tau_{2} \operatorname{cl} U(x) \cap U \neq \varnothing$. Hence $U(x) \cap U \neq \varnothing$ and $U(x) \in \tau_{2}$ being arbitrary implies that $x \in \tau_{2} \operatorname{cl} U$.

Corollary. For a BS $\left(X, \tau_{1}, \tau_{2}\right)$ we have

$\tau_{1} \operatorname{cl} U=\tau_{2} \operatorname{cl} U$ for every set $U \in \tau_{2} \Longleftrightarrow \tau_{1}<_{N} \tau_{2} \Longrightarrow \tau_{1} \operatorname{cl} V=\tau_{2} \operatorname{cl} V$ for every set $V \in \tau_{1} \Longleftrightarrow \tau_{1}<_{C} \tau_{2}$.

A nontrivial example of the near topologies is given in [3]: if $\left(X, \tau_{1}\right)$ is a compact space with the first axiom of countability, where $|U|>\aleph_{0}$ for each $U \in \tau_{1} \backslash\{\varnothing\}$ and $\tau_{2}=\left\{U \backslash A: U \in \tau_{1}, A \subset X,|A| \leq \aleph_{0}\right\}$, then $\tau_{1} \subset \tau_{2}$ and the $\operatorname{BS}\left(X, \tau_{1}<\tau_{2}\right)$ is $\left(X, \tau_{1}<_{N} \tau_{2}\right)$.

At the end of this paragraph we will consider three operators on $2^{X}$, characterizing not only the degrees of nearness of the four boundaries of a set and the $S$-, $C$ - and $N$-relations, but also interrelations of the dimension functions in $\S 2$.

Definition 2.15. In a BS $\left(X, \tau_{1}<\tau_{2}\right)$ the indicators of near"ess of the boundaries are the"following three operators: $\mathbf{n}_{1}, \mathbf{n}_{2}, \mathbf{n}: 2^{X} \rightarrow(2,1)-\mathcal{L C}(X)$ defined as follows: $\mathbf{n}_{1}(A)=\tau_{1} \operatorname{cl} A \backslash \tau_{2} \operatorname{cl} A, \mathbf{n}_{2}(A)=\tau_{2} \operatorname{int} A \backslash \tau_{1} \operatorname{int} A$ and $\mathbf{n}(A)=\mathbf{n}_{1}(A) \cup \mathbf{n}_{2}(A)$ for each set $A \in 2^{X}$. 
It is evident that $\mathbf{n}_{i}(A)=\mathbf{n}_{j}(X \backslash A)$ so that $\mathbf{n}(A)=\mathbf{n}(X \backslash A)$ for each set $A \in 2^{X}, \mathbf{n}_{1} /$ co $\tau_{1}=\mathbf{n}_{2} / \tau_{1}=\mathbf{n} / \tau_{1} \cap$ co $\tau_{1}=\varnothing$ and therefore $\mathbf{n} / \tau_{1}=\mathbf{n}_{1}$, $\mathbf{n} /$ со $\tau_{1}=\mathbf{n}_{2}$.

Theorem 2.20. In a $\mathrm{BS}\left(X, \tau_{1}<\tau_{2}\right)$ the operators $\mathbf{n}_{1}, \mathbf{n}_{2}$ and $\mathbf{n}$ satisfy the following conditions:

(1) $(1,2)$ - $F r A=2$-Fr $A \cup \mathbf{n}_{1}(A)=2$-Fr $A \triangle \mathbf{n}_{1}(A),(2,1)$-Fr $A=2$-Fr $A \cup$ $\mathbf{n}_{2}(A)=2$-Fr $A \triangle \mathbf{n}_{2}(A), 1-\mathrm{Fr} A=(1,2)-\mathrm{Fr} A \cup \mathbf{n}_{2}(A)=(1,2)$-Fr $A \triangle \mathbf{n}_{2}(A)=(2,1)-\operatorname{Fr} A \cup \mathbf{n}_{1}(A)=(2,1)-\operatorname{Fr} A \triangle \mathbf{n}_{1}(A)=2$-Fr $A \cup \mathbf{n}(A)=$ 2 -Fr $A \triangle \mathbf{n}(A)$ for each set $A \in 2^{X}$.

(2) $A \in \tau_{1} \cap\left(\operatorname{co} \tau_{2} \backslash \operatorname{co} \tau_{1}\right) \Longrightarrow \mathbf{n}(A)=\mathbf{n}_{1}(A)=1$-Fr $A=(1,2)-\operatorname{Fr} A$, $(2,1)-\operatorname{Fr} A=2-\operatorname{Fr} A=\varnothing$.

$A \in\left(\tau_{2} \backslash \tau_{1}\right) \cap \operatorname{co} \tau_{1} \Longrightarrow \mathbf{n}(A)=\mathbf{n}_{2}(A)=1-\operatorname{Fr} A=(2,1)-\operatorname{Fr} A,(1,2)-\operatorname{Fr} A=$ 2 -Fr $A=\varnothing$.

$A \in\left(\tau_{2} \backslash \tau_{1}\right) \cap\left(\operatorname{co} \tau_{2} \backslash \operatorname{co} \tau_{1}\right) \Longrightarrow \mathbf{n}_{1}(A)=(1,2)-\operatorname{Fr} A, \mathbf{n}_{2}(A)=(2,1)-\operatorname{Fr} A$, $\mathbf{n}(A)=1$-Fr $A, 2$-Fr $A=\varnothing$.

(3) $A \in 2-\mathcal{O D}(X) \Longrightarrow \mathbf{n}_{1}(A) \in(1,2)-\mathcal{N D}(X), A \in(1,2)-\mathcal{O D}(X) \Longrightarrow$ $\mathbf{n}_{1}(A) \in 1-\mathcal{N D}(X), A \in(2,1)-\mathcal{O D}(X) \Longrightarrow \mathbf{n}_{1}(A) \in 2-\mathcal{N D}(X), A \in$ $(1,2)-\mathcal{O D}(X) \cap(1,2)-\mathcal{S C}(X) \Longrightarrow \mathbf{n}_{1}(A) \in(2,1)-\mathcal{N D}(X)$ so that $A \in 2-\mathcal{C D}(X) \Longrightarrow \mathbf{n}_{2}(A) \in(1,2)-\mathcal{N D}(X), A \in(1,2)-\mathcal{C D}(X) \Longrightarrow$ $\mathbf{n}_{2}(A) \in 1-\mathcal{N} \mathcal{D}(X), A \in(2,1)-\mathcal{C D}(X) \Longrightarrow \mathbf{n}_{2}(A) \in 2-\mathcal{N D}(X), A \in$ $(1,2)-\mathcal{C D}(X) \cap(1,2)-\mathcal{S O}(X) \Longrightarrow \mathbf{n}_{2}(A) \in(2,1)-\mathcal{N D}(X)$.

(4) $\tau_{1} C \tau_{2} \Longleftrightarrow \mathbf{n}_{1}(U)=\varnothing$ for each set $U \in \tau_{1} \Longleftrightarrow \mathbf{n}_{2}(F)=\varnothing$ for each set $F \in \operatorname{co} \tau_{1}$.

$\tau_{1} N \tau_{2} \Longleftrightarrow \mathbf{n}_{1}(U)=\varnothing$ for each set $U \in \tau_{2} \Longleftrightarrow \mathbf{n}_{2}(F)=\varnothing$ for each set $F \in \operatorname{co} \tau_{2}$.

$\tau_{1} S \tau_{2} \Longleftrightarrow \mathbf{n}_{1}(A) \in 2-\mathcal{B} d(X)$ for each set $A \in 2^{X} \Longleftrightarrow \mathbf{n}_{2}(A) \in 2-\mathcal{B} d(X)$ for each set $A \in 2^{X} \Longleftrightarrow \mathbf{n}(A) \in 2-\mathcal{B d}(X)$ for each set $A \in 2^{X}$.

(5) $A \in(2,1)-\mathcal{D I}(X)=(1,2)-\mathcal{D I}(X)=1-\mathcal{D I}(X) \Longleftrightarrow A_{2}^{i} \subset \mathbf{n}_{1}(A)$.

Proof. (1) (1,2)-Fr $A=\tau_{1} \operatorname{cl} A \cap \tau_{2} \operatorname{cl}(X \backslash A)=\left(\mathbf{n}_{1}(A) \cup \tau_{2} \operatorname{cl} A\right) \cap$ $\tau_{2} \operatorname{cl}(X \backslash A)=2$-Fr $A \cup \mathbf{n}_{1}(A)=2$-Fr $A \triangle \mathbf{n}_{1}(A)$. By (4) of Theorem 1.3.1 in [11], $(2,1)$ - $\operatorname{Fr} A=(1,2)-\operatorname{Fr}(X \backslash A)=2-\operatorname{Fr}(X \backslash A) \cup \mathbf{n}_{1}(X \backslash A)=2$-Fr $A \cup$ $\mathbf{n}_{2}(A)=2$-Fr $A \triangle \mathbf{n}_{2}(A)$. 1-Fr $A=\tau_{1} \operatorname{cl} A \cap \tau_{1} \operatorname{cl}(X \backslash A)=\tau_{1} \operatorname{cl} A \cap\left(\mathbf{n}_{1}(X \backslash A) \cup\right.$ $\left.\tau_{2} \operatorname{cl}(X \backslash A)\right)=(1,2)-\operatorname{Fr} A \cup \mathbf{n}_{2}(A)=(1,2)-\operatorname{Fr} A \triangle \mathbf{n}_{2}(A)=\left(\mathbf{n}_{1}(A) \cup \tau_{2} \operatorname{cl} A\right) \cap$ $\tau_{1} \operatorname{cl}(X \backslash A)=(2,1)-\operatorname{Fr} A \cup \mathbf{n}_{1}(A)=(2,1)-F r A \triangle \mathbf{n}_{1}(A)=\left(\mathbf{n}_{1}(A) \cup \tau_{2} \operatorname{cl} A\right) \cap$ $\left(\mathbf{n}_{1}(X \backslash A) \cup \tau_{2} \operatorname{cl}(X \backslash A)\right)=2$-Fr $A \cup \mathbf{n}(A)=2$-Fr $A \triangle \mathbf{n}(A)$.

(2) $A \in \tau_{1} \cap\left(\operatorname{co} \tau_{2} \backslash \operatorname{co} \tau_{1}\right) \Longrightarrow \mathbf{n}(A)=\mathbf{n}_{1}(A)=\tau_{1} \operatorname{cl} A \cap(X \backslash A)=$ $\tau_{1} \operatorname{cl} A \cap \tau_{1} \operatorname{cl}(X \backslash A)=1-F r A=\tau_{1} \operatorname{cl} A \cap \tau_{2} \operatorname{cl}(X \backslash A)=(1,2)-F r A,(2,1)-F r A=$ 2 - $\operatorname{Fr} A=\varnothing$ is evident. 
$A \in\left(\tau_{2} \backslash \tau_{1}\right) \cap \operatorname{co} \tau_{1} \Longrightarrow \mathbf{n}(A)=\mathbf{n}_{2}(A)=A \cap \tau_{1} \operatorname{cl}(X \backslash A)=\tau_{1} \operatorname{cl} A \cap$ $\tau_{1} \operatorname{cl}(X \backslash A)=1-\operatorname{Fr} A=\tau_{2} \operatorname{cl} A \cap \tau_{1} \operatorname{cl}(X \backslash A)=(2,1)-\operatorname{Fr} A,(1,2)-\operatorname{Fr} A=2$-Fr $A=$ $\varnothing$ is evident.

$A \in\left(\tau_{2} \backslash \tau_{1}\right) \cap\left(\operatorname{co} \tau_{2} \backslash \operatorname{co} \tau_{1}\right) \Longrightarrow \mathbf{n}_{1}(A)=\tau_{1} \operatorname{cl} A \cap(X \backslash A)=\tau_{1} \operatorname{cl} A \cap$ $\tau_{2} \operatorname{cl}(X \backslash A)=(1,2)-\operatorname{Fr} A, \mathbf{n}_{2}(A)=A \cap \tau_{1} \operatorname{cl}(X \backslash A)=\tau_{2} \operatorname{cl} A \cap \tau_{1} \operatorname{cl}(X \backslash A)=$ $(2,1)-\operatorname{Fr} A, \mathbf{n}(A)=(1,2)-\operatorname{Fr} A \cup(2,1)-\operatorname{Fr} A=(1,2)-\operatorname{Fr} A \triangle(2,1)-\operatorname{Fr} A=1$-Fr $A$, 2 -Fr $A=\varnothing$ is evident.

(3) The proof consists of elementary calculations taking into account the equality $\mathbf{n}_{1}(A)=\mathbf{n}_{2}(X \backslash A)$ for each set $A \in 2^{X}$.

(4) The equivalences for $\tau_{1} C \tau_{2}$ and $\tau_{1} N \tau_{2}$ are immediate consequences of the corresponding Definitions 2.10 and 1.14.

If $\tau_{1} S \tau_{2}$, then, by (b) of 4.A.2 in [18], $\mathbf{n}_{1}(A) \in 2-\mathcal{N D}(X)$ and $\mathbf{n}_{2}(A) \in$ $2-\mathcal{N D}(X)$ for each set $A \in 2^{X}$. Therefore $\mathbf{n}_{1}(A) \in 2-\mathcal{B} d(X), \mathbf{n}_{2}(A) \in 2-\mathcal{B} d(X)$ for each set $A \in 2^{X}$ and thus $\mathbf{n}(A) \in 2-\mathcal{B} d(X)$ for each set $A \in 2^{X}$.

Conversely, $\mathbf{n}(A) \in 2-\mathcal{B} d(X)$ for each set $A \in 2^{X} \Longrightarrow \mathbf{n}_{1}(A) \in 2-\mathcal{B} d(X)$ and $\mathbf{n}_{2}(A) \in 2-\mathcal{B} d(X)$ for each set $A \in 2^{X} \Longrightarrow \tau_{2} \operatorname{int}\left(\tau_{2} \operatorname{int} A \backslash \tau_{1} \operatorname{int} A\right)=\varnothing$ for each set $A \in 2^{X} \Longrightarrow \tau_{2} \operatorname{int} A \subset \tau_{2} \operatorname{cl} \tau_{1} \operatorname{int} A$ for each set $A \in 2^{X} \Longrightarrow \tau_{2} \subset$ $(1,2)-\mathcal{S O}(X)$ and by Remark 2.3, $\tau_{1} S \tau_{2}$.

(5) $A \in(2,1)-\mathcal{D I}(X) \Longrightarrow A_{2}^{i} \subset A_{1}^{d}=\left(A_{1}^{d} \backslash A_{2}^{d}\right) \cup A_{2}^{d} \Longrightarrow A_{2}^{i} \subset$ $\left(A_{1}^{d} \backslash A_{2}^{d}\right)=\left(A \cup A_{1}^{d}\right) \backslash\left(A \cup A_{2}^{d}\right)=\mathbf{n}_{1}(A)$. Conversely, $A_{2}^{i} \subset \mathbf{n}_{1}(A) \Longrightarrow A_{2}^{i} \subset$ $A_{1}^{d} \backslash A_{2}^{d} \subset A_{1}^{d}$ so that $A \in(2,1)-\mathcal{D} \mathcal{I}(X)$.

\section{Dimension of Bitopological Spaces}

In this paragraph, generalizing the notions of topological dimension, we will assign a nonnegative integer to certain BS's. Unlike the topological case, the following six numbers will be assigned to a $\mathrm{BS}\left(X, \tau_{1}, \tau_{2}\right):(i, j)$-ind $X$, $p$-ind $X,(i, j)$-Ind $X$ and $p$-Ind $X$.

We give in two distinct ways the equivalent definitions of the pairwise small inductive dimension $p$-ind $X$ for every nonnegative integer $n$.

Definition 3.1. Let $(x, A)$ be a pair in a $\mathrm{BS}\left(X, \tau_{1}, \tau_{2}\right)$ such that $A \in$ $\operatorname{co} \tau_{i}$ and $x \bar{\epsilon} A$. Then a partition corresponding to the pair $(x, A)$ is a p-closed set $T$ for which $X \backslash T$ is not $p$-connected so that $X \backslash T=H$, where $H=H_{1} \cup H_{2}$, $H_{i} \in \tau_{i} \backslash\{\varnothing\}, H_{1} \cap H_{2}=\varnothing$ and $x \in H_{i}, A \subset H_{j}$.

Remark 3.1. It is easy to verify that in a $\mathrm{BS}\left(X, \tau_{1}, \tau_{2}\right)$ the following conditions are satisfied for a pair $(x, A)$, where $A \in \operatorname{co} \tau_{i}, x \bar{\epsilon} A$ :

(1) If there exists an $i$-open neighborhood $U(x)$ (a j-open neighborhood $U(A)$ ) such that $\tau_{j} \operatorname{cl} U(x) \subset X \backslash A\left(\tau_{i} \operatorname{cl} U(A) \subset X \backslash\{x\}\right)$, then the set $(j, i)$ $\operatorname{Fr} U(x)((i, j)-\operatorname{Fr} U(A))$ is the partition corresponding to $(x, A)$ in the sense of Definition 2.1. 
(2) If $T$ is a partition corresponding to $(x, A)$ in the sense of Definition 2.1, then $(j, i)$ - $\operatorname{Fr} H_{i} \subset T$.

Indeed, (1) $X \backslash(j, i)$ - $F r U(x)=X \backslash\left(\tau_{j} \operatorname{cl} U(x) \cap(X \backslash U(x))=\left(X \backslash \tau_{j} \mathrm{cl}\right.\right.$ $U(x)) \cup U(x)$, where $x \in U(x) \in \tau_{i}, A \subset X \backslash \tau_{j} \operatorname{cl} U(x) \in \tau_{j}$ and $U(x) \cap$ $\left(X \backslash \tau_{j} \operatorname{cl} U(x)=\varnothing\right.$. The case in the brackets can be proved in a similar manner.

(2) Since $\left(X \backslash T, \tau_{1}^{\prime}, \tau_{2}^{\prime}\right)$ is not $p$-connected we have $H_{i} \in \tau_{i}^{\prime} \cap \operatorname{co} \tau_{j}^{\prime}$ and hence $(j, i)$-Fr $H_{i} \cap(X \backslash T)=\left(\tau_{j} \operatorname{cl} H_{i} \cap(X \backslash T)\right) \cap\left(\left(X \backslash H_{i}\right) \cap(X \backslash T)\right)=\tau_{j}^{\prime} \operatorname{cl} H_{i} \cap$ $H_{j}=H_{i} \cap H_{j}=\varnothing$ so that $(j, i)-$ Fr $H_{i} \subset T$.

Definition 3.2. Let $\left(X, \tau_{1}, \tau_{2}\right)$ be $a$ BS and $n$ denote a nonnegative integer. We say that

(1) $(i, j)$-ind $X=-1 \Longleftrightarrow X=\varnothing$.

(2) $(i, j)$-ind $X \leq n$ if to every pair $(x, A)$, where $A \in \operatorname{co} \tau_{i}, x \bar{\in} A$, there corresponds a partition $T$ such that $(i, j)$-ind $T \leq n-1$.

(3) $(i, j)$-ind $X=n$ if $(i, j)$-ind $X \leq n$ and the inequality $(i, j)$-ind $X \leq n-1$ does not hold.

(4) $(i, j)$-ind $X=\infty$ if the inequality $(i, j)$-ind $X \leq n$ does not hold for any $n$.

As a rule, $p$-ind $X \leq n \Longleftrightarrow(1,2)$-ind $X \leq n \wedge(2,1)$-ind $X \leq n$.

This definition immediately yields some simple properties of the introduced functions.

Proposition 3.1. The following statements hold in a BS $\left(X, \tau_{1}, \tau_{2}\right)$ :

(1) If $(i, j)$-ind $X$ is finite, then $\left(X, \tau_{1}, \tau_{2}\right)$ is $(i, j)$-regular. Therefore $\left(\tau_{1}<_{C}\right.$

$\tau_{2} \wedge(2,1)$-ind $\left.X<\infty\right)$ or $\left(\tau_{1}<_{N} \tau_{2} \wedge 2\right.$-ind $\left.X<\infty\right) \Longrightarrow \tau_{1}=\tau_{2}$.

(2) If $\left(Y, \tau_{1}^{\prime}, \tau_{2}^{\prime}\right)$ is any $\operatorname{BsS}$ of $\left(X, \tau_{1}, \tau_{2}\right)$, then $(i, j)$-ind $\left.Y \leq(i, j)\right)$-ind $X$.

Proof. (1) Let $(i, j)$-ind $X=n<\infty$. If $x \in X, F \in \operatorname{co} \tau_{i}$ and $x \bar{\epsilon} F$, then by (2) of Definition 2.2 there exists a partition $T$, i.e., $X \backslash T=H_{1} \cup H_{2}$, $H_{i} \in \tau_{i} \backslash\{\varnothing\}, x \in H_{i}, A \subset H_{j}$ and $H_{i} \cap H_{j}=\varnothing$. Therefore $\left(X, \tau_{1}, \tau_{2}\right)$ is $(i, j)$ regular. For the rest of the proof it remains to use Corollary 3 of Theorem 2.2.1 in [11] and Theorem 1 in [26], respectively.

(2) It suffices to prove that $(i, j)$-ind $X=k$ implies $(i, j)$-ind $Y \leq k$. For $k=-1, k=\infty$, the statement is correct. Let it be also correct for $k \leq n-1$. We will prove the statement for $k=n$. If $x \in Y, A^{\prime} \in \operatorname{co} \tau_{i}^{\prime}$ and $x \bar{\in} A^{\prime}$, then there exists a set $A \in \operatorname{co} \tau_{i}$ such that $A^{\prime}=A \cap Y$. Since $(i, j)$-ind $X=n$, to the pair $(x, A)$ there corresponds a partition $T$ such that $(i, j)$-ind $T \leq n-1$. It is obvious that $T^{\prime}=T \cap Y$ is the partition corresponding to $\left(x, A^{\prime}\right)$ in $\left(Y, \tau_{1}^{\prime}, \tau_{2}^{\prime}\right)$. Hence by the inductive assumption $(i \square j)$-ind $T^{\prime} \leq n-1$ as $T^{\prime} \subset T$.

Corollary 3.1. The following statements hold in $\mathrm{BS}\left(X, \tau_{1}, \tau_{2}\right)$ : 
(1) If $p$-ind $X$ is finite, then $\left(X, \tau_{1}, \tau_{2}\right)$ is p-regular.

(2) If $\left(Y, \tau_{1}^{\prime}, \tau_{2}^{\prime}\right)$ is any $\operatorname{BsS}$ of $\left(X, \tau_{1}, \tau_{2}\right)$, then $p$-ind $Y \leq p$-ind $X$.

Corollary 3.2. Let $\left(X, \tau_{1}, \tau_{2}\right)$ be a $\mathrm{BS}$ and $n$ denote a nonnegative integer. Then

(1) $(i, j)$-ind $X \leq n \Longleftrightarrow$ for every point $x \in X$ and any $i$-neighborhood $U(x)$ there exists an $i$-open neighborhood $V(x)$ such that $\tau_{j} \operatorname{cl} V(x) \subset U(x)$ and $(i, j)-\operatorname{ind}(j, i)-\operatorname{Fr} V(x) \leq n-1 \Longleftrightarrow\left(X, \tau_{1}, \tau_{2}\right)$ has an $i$-base $\left.\mathcal{B}\right\rangle$ such that $(i, j)$-ind $(j, i)-\mathrm{Fr} V \leq n-1$ for every $V \in \mathcal{B}_{\rangle}$.

(2) If $\left(X, \tau_{1}, \tau_{2}\right)$ is $i$-second countable, then $(i, j)$-ind $X \leq n \Longleftrightarrow\left(X, \tau_{1}, \tau_{2}\right)$ has a countable $i$-base $\mathcal{B}_{i}$ such that $(i, j)$-ind $(j, i)-\operatorname{Fr} V \leq n-1$ for every $V \in \mathcal{B}_{i}$.

Proof. It sufficessto prove only the first equivalence in (1). Let $(i, j)$ ind $X \leq n, x \in X$ and $U(x)$ be any $i$-neighborhood. It can be assumed without loss of generality that $U(x) \in \tau_{i}$. Then for the pair $(x, A=X \backslash U(x))$ there exists a partition $T$ such that $(i, j)$-ind $T \leq n-1, X \backslash T=H_{1} \cup H_{2}$, $H_{i} \in \tau_{i} \backslash\{\varnothing\}, x \in H_{i}, A \subset H_{j}$ and $H_{1} \cap H_{2}=\varnothing$. It is evident that $\tau_{j} \mathrm{cl} H_{i} \cap$ $H_{j}=\varnothing$ so that $\tau_{j} \operatorname{cl} H_{i} \subset X \backslash A=U(x)$. Let $H_{i}=V(x)$. Then $x \in V(x) \subset$ $\tau_{j} \operatorname{cl} V(x) \subset U(x)$ and, by (2) of Remark 2.1 and (2) of Proposition 2.1, $(i, j)$-ind $(j, i)-\operatorname{Fr} V(x) \leq n-1$.

Conversely, let the condition in the right-hand part of the equivalence be satisfied and $(x, A)$, where $A \in \operatorname{co} \tau_{i}, x \bar{\in} A$ behany pair. Then $U(x)=X \backslash A$ is an $i$-open neighborhood of $x$ and, by condition, there exists an $i$-open neighborhood $V(x)$ such that $\tau_{j} \operatorname{cl} V(x) \subset U(x)$ and $(i, j)-\operatorname{ind}(j, i)-\operatorname{Fr} V(x) \leq n-1$. But following (1) of Remark 2.1, $(j, i)$ - Fr $V(x)$ is the partition corresponding to $(x, A)$ and thus it remains to apply (2) of Definition 2.2.

Proposition 3.2. If for a $\mathrm{BS}\left(X, \tau_{1}, \tau_{2}\right)$ we have $(i, j)$-ind $X=n, n \geq$ 1 , then for each $k=\overline{0, n-1}$ the $\mathrm{BS}\left(X, \tau_{1}, \tau_{2}\right)$ contains a $p$-closed subset $Y$ such that $(i, j)$-ind $Y=k$.

Proof. It is enough to show that $X$ contains a $p$-closed subset $Y$ such that $(i, j)$-ind $Y=n-1$ since it is not difficult to see that $p-\mathrm{Cl}(Y) \subset p-\mathrm{Cl}(X)$ for every set $Y \in p-\mathrm{Cl}(X)$. Since $(i, j)$-ind $X>n-1$, there exist a point $x \in X$ and an $i$-neighborhood $U(x)$ such that for every $i$-open neighborhood $V(x)$ satisfying the condition $\tau_{j} \operatorname{cl} V(x) \subset U(x)$ we have $(i, j)$-ind $(j, i)$ Fr $V(x)>n-2$. On the other hand, since $(i, j)$-ind $X \leq n$, for the same $U(x)$ there exists an $i$-open neighborhood $V^{\prime}(x)$ such that $\tau_{j} \operatorname{cl} V^{\prime}(x) \subset U(x)$ and $(i, j)$-ind $(j, i)$-Fr $V^{\prime}(x) \leq n-1$. By the above arguments we also obtain $(i, j)$-ind $(j, i)$-Fr $V^{\prime}(x)>n-2$. Therefore for the $p$-closed subset $Y=$ $(j, i)-\operatorname{Fr} V^{\prime}(x)$ of $\left(X, \tau_{1}, \tau_{2}\right)$ we have $(i, j)$-ind $Y=n-1$. 
Corollary. If for a BS $\left(X, \tau_{1}<\tau_{2}\right)$ we have $(i, j)$-ind $X=n, n \geq 1$, then for each $k=\overline{0, n-1}$ the $\mathrm{BS}\left(X, \tau_{1}<\tau_{2}\right)$ contains a 2 -closed subset $Y$ such that $(i, j)$-ind $Y=k$.

Recall that a BS $\left(X, \tau_{1}, \tau_{2}\right)$ is $p$-normal if for every pair of disjoint sets $A, B$ in $X$, where $A$ is 1 -closed and $B$ is 2-closed, there exist a 2-open set $U \subset X$ and a 1-open $V \subset X$ such that $A \subset U, B \subset V$ and $U \cap V=\varnothing$ [15].

By analogy with the topological case, $\mathrm{BS}\left(X, \tau_{1}, \tau_{2}\right)$ is hereditarily $p$ normal if its every BsS is $p$-normal [6].

Theorem 3.1. If a $R$-p- $\mathrm{T}_{1}, d$-second countable and p-normal BS $\left(X, \tau_{1}, \tau_{2}\right)$ can be represented as the union of two BsS's $Y$ and $Z$ such that $(i, j)$-ind $Y \leq n$ and $(i, j)$-ind $Z \leq 0$, then $(i, j)$-ind $X \leq n+1$.

Proof. Let $x \in X$ be any point and $U(x) \in \tau_{i}$ its any neighborhood. Then by Theorem 2.10 below there exist disjoint sets $U \in \tau_{i}$ and $V \in \tau_{j}$ such that $x \in U, X \backslash U(x) \subset V$ and $(X \backslash(U \cup V)) \cap Z=\varnothing$. Clearly, $x \in U \subset U(x)$. But $(j, i)-\operatorname{Fr} U=\tau_{j} \operatorname{cl} U \cap(X \backslash U) \subset(X \backslash V) \cap(X \backslash U)=X \backslash(V \cup U) \subset X \backslash Z \subset Y$ and, by (2) of Proposition 2.1, $(i, j)$-ind $(j, i)$-Fr $U \leq n$. Thus $(i, j)$-ind $X \leq$ $n+1$.

Corollary. If a $R-p-T_{1}, d$-second countable and $p$-normal BS $\left(X, \tau_{1}, \tau_{2}\right)$ can be represented as the union of two BsS's $Y$ and $Z$ such that $p$-ind $Y \leq n$ and $p$-ind $Z \leq 0$, then $p$-ind $X \leq n+1$.

Theorem 3.2. If a $1-T_{1}, d$-second countable and p-normal $\mathrm{BS}\left(X, \tau_{1}<\right.$ $\left.\tau_{2}\right)$ can be represented as the union of two BsS's $Y$ and $Z$, where $p$-ind $Y=$ $p$-ind $Z=0$ and one of them is 1-closed, then $p$-ind $X=0$.

Proof. Let, for example $Y \in \operatorname{co} \tau_{1} \subset p-\mathrm{Cl}(X)$. Then $X \backslash Y \subset Z$ and, by (2) of Corollary 1 of Proposition 2.1, $p$-ind $(X \backslash Y)=0$, where $X \backslash Y \in \tau_{1}$. By Corollary of Proposition 0.1.3 in [11], the $\mathrm{BS}\left(X, \tau_{1}<\tau_{2}\right)$ is $p$-perfectly normal, i.e., $\tau_{i} \subset j-\mathcal{F}_{\sigma}(X)$ and hence $X \backslash Y \in 2-\mathcal{F}_{\sigma}(X)$, i.e., $X \backslash Y=\bigcup_{k=1}^{\infty} F_{k}$, where $F_{k} \in \operatorname{co} \tau_{2} \subset p-\mathrm{Cl}(X)$ and $p$-ind $F_{k}=0$ for every $k=\overline{1, \infty}$. On the other hand, $Y \in p-\mathrm{Cl}(X)$ and therefore $X=Y \cup(X \backslash Y)=Y \cup \bigcup_{k=1}^{\infty} F_{k}$. Thus it remains to use Corollary 2 of Theorem 2.11 below since $\left(X, \tau_{1}<\tau_{2}\right)$ is $1-T_{1}$ implies $\left(X, \tau_{1}<\tau_{2}\right)$ is $R-p-T_{1}$.

It is not difficult for one to verify that Theorem 2.2 remains valid if one of sets $Y$ and $Z$ is 1-open.

Theorem 3.3. Let a $R-p-T_{1}, d$-second countable and p-normal BS $\left(X, \tau_{1}, \tau_{2}\right)$ can be represented as the union of a sequence $F_{1}, F_{2}, \ldots$ of $p$-closed BsS's such that $(i, j)$-ind $F_{k} \leq n$ for each $k=\overline{1, \infty}$. Then $(i, j)$-ind $X \leq n$. 
Proof. We will apply induction with respect to the number $n$. For $n=$ 0 the theorem has already been proved in Corollary 2 of Theorem 2.11 below. Assume that the theorem holds for $n-1$ and prove it for $n$. Let $X=\bigcup_{k=1}^{\infty} F_{k}$, where $F_{k}=\tau_{1} \operatorname{cl} F_{k} \cap \tau_{2} \mathrm{cl} F_{k}$ and $(i, j)$-ind $F_{k} \leq n$ for each $k=\overline{1, \infty}$. By (2) of Corollary 2 of Proposition 2.1, choose for $k=\overline{1, \infty}$ a countable $i$-base $\mathcal{B}_{i}^{k}$ for the BsS $F_{k}$ such that $(i, j)$-ind $(j, i)$-Fr $U \leq n-1$ for every $U \in \mathcal{B}_{i}^{k}$. By the inductive assumption the $\operatorname{BsS} Y=\left\{\cup(j, i)\right.$-Fr $\left.U: U \in \bigcup_{k=1}^{\infty} \mathcal{B}_{i}^{k}\right\}$ of the BS $\left(X, \tau_{1}, \tau_{2}\right)$ satisfies the inequality $(i, j)$-ind $Y \leq n-1$. Now, by the second part of Corollary of Lemma 3.1.1 in [11], for each $k=\overline{1, \infty}$ the BsS $Z_{k}=F_{k} \backslash Y$ of a $\mathrm{BS}\left(F_{k}, \tau_{1}^{\prime}, \tau_{2}^{\prime}\right)$ satisfies the inequality $(i, j)$-ind $Z_{k} \leq 0$. Therefore, by Corollary 2 of Theorem 2.11 below, the BsS $Z=\bigcup_{k=1}^{\infty} Z_{k}=X \backslash Y$ of $\left(X, \tau_{1}, \tau_{2}\right)$ satisfies the inequality $(i, j)$-ind $Z \leq 0$ because it follows from the equalities $Z_{k}=F_{k} \backslash Y=F_{k} \cap Z=\left(\tau_{1} \operatorname{cl} F_{k} \cap \tau_{2} \operatorname{cl} F_{k}\right) \cap Z=\left(\tau_{1} \operatorname{cl} F_{k} \cap Z\right) \cap\left(\tau_{2} \operatorname{cl} F_{k} \cap Z\right)$ that all the $Z_{k}$ 's are $p$-closed in $Z$. Thus, by virtue of Theorem 2.1, we have $(i, j)$-ind $X \leq n$.

Corollary 3.1. Let a $R-p-T_{1}, d$-second countable and p-normal BS $\left(X, \tau_{1}, \tau_{2}\right)$ can be represented as the union of a sequence $F_{1}, F_{2}, \ldots$ of $p$-closed BsS's such that $p$-ind $F_{k} \leq n$ for each $k=\overline{1, \infty}$. Then $p$-ind $X \leq n$.

Corollary 3.2. Let a $1-T_{1}, d$-second countable and p-normal BS $\left(X, \tau_{1}<\right.$ $\left.\tau_{2}\right)$ can be represented as the union of a sequence $F_{1}, F_{2}, \ldots$ of $i$-closed sets, where $p$-ind $F_{k} \leq n$ for each $k=\overline{1, \infty}$, then $p$-ind $X \leq n$.

Proof. Follows directly from the inclusions co $\tau_{1} \subset \operatorname{co} \tau_{2} \subset p-\mathrm{Cl}(X)$.

Corollary 3.3. If a $R-p-T_{1}, d$-second countable and p-normal BS $\left(X, \tau_{1}, \tau_{2}\right)$ can be represented as the union of a sequence $F_{1}, F_{2}, \ldots$, where every $F_{k}$ is a countable union of $p$-closed sets, i.e., $F_{k}=\bigcup_{p=1}^{\infty} F_{p}^{k}$ and $p$-ind $F_{k} \leq n$ for each $k=\overline{1, \infty}$, then $p$-ind $X \leq n$.

Proof. By (2) of Proposition 2.1, $p$-ind $F_{p}^{k} \leq n$ for every $k=\overline{1, \infty}$, $p=\overline{1, \infty}$ and $X=\bigcup_{k=1}^{\infty} \bigcup_{p=1}^{\infty} F_{p}^{k}$.

Corollary 3.4. If a $1-T_{1}, d$-second countable and $p$-normal $\mathrm{BS}\left(X, \tau_{1}<\right.$ $\left.\tau_{2}\right)$ can be represented as the union of two BsS's $Y$ and $Z$, where $p$-ind $Y \leq n$, $p$-ind $Z \leq n$ and one of them is 1-closed or 1-open, then $p$-ind $X \leq n$.

Proof. Let $Y \in \tau_{1}$. Then $Y \in 2-\mathcal{F}_{\sigma}(X)$, so that $Y=\bigcup_{k=1}^{\infty} F_{k}$, where $F_{k} \in \operatorname{co} \tau_{2} \subset p-\operatorname{Cl}(X)$ for each $k=\overline{1, \infty}$. Since $X \backslash Y \subset Z$, we have $p$-ind $(X \backslash Y)$ 
$\leq n$. Clearly, $p$-ind $F_{k} \leq n$ for each $k=\overline{1, \infty}$ and therefore $p$-ind $X \leq n$ since $X=Y \cup(X \backslash Y)=\bigcup_{k=1}^{\infty} F_{k} \cup(X \backslash Y)$. The proof for the 1-closed set is similar.

Theorem 3.4. For a $R-p-\mathrm{T}_{1}, d$-second countable and $p$-normal BS $\left(X, \tau_{1}, \tau_{2}\right)$ we have: $(i, j)$-ind $X \leq n \Longleftrightarrow X$ can be represented as the union of two BsS's $Y$ and $Z$ such that $(i, j)$-ind $Y \leq n-1$ and $(i, j)$-ind $Z \leq 0$.

Proof. Let $(i, j)$-ind $X \leq n$. Then by (2) of Corollary 2 of Proposition 2.1, $X$ has a countable $i$-base $\mathcal{B}_{i}$ such that $(i, j)$-ind $(j, i)$-Fr $U \leq n-1$ for every $U \in \mathcal{B}_{i}$. By Theorem 2.3, the BsS $Y=\cup\left\{(j, i)\right.$-Fr $\left.U: U \in \mathcal{B}_{i}\right\}$ has $(i, j)$-ind $Y \leq n-1$ and, by the second part of Corollary of Lemma 3.1.1 in [11], $(i, j)$-ind $Z \leq 0$, where $Z=X \backslash Y$.

To complete the proof it suffices to apply Theorem 2.1.

Corollary. For a $R-p-T_{1}, d$-second countable and $p$-normal BS $\left(X, \tau_{1}, \tau_{2}\right)$ we have $p$-ind $X \leq n \Longleftrightarrow X$ can be represented as the union of two BsS's $Y$ and $Z$ such that $p$-ind $Y \leq n-1$ and $p$-ind $Z \leq 0$.

Theorem 3.5. If $\left(X, \tau_{1}, \tau_{2}\right)$ is a $R-p-T_{1}, d$-second countable and $p$ normal BS such that $p$-ind $X \leq n$, then for every pair $A \in \operatorname{co} \tau_{1}, B \in \operatorname{co} \tau_{2}$ and $A \cap B=\varnothing$ there exists a partition $T$ between $A$ and $B$ such that $p$-ind $T \leq$ $n-1$.

Proof. By Theorem 2.4, $X=Y \cup Z$, where $p$-ind $Y \leq n-1$ and $p$-ind $Z \leq 0$. Following Theorem 2.10 below, for $A$ and $B$ there exists a partition $T$ such that $T \cap Z=\varnothing$. Since $T \subset X \backslash Z \subset Y$, we have $p$-ind $T \leq$ $n-1$.

Theorem 3.6. Let $\left(X, \tau_{1}, \tau_{2}\right)$ be a $R-p-T_{1}$, hereditarily p-normal BS and $Y \subset X, Y$ be d-second countable and $p$-closed BsS such that $p$-ind $Y \leq n$. Then for every pair $A \in \operatorname{co} \tau_{1}, B \in \operatorname{co} \tau_{2}$ and $A \cap B=\varnothing$ there exists a partition $T$ between $A$ and $B$ such that $p-\operatorname{ind}(T \cap Y) \leq n-1$.

Proof. Since $p$-ind $Y \leq n$, by Corollary of Theorem 2.4, $Y=P \cup Q$, where $p$-ind $P \leq n-1, p$-ind $Q \leq 0$. Let $A \in \operatorname{co\tau } \tau_{1}, B \in \operatorname{co} \tau_{2}$ and $A \cap B=\varnothing$. Then $A \cap Y \in \operatorname{co} \tau_{1}^{\prime}, B \cap Y \in \operatorname{co} \tau_{2}^{\prime}$ in $\left(Y, \tau_{1}^{\prime}, \tau_{2}^{\prime}\right)$ and hence, there exists a partition $T^{\prime}$ between $A \cap Y$ and $B \cap Y$ such that $T^{\prime} \subset P$ and therefore $p$-ind $T^{\prime} \leq n-1$. By the second part of Lemma 2.3 below there exists a partition $T$ between $A$ and $B$ such that $T \cap Y \subset T^{\prime}$. Thus $p$-ind $(T \cap Y) \leq n-1$.

Theorem 3.7. For a d-second countable and p-normal BS $\left(X, \tau_{1}, \tau_{2}\right)$ we have: $(i, j)$-ind $X \leq n \Longleftrightarrow X$ has a countable $i$-network $\mathcal{N}_{i}$ such that $(i, j)$-ind $(j, i)-\operatorname{Fr} N \leq n-1$ for each $N \in \mathcal{N}_{i}$. 
Proof. Since any $i$-base is an $i$-network, by (2) of Corollary 2 of Proposition 2.1 it suffices to prove only that if an $i$-second countable BS has an $i$-network $\mathcal{N}=\left\{N_{k}\right\}_{k=1}^{\infty}$ such that $(i, j)$-ind $(j, i)$-Fr $N_{k} \leq n-1$ for every $k=$ $\overline{1, \infty}$, then $(i, j)$-ind $X \leq n$. Let $Y=\bigcup_{k=1}^{\infty}(j, i)$-Fr $N_{k}$ and $Z=X \backslash Y$. It follows from Theorem 2.3 that $(i, j)$-ind $Y \leq n-1$. We will show that $(i, j)$-ind $Z \leq 0$. For an arbitrary point $x \in Z$ and an $i$-neighborhood $U(x)$ there is $N_{k} \in \mathcal{N}_{i}$ such that $x \in N_{k} \subset U(x)$. Since $x \in X \backslash Y \subset X \backslash(j, i)-\operatorname{Fr} N_{k}=X \backslash\left(\tau_{j} \operatorname{cl} N_{k} \cap\right.$ $\left.\tau_{i} \operatorname{cl}\left(X \backslash N_{k}\right)\right)=\tau_{j} \operatorname{int}\left(X \backslash N_{k}\right) \cup \tau_{i}$ int $N_{k}$, we have $x \in V_{k}(x)=\tau_{i}$ int $N_{k} \subset$ $U(x)$. But $(j, i)$-Fr $V_{k}(x) \subset(j, i)$-Fr $N_{k}$ and hence $Z \cap(j, i)-\operatorname{Fr} V_{k}(x)=\varnothing$. Thus, by the second part of Corollary of Lemma 3.1.1 in [11], $(i, j)$-ind $Z \leq 0$. Therefore it remains to use Theorem 2.1.

Corollary. For a $R-p-T_{1}, d$-second countable and p-normal BS $\left(X, \tau_{1}, \tau_{2}\right)$ we have: $p$-ind $X \leq n \Longleftrightarrow X$ has a countable 1-network $\mathcal{N}_{1}$ and a countable 2-network $\mathcal{N}_{2}$ such that $(1,2)$-ind(2,1)-Fr $N \leq n-1$ for each $N \in \mathcal{N}_{1}$ and $(2,1)$-ind $(1,2)$-Fr $M \leq n-1$ for each $M \in \mathcal{N}_{2}$.

Recall that a point $x_{0}$ in a $\mathrm{BS}\left(X, \tau_{1}, \tau_{2}\right)$ is a point of tangency of topologies $\tau_{1}$ and $\tau_{2}$ if $\tau_{1}$ and $\tau_{2}$ coincide at $x_{0}$, i.e., if for each 1-open neighborhood $U\left(x_{0}\right)$ and each 2-open neighborhood $V\left(x_{0}\right)$ there exist a 2-open neighborhood $V^{\prime}\left(x_{0}\right)$ and a 1-open neighborhood $U^{\prime}\left(x_{0}\right)$ such that $V^{\prime}\left(x_{0}\right) \subset U\left(x_{0}\right)$ and $U^{\prime}\left(x_{0}\right) \subset V\left(x_{0}\right)[2]$.

Theorem 3.8. The following conditions are satisfied in a $\mathrm{BS}\left(X, \tau_{1}, \tau_{2}\right)$ :

(1) If $\tau_{1} \subset \tau_{2}$, then $(1,2)$-ind $X \leq 1$-ind $X \wedge 2$-ind $X \leq(2,1)$-ind $X$.

(2) If $\tau_{1} C \tau_{2}$, then 1 -ind $X \leq(1,2)$-ind $X$.

(3) If $\tau_{1} N \tau_{2}$, then $(2,1)$-ind $X \leq 2$-ind $X$.

(4) $x_{0} \bar{\in} \mathbf{n}_{1}(A)$ for each $A \in 2^{X} \Longleftrightarrow x_{0}$ is a point of tangency of $\tau_{1}$ and $\tau_{2} \Longrightarrow 2$-ind $_{x_{0}} X \leq(1,2)-$ ind $_{x_{0}} X \leq 1-$-ind $_{x_{0}} X \wedge 2$-ind $_{x_{0}} X \leq(2,1)$ $\operatorname{ind}_{x_{0}} X \leq 1-\operatorname{ind}_{x_{0}} X$.

Proof. (1) It is clear that the inequality $(1,2)$-ind $X \leq 1$-ind $X$ holds for 1 -ind $X=\infty$. Thus, assuming that 1 -ind $X=k<\infty$, we will show that $(1,2)$-ind $X \leq k$. For $k=-1$ the required inequality is obvious. Let us assume that this inequality is also correct for $k \leq n-1$ and prove it for $k=n$. Since 1-ind $X=n$, for every point $x \in X$ and its any 1-open neigbourhood $U(x)$ there exists an 1-open neighborhood $V(x)$ such that $\tau_{1} \operatorname{cl} V(x) \subset U(x)$ and 1-ind $(1-\operatorname{Fr} V(x)) \leq n-1$. Clearly, $\tau_{1} \subset \tau_{2}$ implies that $\tau_{2} \operatorname{cl} V(x) \subset \tau_{1} \operatorname{cl} V(x)$ so that $(2,1)-\operatorname{Fr} V(x) \subset 1-\operatorname{Fr} V(x)$. Hence by the monotonicity of the small 1-inductive dimension function we find that $1-\operatorname{ind}(2,1)-\operatorname{Fr} V(x) \leq n-1$ and by the inductive assumption $(1,2)$-ind(2,1)-Fr $V(x) \leq n-1$. Thus for every point $x \in X$ and its any 1-open neighborhood $U(x)$ there exists a 1-open 
neighborhood $V(x)$ such that $\tau_{2} \operatorname{cl} V(x) \subset U(x)$ and $(1,2)-\operatorname{ind}(2,1)-\operatorname{Fr} V(x) \leq$ $n-1$ so that $(1,2)$-ind $X \leq n$.

Furthermore, by analogy with the above reasoning we will prove that 2-ind $X \leq(2,1)$-ind $X$. Let us assume that this inequality holds for $(2,1)$-ind $X$ $=k \leq n-1$ and prove it for $k=n$. Since $(2,1)$-ind $X=n$, for every point $x \in$ $X$ and its any 2-open neighborhood $U(x)$ there exists a 2-open neighborhood $V(x)$ such that $\tau_{1} \operatorname{cl} V(x) \subset U(x)$ and $(2,1)$-ind(1,2)-Fr $V(x) \leq n-1$. But $\tau_{1} \subset \tau_{2}$ implies that $\tau_{2} \operatorname{cl} V(x) \subset \tau_{1} \operatorname{cl} V(x)$ so that 2-Fr $V(x) \subset(1,2)-\operatorname{Fr} V(x)$. Hence by the monotonicity of the small $(2,1)$-inductive dimension function we find that $(2,1)$-ind $(2-\operatorname{Fr} V(x)) \leq n-1$ and, by the inductive assumption, 2-ind (2-Fr $V(x)) \leq n-1$. Thus for every point $x \in X$ and its any 2open neighborhood $U(x)$ there exists a 2-open neighborhood $V(x)$ such that $\tau_{2} \operatorname{cl} V(x) \subset U(x)$ and 2-ind (2-Fr $\left.V(x)\right) \leq n-1$. Therefore 2-ind $X \leq n$.

(2) By analogy with (1) let us suppose that the required inequality is correct for $(1,2)$-ind $X=k \leq n-1$ and prove it for $k=n$. If $(1,2)$-ind $X=n$, then for every point $x \in X$ and its any 1-open neighborhood $U(x)$ there exists an 1-open neighborhood $V(x)$ such that $\tau_{2} \mathrm{cl} V(x) \subset U(x)$ and $(1,2)-\operatorname{ind}(2,1)$ Fr $V(x) \leq n-1$. But, by Definition 1.10, $\tau_{1} \operatorname{cl} V(x) \subset \tau_{2} \operatorname{cl} V(x)$ and therefore 1 -Fr $V(x) \subset(2,1)$-Fr $V(x)$. Hence by the monotonicity of the small $(1,2)$ inductive dimension function we have $(1,2)$-ind(1-Fr $V(x) \leq n-1$ and by the inductive assumption 1-ind(1-Fr $V(x) \leq n-1$. Thus for every point $x \in X$ and its any 1-open neighborhood $U(x)$ there exists an 1-open neighborhood $V(x)$ such that $\tau_{1} \operatorname{cl} V(x) \subset U(x)$ and 1-ind $(1-\operatorname{Fr} V(x)) \leq n-1$. Therefore 1 -ind $X \leq n$.

(3) Let us assume that the required inequality holds for 2-ind $X=k \leq$ $n-1$ and prove it for $k=n$. Since 2 -ind $X=n$, for every point $x \in X$ and its any 2-open neighborhood $U(x)$ there exists a 2-open neighborhood $V(x)$ such that $\tau_{2} \operatorname{cl} V(x) \subset U(x)$ and 2-ind $(2-\operatorname{Fr} V(x)) \leq n-1$. But, by Definition 1.14, $\tau_{1} \operatorname{cl} V(x) \subset \tau_{2} \operatorname{cl} V(x)$ and thus $(1,2)-\operatorname{Fr} V(x) \subset 2$-Fr $V(x)$. Hence because the small 2-inductive dimension function has the property of being monotone we find that 2 -ind(1,2)-Fr $V(x)) \leq n-1$ and by the inductive assumption $(2,1)$-ind(1,2)-Fr $V(x) \leq n-1$. Thus for every point $x \in X$ and its any 2-open neighborhood $U(x)$ there exists a 2-open neighborhood $V(x)$ such that $\tau_{1} \operatorname{cl} V(x) \subset U(x)$ and $(2,1)$-ind(1,2)-Fr $V(x) \leq n-1$. Therefore $(2,1)$-ind $X \leq n$.

(4) First let us prove the equivalence. If $x_{0} \in X$ is a tangency point of $\tau_{1}$ and $\tau_{2}$, then $x_{0} \in \tau_{1} \operatorname{cl} A \Longrightarrow x_{0} \in \tau_{2} \operatorname{cl} A$ for each $A \in 2^{X}$ so that $x_{0} \bar{\in} \mathbf{n}_{1}(A)$ for each $A \in 2^{X}$. Conversely, let $x_{0} \bar{\epsilon} \mathbf{n}_{1}(A)$ for each $A \in 2^{X}$. If $x_{0}$ is not a tangency point of $\tau_{1}$ and $\tau_{2}$, then there is a neighborhood $U\left(x_{0}\right) \in \tau_{2}$ such that for each neighborhood $V\left(x_{0}\right) \in \tau_{1}$ we have $V\left(x_{0}\right) \cap\left(X \backslash U\left(x_{0}\right)\right) \neq \varnothing$. 
Therefore $x_{0} \in \tau_{1} \operatorname{cl}\left(X \backslash U\left(x_{0}\right)\right)$. But $\left.x_{0} \bar{\epsilon}\left(X \backslash U\left(x_{0}\right)\right)=\tau_{2} \operatorname{cl}\left(X \backslash U\left(x_{0}\right)\right)\right)$, i.e., $x_{0} \in \mathbf{n}_{1}\left(X \backslash U\left(x_{0}\right)\right)$, which is impossible.

Now let $x_{0} \in X$ be a tangency point of $\tau_{1}$ and $\tau_{2}$. By (1) above it suffices to prove only that 2 -ind $x_{x_{0}} X \leq(1,2)$-ind $_{x_{0}} X \wedge(2,1)-$ ind $_{x_{0}} X \leq 1$-ind $x_{x_{0}} X$.

It is evident that the inequality 2 -ind $_{x_{0}} X \leq(1,2)$-ind $_{x_{0}} X$ holds for $(1,2)$-ind $_{x_{0}} X=\infty$. Thus, assuming that $(1,2)$-ind $_{x_{0}} X=k<\infty$, we will show that 2 -ind $_{x_{0}} X \leq k$. For $k=-1$ the required inequality is obvious. Let us suppose that this inequality is also correct for $k \leq n-1$ and prove it for $k=n$. Let $U\left(x_{0}\right) \in \tau_{2}$ be any neighborhood. Since $x_{0}$ is a tangency point of $\tau_{1}$ and $\tau_{2}$, there is a neighborhood $W\left(x_{0}\right) \in \tau_{2}$ such that $W\left(x_{0}\right) \subset U\left(x_{0}\right)$ and, by $(1,2)$-ind $_{x_{0}} X=n$, there is a neighborhood $V\left(x_{0}\right) \in \tau_{1}$ such that $\tau_{2} \operatorname{cl} V\left(x_{0}\right) \subset$ $W\left(x_{0}\right)$ and $(1,2)-\operatorname{ind}(2,1)-\operatorname{Fr} V\left(x_{0}\right) \leq n-1$. By (2) of Proposition 2.1 we have $(1,2)$-ind 2-Fr $V\left(x_{0}\right) \leq(1,2)$-ind $(2,1)-\operatorname{Fr} V\left(x_{0}\right) \leq n-1$ and, by the inductive assumption, 2-ind 2-Fr $V\left(x_{0}\right) \leq n-1$. Thus for each $U\left(x_{0}\right) \in \tau_{2}$ there is $V\left(x_{0}\right) \in \tau_{1} \subset \tau_{2}$ such that $\tau_{2} \operatorname{cl} V\left(x_{0}\right) \subset U\left(x_{0}\right)$ and 2-ind 2-Fr $V\left(x_{0}\right) \leq n-1$. Hence 2 -ind $x_{x_{0}} X \leq n$, i.e., 2 -ind $_{x_{0}} X \leq(1,2)$-ind $_{x_{0}} X$.

By analogy with the first case let us suppose that the required inequality is correct for $1-\operatorname{ind}_{x_{0}} X=k \leq n-1$ and prove it for $k=n$. Let $U\left(x_{0}\right) \in \tau_{2}$ be any neighborhood. Since $x_{0}$ is a tangency point of $\tau_{1}$ and $\tau_{2}$, there is a neighborhood $W\left(x_{0}\right) \in \tau_{1}$ such that $W\left(x_{0}\right) \subset U\left(x_{0}\right)$. But 1-ind $x_{0} X=n$ and therefore there is a neighborhood $V\left(x_{0}\right) \in \tau_{1}$ such that $\tau_{1} \operatorname{cl} V\left(x_{0}\right) \subset W\left(x_{0}\right)$ and 1-ind 1-Fr $V\left(x_{0}\right) \leq n-1$. By the monotonicity we have 1-ind (1,2)-Fr $V\left(x_{0}\right) \leq 1$-ind 1-Fr $V\left(x_{0}\right) \leq n-1$. On the other hand, by the inductive assumption, (2,1)-ind(1,2)-Fr $V\left(x_{0}\right) \leq n-1$. Thus for each $U\left(x_{0}\right) \in \tau_{2}$ there is $V\left(x_{0}\right) \in \tau_{1} \subset \tau_{2}$ such that $\tau_{1} \operatorname{cl} V\left(x_{0}\right) \subset$ $U\left(x_{0}\right)$ and $(2,1)$-ind(1,2)-Fr $V\left(x_{0}\right) \leq n-1$. Hence $(2,1)-\operatorname{ind}_{x_{0}} X \leq n$, i.e., $(2,1)-\operatorname{ind}_{x_{0}} X \leq 1-$ ind $_{x_{0}} X$.

Definition 3.3. Let $(A, B)$ be a pair of subsets of a BS $\left(X, \tau_{1}, \tau_{2}\right)$, where $A \in \operatorname{co} \tau_{j}, B \in \operatorname{co} \tau_{i}$ and $A \cap B=\varnothing$. Then the partition corresponding to $(A, B)$ is a p-closed set $T$ for which $X \backslash T=H_{1} \cup H_{2}, H_{i} \in \tau_{i} \backslash\{\varnothing\}, H_{1} \cap H_{2}=$ $\varnothing$ and $A \subset H_{i}, B \subset H_{j}$.

Remark 3.2. In the sequel, without loss of generality, we will sometimes consider a pair $(A, B)$, where $A \in \operatorname{co} \tau_{1}, B \in \operatorname{co} \tau_{2}$ and $A \cap B=\varnothing$. As in Remark 2.1, it is easy to verify that in a $\mathrm{BS}\left(X, \tau_{1}, \tau_{2}\right)$ the following conditions are satisfied for such a pair $(A, B)$ :

(1) If there exists a 2-open neighborhood $U(A)$ (a 1-open neighborhood $U(B)$ ) such that $\tau_{1} \operatorname{cl} U(A) \subset X \backslash B\left(\tau_{2} \operatorname{cl} U(B) \subset X \backslash A\right)$, then $(1,2)-\operatorname{Fr} U(A)$ $((2,1)-\operatorname{Fr} U(B))$ is the partition corresponding to $(A, B)$ in the sense of Definition 2.3. 
(2) If $T$ is the partition corresponding to $(A, B)$ in the sense of Definition 2.3, then $(j, i)-\operatorname{Fr} H_{i} \subset T$.

Definition 3.4. Let $\left(X, \tau_{1}, \tau_{2}\right)$ be a BS and $n$ denote a nonnegative integer. We say that:

(1) $(i, j)$-Ind $X=-1 \Longleftrightarrow X=\varnothing$.

(2) $(i, j)$-Ind $X \leq n$ if to every pair $(A, B)$, where $A \in \operatorname{co} \tau_{j}, B \in \operatorname{co} \tau_{i}$ and $A \cap B=\varnothing$, there corresponds a partition $T$ such that $(i, j)-\operatorname{Ind} T \leq n-1$.

(3) $(i, j)$-Ind $X=n$ if $(i, j)$-Ind $X \leq n$ and the inequality $(i, j)$-Ind $X \leq$ $n-1$ does not hold.

(4) $(i, j)$-Ind $X=\infty$ if the inequality $(i, j)$-Ind $X \leq n$ does not hold for any $n$.

Naturally, $p$-Ind $X \leq n \Longleftrightarrow(1,2)-\operatorname{Ind} X \leq n \wedge(2,1)-\operatorname{Ind} X \leq n$.

Lemma 3.1. Let $T$ be a partition in a $\mathrm{BS}\left(X, \tau_{1}, \tau_{2}\right)$ which corresponds to a pair of disjoint sets $(A, B)$, where $A \in \operatorname{co} \tau_{1}, B \in \operatorname{co} \tau_{2}$. If $Y \subset X$ is a pclosed set such that $A \cap Y \neq \varnothing \neq B \cap Y$, then the set $T^{\prime}=T \cap Y$ is the partition in a $\operatorname{BsS}\left(Y, \tau_{1}^{\prime}, \tau_{2}^{\prime}\right)$ corresponding to the pair $\left(A^{\prime}=A \cap Y, B^{\prime}=B \cap Y\right)$.

Proof. By condition $X \backslash T=H_{1} \cup H_{2}$, where $H_{i} \in \tau_{i} \backslash\{\varnothing\}, H_{1} \cap H_{2}=$ $\varnothing, A \subset H_{2}, B \subset H_{1}$. Hence $T=X \backslash\left(H_{1} \cup H_{2}\right)$.

Let us consider the set $Y \backslash T^{\prime}=Y \backslash\left(X \backslash\left(H_{1} \cup H_{2}\right)\right)=\left(Y \backslash\left(X \backslash H_{1}\right)\right) \cup$ $\left(Y \backslash\left(X \backslash H_{2}\right)\right)=\left(Y \cap H_{1}\right) \cup\left(Y \cap H_{2}\right)=H_{1}^{\prime} \cup H_{2}^{\prime}$, where $H_{i}^{\prime} \in \tau_{i}^{\prime} \backslash\{\varnothing\}, A^{\prime} \subset H_{2}^{\prime}$, $B^{\prime} \subset H_{1}^{\prime}$ and $H_{1}^{\prime} \cap H_{2}^{\prime}=\varnothing$.

Lemma 3.2. If $\left(A^{\prime}, B^{\prime}\right)$ is a pair of disjoint sets in a p-closed BsS $\left(Y, \tau_{1}^{\prime}, \tau_{2}^{\prime}\right)$ of a BS $\left(X, \tau_{1}, \tau_{2}\right)$ such that $A^{\prime} \in \operatorname{co} \tau_{1}^{\prime}$ and $B^{\prime} \in \operatorname{co} \tau_{2}^{\prime}$, then there exists a pair of disjoint sets $(A, B)$ in $\left(X, \tau_{1}, \tau_{2}\right)$ such that $A \in \operatorname{co} \tau_{1}, B \in \operatorname{co} \tau_{2}$, $A \cap Y=A^{\prime}$ and $B \cap Y=B^{\prime}$.

Proof. The fact that $Y$ is $p$-closed in $\left(X, \tau_{1}, \tau_{2}\right)$ implies $Y=\tau_{1} \operatorname{cl} Y \cap$ $\tau_{2} \operatorname{cl} Y$. Let $A^{\prime} \in \operatorname{co} \tau_{1}^{\prime}, B \in \operatorname{co} \tau_{2}^{\prime}$ in $\left(Y, \tau_{1}^{\prime}, \tau_{2}^{\prime}\right)$ and $A^{\prime} \cap B^{\prime}=\varnothing$. Then there are $A \in \operatorname{co} \tau_{1}^{\prime \prime}$ in $\left(\tau_{1} \mathrm{cl} Y, \tau_{1}^{\prime \prime}, \tau_{2}^{\prime \prime}\right)$ and $B \in \operatorname{co} \tau_{2}^{\prime \prime \prime}$ in $\left(\tau_{2} \operatorname{cl} Y, \tau_{1}^{\prime \prime \prime}, \tau_{2}^{\prime \prime \prime}\right)$ such that $A \cap Y=A^{\prime}$ and $B \cap Y=B^{\prime}$. It is evident that $A \in \operatorname{co} \tau_{1}, B \in \operatorname{co} \tau_{2}$ and $A \cap B=\varnothing$.

Corollary. A p-closed BsS of a p-normal BS is also p-normal.

Proposition 3.3. The following statements hold in a BS $\left(X, \tau_{1}, \tau_{2}\right)$ :

(1) If $(1,2)$-Ind $X$ or $(2,1)$-Ind $X$ is finite, then $\left(X, \tau_{1}, \tau_{2}\right)$ is p-normal.

(2) For every $p$-closed $\operatorname{BsS}\left(Y, \tau_{1}^{\prime}, \tau_{2}^{\prime}\right)$ of $\left(X, \tau_{1}, \tau_{2}\right)$ we have $(i, j)$-Ind $Y \leq$ $(i, j)$-Ind $X$.

(3) If $\left(X, \tau_{1}, \tau_{2}\right)$ is a $j-T_{1} \mathrm{BS}$, then $(i, j)$-ind $X \leq(i, j)$-Ind $X$. 
Proof. (1) Is obvious.

(2) It suffices to prove that $(i, j)$-Ind $X=\mathrm{k}$ implies $(i, j)$-Ind $Y \leq k$. This inequality is correct for $k=-1, k=\infty$. Let us assume that it is correct for $k \leq n-1$ and prove it for $k=n$. If $\left(A^{\prime}, B^{\prime}\right)$ is a pair of disjoint sets such that $A^{\prime} \in \operatorname{co} \tau_{j}^{\prime}$ and $B^{\prime} \in \operatorname{co} \tau_{i}^{\prime}$, then by Lemma 2.2 there exists a pair of disjoint sets $(A, B)$, where $A \in \operatorname{co} \tau_{j}, B \in \operatorname{co} \tau_{i}$ and $A \cap Y=A^{\prime}, B \cap Y=B^{\prime}$. Since $(i, j)$-Ind $X=n$, there exists a partition $T$ for $(A, B)$ such that $(i, j)$-Ind $T \leq$ $n-1$. But by Lemma $2.1, T^{\prime}=T \cap Y$ is the partition in $\left(Y, \tau_{1}^{\prime}, \tau_{2}^{\prime}\right)$ corresponding to the pair $\left(A^{\prime}, B^{\prime}\right)$. Hence, by the inductive assumption, $(i, j)$-Ind $T^{\prime} \leq n-1$ since $T^{\prime}$ is $p$-closed in $T$ so that $(i, j)$-Ind $Y \leq n$.

(3) Is clear.

Corollary 3.1. We have $p$-Ind $Y \leq p$-Ind $X$ for every $p$-closed BsS $\left(Y, \tau_{1}^{\prime}, \tau_{2}^{\prime}\right)$ of a $\mathrm{BS}\left(X, \tau_{1}, \tau_{2}\right)$ and therefore if $Y \in \operatorname{co} \tau_{1} \cup \operatorname{co} \tau_{2}$, then $p$-Ind $Y \leq$ $p$-Ind $X$. Moreover, if $\left(X, \tau_{1}, \tau_{2}\right)$ is $R-p-T_{1}$, then $p$-ind $X \leq p$-Ind $X$.

Corollary 3.2. Let $\left(X, \tau_{1}, \tau_{2}\right)$ be a BS and $n$ denote a nonnegative integer. Then $(i, j)$-Ind $X \leq n \Longleftrightarrow$ for any $j$-closed set $F$ and any $i$-neighborhood $U(F)$ there exists an $i$-open neighborhood $V(F)$ such that $\tau_{j} \operatorname{cl} V(F) \subset U(F)$ and $(i, j)-\operatorname{Ind}(j, i)-\operatorname{Fr} V(F) \leq n-1$.

The proof of this corollary repeats in the main that of Corollary 2 of Proposition 2.1, taking into account (2) of Remark 2.2 and (2) of Proposition 2.3 .

Corollary 3.3. If for a $\mathrm{BS}\left(X, \tau_{1}, \tau_{2}\right)$ we have $(i, j)$-Ind $X=n$ ( $p$-Ind $X=n), n \geq 1$, then for each $k=\overline{0, n-1}$, the $\mathrm{BS}\left(X, \tau_{1}, \tau_{2}\right)$ contains a $p$-closed $\operatorname{BsS}\left(Y, \tau_{1}^{\prime}, \tau_{2}^{\prime}\right)$ such that $(i, j)$-Ind $Y=k(p$-Ind $Y=k)$.

Proof. Is similar to the proof of Proposition 2.2 with Corollary 2 taken into account.

Proposition 3.4. The following equivalences hold for every BS $\left(X, \tau_{1}, \tau_{2}\right)$ :

$$
(1,2) \text {-Ind } X=0 \Longleftrightarrow(2,1)-\text { Ind } X=0 \Longleftrightarrow p \text {-Ind } X=0 \text {. }
$$

Proof. Let $(1,2)$-Ind $X=0, F \in \operatorname{co} \tau_{1}$ be any set and $U(F)$ be its any 2-open neighborhood. Then $X \backslash U(F) \subset X \backslash F$. Hence there exists a set $V \in$ $\tau_{1} \cap \operatorname{co} \tau_{2}$ such that $X \backslash U(F) \subset V \subset X \backslash F$ and therefore $F \subset X \backslash V \subset U(F)$, where $X \backslash V \in \tau_{2} \cap \operatorname{co} \tau_{1}$. Thus $(2,1)$-Ind $X=0$.

The inverse implication can be proved in a similar manner. 
Theorem 3.9. If for a d-second countable $\mathrm{BS}\left(X, \tau_{1}, \tau_{2}\right)$ the equality $p$-ind $X=0$ holds, then for every pair of disjoint sets $A \in \operatorname{co} \tau_{1}$ and $B \in \operatorname{co} \tau_{2}$ the empty set is a partition between them, i.e., there exists a set $V \in \tau_{2} \cap \operatorname{co} \tau_{1}$ such that $A \subset V$ and $B \subset X \backslash V$ and therefore $p$-Ind $X=0$.

Proof. Since $p$-ind $X=0$, for each point $x \in X$ there exists a set $U(x) \in \tau_{1} \cap \operatorname{co} \tau_{2}$ or a set $V(x) \in \tau_{2} \cap \operatorname{co} \tau_{1}$ such that

$$
(A \cap U(x)=\varnothing) \text { or }(B \cap V(x)=\varnothing) .
$$

It is evident that for the $p$-open cover $\mathcal{U}=\{\{U(x)\},\{V(x)\}\}_{x \in X}$ of $X$ there is a countable subfamily $\mathcal{U}^{\prime}=\left\{\left\{U\left(x_{k}\right): k=\overline{1, \infty}\right\},\left\{V\left(x_{p}\right): p=\right.\right.$ $\overline{1, \infty}\}\}$ which is also a $p$-open cover of $X$. The sets $U_{k}=U\left(x_{k}\right) \backslash \cup_{p<k} V\left(x_{p}\right)$ are 1open and 2-closed for each $k=\overline{1, \infty}$ and the sets $V_{p}=V\left(x_{p}\right) \backslash \cup_{k<p} U\left(x_{k}\right)$ are 2open and 1-closed for each $p=\overline{1, \infty}$. Moreover, $\mathcal{U}^{\prime \prime}=\left\{\left\{U_{k}: k=\overline{1, \infty}\right\},\left\{V_{p}\right.\right.$ : $p=\overline{1, \infty}\}\}$ is also a $p$-open cover of $X$. It is obvious that $U_{k} \cap A=\varnothing$ for each $k=\overline{1, \infty}$ and $V_{p} \cap B=\varnothing$ for each $p=\overline{1, \infty}$. Let $V=\cup\left\{V_{p}: A \cap V_{p} \neq \varnothing\right\}$. Then $A \subset V$. Since $B \cap V_{p}=\varnothing$ for each $p=\overline{1, \infty}$, we have $B \cap\left(\cup_{p=1}^{\infty} V_{p}\right)=\varnothing$ and therefore $B \subset X \backslash \bigcup_{p=1}^{\infty} V_{p} \subset X \backslash \cup\left\{V_{p}: A \cap V_{p} \neq \varnothing\right\}=X \backslash V$.

Corollary. If $\left(X, \tau_{1}, \tau_{2}\right)$ is $R-p-T_{1}$ and d-second countable, then $p$-Ind $X=0 \Longleftrightarrow p$-ind $X=0$.

Proof. By (3) of Proposition 2.3, $p$-Ind $X=0 \Longrightarrow p$-ind $X=0$. The inverse implication is an immediate consequence of Theorem 2.9 and Definition 2.4.

Lemma 3.3. Let $\left(Y, \tau_{1}^{\prime}, \tau_{2}^{\prime}\right)$ be a BsS of a hereditarily p-normal BS $\left(X, \tau_{1}, \tau_{2}\right)$ and $A \in \operatorname{co} \tau_{1}, B \in \operatorname{co} \tau_{2}, A \cap B=\varnothing$. Then for every partition $T^{\prime}$ in the $\operatorname{BsS}\left(Y, \tau_{1}^{\prime}, \tau_{2}^{\prime}\right)$ between $Y \cap \tau_{1} \mathrm{cl} V_{1}$ and $Y \cap \tau_{2} \mathrm{cl} V_{2}$, where $V_{1}$ and $V_{2}$ are 2-open and 1-open subsets of $X$ respectively such that $A \subset V_{1}, B \subset V_{2}$ and $\tau_{1} \mathrm{cl} V_{1} \cap \tau_{2} \mathrm{cl} V_{2}=\varnothing$, there exists a partition $T$ in $X$ between $A$ and $B$ which satisfies the inclusion $T \cap Y \subset T^{\prime}$.

If $\left(Y, \tau_{1}^{\prime}, \tau_{2}^{\prime}\right)$ is a p-closed BsS of a hereditarily p-normal BS $\left(X, \tau_{1}, \tau_{2}\right)$ and $A \in \operatorname{co} \tau_{1}, B \in \operatorname{co} \tau_{2}, A \cap B=\varnothing$, then for every partition $T^{\prime}$ in $\left(Y, \tau_{1}^{\prime}, \tau_{2}^{\prime}\right)$ between $A \cap Y$ and $B \cap Y$ there exists a partition $T$ in $X$ between $A$ and $B$ such that $T \cap Y \subset T^{\prime}$.

Proof. By Corollary of Theorem 0.2.1 and the Corollary of Proposition 0.1.2 in [11] there exist $V_{1} \in \tau_{2}, V_{2} \in \tau_{1}$ such that $A \subset V_{1}, B \subset V_{2}$ and $\tau_{1} \mathrm{cl} V_{1} \cap \tau_{2} \mathrm{cl} V_{2}=\varnothing$. On the other hand, since $\left(Y, \tau_{1}^{\prime}, \tau_{2}^{\prime}\right)$ is $p$-normal, 
there are $V_{1}^{\prime} \in \tau_{2}^{\prime}, V_{2}^{\prime} \in \tau_{1}^{\prime}$ such that $Y \cap \tau_{1} \operatorname{cl} V_{1} \subset V_{1}^{\prime}, Y \cap \tau_{2} \mathrm{cl} V_{2} \subset V_{2}^{\prime}$ and $V_{1}^{\prime} \cap V_{2}^{\prime}=\varnothing$. Let $T^{\prime}=Y \backslash\left(V_{1}^{\prime} \cup V_{2}^{\prime}\right)$. It is evident that

$$
A \cap \tau_{2} \operatorname{cl} V_{2}^{\prime}=\varnothing=B \cap \tau_{1} \operatorname{cl} V_{1}^{\prime} .
$$

Let us consider the $\operatorname{BsS}\left(V_{1}^{\prime} \cup V_{2}^{\prime}, \tau_{1}^{\prime \prime}, \tau_{2}^{\prime \prime}\right)$, where $V_{1}^{\prime} \in \tau_{2}^{\prime \prime}, V_{2}^{\prime} \in \tau_{1}^{\prime \prime}$. It is also clear that

$$
V_{1}^{\prime} \cap \tau_{2}^{\prime \prime} \operatorname{cl} V_{2}^{\prime}=\varnothing=V_{2}^{\prime} \cap \tau_{1}^{\prime \prime} \operatorname{cl} V_{1}^{\prime}
$$

Thus by (1) and (2) we have $\tau_{1} \operatorname{cl}\left(A \cup V_{1}^{\prime}\right) \cap \tau_{2} \operatorname{cl}\left(B \cup V_{2}^{\prime}\right)=\left(A \cup \tau_{1} \operatorname{cl} V_{1}^{\prime}\right) \cap$ $\left(B \cup \tau_{2} \mathrm{cl} V_{2}^{\prime}\right)=\varnothing$ and by Theorem 0.2 .1 in [11] there exist $H_{1} \in \tau_{2}, H_{2} \in \tau_{1}$ such that $A \cup V_{1}^{\prime} \subset H_{1}, B \cup V_{2}^{\prime} \subset H_{2}$ and $H_{1} \cap H_{2}=\varnothing$. The set $T=X \backslash\left(H_{1} \cup\right.$ $\left.H_{2}\right)$ is a partition in $X$ between $A$ and $B$ such that $T \cap Y=Y \backslash\left(H_{1} \cup H_{2}\right) \subset$ $Y \backslash\left(V_{1}^{\prime} \cup V_{2}^{\prime}\right)=T^{\prime}$, i.e., the first part is proved.

Now let $\left(Y=\tau_{1} \operatorname{cl} Y \cap \tau_{2} \operatorname{cl} Y, \tau_{1}^{\prime}, \tau_{2}^{\prime}\right)$ be a $p$-closed BsS of $\left(X, \tau_{1}, \tau_{2}\right)$ and $T^{\prime}=Y \backslash\left(V_{1}^{\prime} \cup V_{2}^{\prime}\right)$ any partition between $A \cap Y \in \operatorname{co} \tau_{1}^{\prime}$ and $B \cap Y \in \operatorname{co} \tau_{2}^{\prime}$ so that $A \cap Y \subset V_{1}^{\prime} \in \tau_{2}^{\prime}, B \cap Y \subset V_{2}^{\prime} \in \tau_{1}^{\prime}$ and $V_{1}^{\prime} \cap V_{2}^{\prime}=\varnothing$. Let $V_{1}^{\prime \prime} \in \tau_{2}^{\prime \prime}$ in $\left(\tau_{2} \mathrm{cl} Y, \tau_{1}^{\prime \prime}, \tau_{2}^{\prime \prime}\right)$ and $V_{2}^{\prime \prime \prime} \in \tau_{1}^{\prime \prime \prime}$ in $\left(\tau_{1} \mathrm{cl} Y, \tau_{1}^{\prime \prime \prime}, \tau_{2}^{\prime \prime \prime}\right)$ such that $A \cap \tau_{2} \mathrm{cl} Y \subset V_{1}^{\prime \prime}$, $B \cap \tau_{1} \operatorname{cl} Y \subset V_{2}^{\prime \prime \prime}$. If $V_{1} \in \tau_{2}$ and $V_{2} \in \tau_{1}$ are the sets for which $V_{1} \cap \tau_{2} \operatorname{cl} Y=V_{1}^{\prime \prime}$ and $V_{2} \cap \tau_{1} \operatorname{cl} Y=V_{2}^{\prime \prime \prime}$, then

$$
A \cap\left(\tau_{2} \operatorname{cl} Y \backslash V_{1}\right)=A \cap\left(\tau_{2} \operatorname{cl} Y \backslash V_{1}^{\prime \prime}\right)=\varnothing
$$

and

$$
B \cap\left(\tau_{1} \operatorname{cl} Y \backslash V_{2}\right)=B \cap\left(\tau_{1} \operatorname{cl} Y \backslash V_{2}^{\prime \prime \prime}\right)=\varnothing .
$$

Thus $A \subset X \backslash\left(\tau_{2} \operatorname{cl} Y \backslash V_{1}\right)=U \in \tau_{2}, B \subset X \backslash\left(\tau_{1} \operatorname{cl} Y \backslash V_{2}\right)=V \in \tau_{1}$. Since $A \cap B=\varnothing$, there exist $G \in \tau_{2}, H \in \tau_{1}$ such that $A \subset G, B \subset H$ and $G \cap H=\varnothing$. Let $M=U \cap G \in \tau_{2}, N=V \cap H \in \tau_{1}$. Then $A \subset M, B \subset N$ and, by (4) of Proposition 0.1.2 in [11], there exist $Q \in \tau_{2}, P \in \tau_{1}$ such that $A \subset Q \subset \tau_{1} \operatorname{cl} Q \subset U, B \subset P \subset \tau_{2} \operatorname{cl} P \subset V$ and $\tau_{1} \operatorname{cl} Q \cap \tau_{2} \operatorname{cl} P=\varnothing$. It is evident that $\tau_{1} \operatorname{cl} Q \cap Y \subset U \cap Y=Y \backslash\left(\tau_{2} \operatorname{cl} Y \backslash V_{1}\right)=V_{1}^{\prime}, \tau_{2} \operatorname{cl} P \cap Y \subset$ $V \cap Y=Y \backslash\left(\tau_{1} \operatorname{cl} Y \backslash V_{2}\right)=V_{2}^{\prime}$ so that $T^{\prime}=Y \backslash\left(V_{1}^{\prime} \cup V_{2}^{\prime}\right)$ is a partition between $\tau_{1} \operatorname{cl} Q \cap Y$ and $\tau_{2} \operatorname{cl} P \cap Y$. Hence, by the first part, there exists a partition $T$ between $A$ and $B$ in $X$ such that $T \cap Y \subset T^{\prime}$.

Theorem 3.10. Let $\left(X, \tau_{1}, \tau_{2}\right)$ be a $R-p-T_{1}, d$-second countable and $p$ normal BS, and $Y \subset X, p$-Ind $Y=0(\Longleftrightarrow p$-ind $Y=0)$. Then for every pair of disjoint sets $A \in \operatorname{co} \tau_{1}$ and $B \in \operatorname{co} \tau_{2}$ there exists a partition $T$ between $A$ and $B$ such that $T \cap Y=\varnothing$.

Proof. Let us consider $V_{1} \in \tau_{2}, V_{2} \in \tau_{1}$ such that $A \subset V_{1}, B \subset V_{2}$ and $\tau_{1} \mathrm{cl} V_{1} \cap \tau_{2} \mathrm{cl} V_{1}=\varnothing$. Then by Theorem 2.9 the empty set is a partition in $Y$ between $Y \cap \tau_{1} \mathrm{cl} V_{1}$ and $Y \cap \tau_{2} \mathrm{cl} V_{2}$. Thus it remains to use Lemma 2.3, since by Corollary of Proposition 0.1.3 in [11], $\left(X, \tau_{1}, \tau_{2}\right)$ is $p$-perfectly normal and hence hereditarily $p$-normal. 
Theorem 3.11. If a d-second countable and hereditarily p-normal BS $\left(X, \tau_{1}, \tau_{2}\right)$ can be represented as the union of a sequence $F_{1}, F_{2}, \ldots$ of $p$-closed sets, where $(1,2)$-Ind $F_{n}=0\left(\Longleftrightarrow(2,1)\right.$-Ind $F_{n}=0 \Longleftrightarrow p$-Ind $\left.F_{n}=0\right)$ for each $n=\overline{1, \infty}$, then $(1,2)$-Ind $X=0(\Longleftrightarrow(2,1)$-Ind $X=0 \Longleftrightarrow p$-Ind $X=$ $0)$.

Proof. Let $A \in \operatorname{co} \tau_{1}, B \in \operatorname{co} \tau_{2}$ and $A \cap B=\varnothing$. We will prove that there exist $G \in \tau_{2}, H \in \tau_{1}$ such that

$$
A \subset G, \quad B \subset H, \quad G \cap H=\varnothing \text { and } G \cup H=X,
$$

i.e., the empty set is a partition between $A$ and $B$.

Since $\left(X, \tau_{1}, \tau_{2}\right)$ is hereditarily $p$-normal and hence $p$-normal, by Corollary of Proposition 0.1.2 in [11], there exist $U_{0} \in \tau_{2}, V_{0} \in \tau_{1}$ such that

$$
A \subset U_{0}, \quad B \subset V_{0} \text { and } \tau_{1} \operatorname{cl} U_{0} \cap \tau_{2} \operatorname{cl} V_{0}=\varnothing .
$$

We will define inductively two sequences of 2-open and 1-open sets $U_{0}, U_{1}, \ldots$ and $V_{0}, V_{1}, \ldots$, respectively, satisfying the following conditions for each $k=\overline{0, \infty}$ :

$$
\begin{gathered}
U_{k-1} \subset U_{k}, \quad V_{k-1} \subset V_{k} \text { if } k \geq 1, \text { and } \tau_{1} \operatorname{cl} U_{k} \cap \tau_{2} \operatorname{cl} V_{k}=\varnothing, \\
F_{k} \subset U_{k} \cup V_{k}, \text { where } F_{0}=\varnothing .
\end{gathered}
$$

Clearly, the sets $U_{0}$ and $V_{0}$ defined above satisfy both conditions for $k=0$. Assume that the sets $U_{k}$ and $V_{k}$, satisfying (3) and (4), are defined for $k<p$. If $F_{p}=\tau_{1} \mathrm{cl} F_{p} \cap \tau_{2} \mathrm{cl} F_{p}$, then the sets $\tau_{1} \mathrm{cl} V_{p-1} \cap F_{p} \in \operatorname{co} \tau_{1}^{\prime}$ and $\tau_{2} \operatorname{cl} V_{p-1} \cap F_{p} \in \operatorname{co} \tau_{2}^{\prime}$ in $\left(F_{p}, \tau_{1}^{\prime}, \tau_{2}^{\prime}\right)$ are disjoint. Since $(1,2)$-Ind $F_{p}=0$, by virtue of Theorem 2.9 there exists a subset $V \in \tau_{2}^{\prime} \cap \operatorname{co} \tau_{1}^{\prime}$ such that $\tau_{1} \operatorname{cl} U_{p-1} \cap$ $F_{p} \subset V$ and $\tau_{2} \operatorname{cl} V_{p-1} \cap F_{p} \subset F_{p} \backslash V$. Since $\tau_{1} \operatorname{cl} V \subset \tau_{1} \operatorname{cl} F_{p}, \tau_{2} \operatorname{cl}\left(F_{p} \backslash V\right) \subset$ $\tau_{2} \operatorname{cl} F_{p}$ and $F_{p}=\tau_{1} \operatorname{cl} F_{p} \cap \tau_{2} \operatorname{cl} F_{p}$, we have $\tau_{1} \operatorname{cl} V \cap \tau_{2} \operatorname{cl}\left(F_{p} \backslash V\right)=\varnothing$.

Let $C=\tau_{1} \operatorname{cl} V \backslash \tau_{2} \operatorname{cl} V_{p-1}$ and $D=\tau_{2} \operatorname{cl}\left(F_{p} \backslash V\right) \backslash \tau_{1} \operatorname{cl} U_{p-1}$. Then $\left(\tau_{1} \mathrm{cl}\right.$ $C \cap D) \cup\left(C \cap \tau_{2} \mathrm{cl} D\right)=\varnothing$. Therefore by Theorem 0.2.1 in [11] there exist $U \in \tau_{2}, W \in \tau_{1}$ such that $C \subset U, D \subset W$ and $U \cap W=\varnothing$.

It is evident that $V \cap W=\varnothing,\left(F_{p} \backslash V\right) \cap U=\varnothing$ and consequently $\tau_{1} \operatorname{cl} V \cap W=\varnothing, \tau_{2} \operatorname{cl}\left(F_{p} \backslash V\right) \cap U=\varnothing$. Let $\Phi_{1}=\tau_{1} \operatorname{cl} U_{p-1} \cup\left(\tau_{1} \operatorname{cl} V \backslash W\right)$, $\Phi_{2}=\tau_{2} \operatorname{cl} V_{p-1} \cup\left(\tau_{2} \operatorname{cl}\left(F_{p} \backslash V\right) \backslash U\right)$. Then $\Phi_{i} \in \operatorname{co} \tau_{i}$ and $\Phi_{1} \cap \Phi_{2}=\varnothing$. Since $\left(X, \tau_{1}, \tau_{2}\right)$ is $p$-normal, there exist $U_{p} \in \tau_{2}, V_{p} \in \tau_{1}$ such that $\Phi_{1} \subset U_{p}$, $\Phi_{2} \subset V_{p}$ and $\tau_{1} \operatorname{cl} U_{p} \cap \tau_{2} \operatorname{cl} V_{p}=\varnothing$. The sets $\left(U_{p}\right)$ and $\left(V_{p}\right)$ satisfy (3) and (4) for $k=p$. Thus the construction of the sequences $U_{0}, U_{1}, \ldots$ and $V_{0}, V_{1}, \ldots$ is completed. It follows from (2), (3) and (4) that the unions $G=\bigcup_{p=1}^{\infty} U_{p}$ and $H=\bigcup_{p=1}^{\infty} V_{p}$ satisfy (1). 
Corollary 3.1. If a d-second countable and hereditarily p-normal BS $\left(X, \tau_{1}<\tau_{2}\right)$ can be represented as the union of a sequence $F_{1}, F_{2}, \ldots$ of $i$ closed sets, where $p$-Ind $F_{n}=0$ for every $n=\overline{1, \infty}$, then $p$-Ind $X=0$.

Proof. Is evident since co $\tau_{1} \subset \operatorname{co} \tau_{2} \subset p-\mathrm{Cl}(X)$.

Corollary 3.2. If a $R-p-T_{1}, d$-second countable and p-normal BS $\left(X, \tau_{1}, \tau_{2}\right)$ can be represented as the union of a sequence $F_{1}, F_{2}, \ldots$ of $p$-closed sets, where $p$-ind $F_{n}=0$ for every $n=\overline{1, \infty}$, then $p$-ind $X=0$.

Proof. Follows directly from Corollary of Theorem 2.9 and the fact that by Proposition 0.1 .3 in [11], $\left(X, \tau_{1}, \tau_{2}\right)$ is $p$-perfectly normal since it is $p$-regular and hence hereditarily $p$-normal.

Corollary 3.3. If a $\left(R-p-T_{1}\right) d$-second countable and hereditarily $p$ normal ( $p$-normal) BS $\left(X, \tau_{1}, \tau_{2}\right)$ can be represented as the union of a sequence $F_{1}, F_{2}, \ldots$, where every $F_{k}$ is a countable union of $p$-closed sets, i.e., $F_{k}=$ $\bigcup_{n=1}^{\infty} F_{n}^{k}$, and $p$-Ind $F_{k}=0\left(p\right.$-ind $\left.F_{k}=0\right)$ for each $k=\overline{1, \infty}$, then $p$-Ind $X=0$ $(p$-ind $X=0)$.

Proof. By Corollary 1 of Proposition 2.3, $p$-Ind $F_{n}^{k}=0$ for every $k=\overline{1, \infty}, n=\overline{1, \infty}$, and $X=\bigcup_{k=1}^{\infty} \bigcup_{n=1}^{\infty} F_{n}^{k}$. For $p$-ind $X$ it remains to use (2) of Proposition 2.1 and Corollary of Theorem 2.9.

Corollary 3.4. If a $R-p-T_{1}, d$-second countable and p-normal BS $\left(X, \tau_{1}<\tau_{2}\right)$ can be represented as the union of two BsS's $Y$ and $Z$, where $p$-Ind $Y=p$-Ind $Z=0$ and one of them is 1-open, then $p$-Ind $X=0$.

Proof. Let, for example, $Y \in \tau_{1}$. Then $X \backslash Y \in \operatorname{co} \tau_{1} \subset \operatorname{co} \tau_{2} \subset$ $p-\operatorname{Cl}(X), X \backslash Y \subset Z$ and by Corollary 1 of Proposition 2.3, $p-\operatorname{Ind}(X \backslash Y)=0$. Moreover, $\left(X, \tau_{1}<\tau_{2}\right)$ is $p$-perfectly normal so that $Y \in 2-\mathcal{F}_{\sigma}(X)$, i.e., $Y=\bigcup_{k=1}^{\infty} F_{k}$, where $F_{k} \in \operatorname{coc} \tau_{2} \subset p-\operatorname{Cl}(X)$ and, by (2) of Proposition 2.3, $p$-Ind $F_{k}=0$ for every $k=\overline{1, \infty}$. Therefore $X=Y \cup(X \backslash Y)=\bigcup_{k=1}^{\infty} F_{k} \cup(X \backslash Y)$ and it remains to use Corollary 1.

Theorem 3.12. for every $R-p-T_{1}, d$-second countable and $p$-normal BS $\left(X, \tau_{1}, \tau_{2}\right)$ we have $p$-ind $X=p$-Ind $X$.

Proof. By Corollary 1 of Proposition 2.3 it suffices to prove only that $p$-Ind $X \leq p$-ind $X$. It is evident that one can assume that $p$-ind $X<\infty$. We will apply induction with respect to $p$-ind $X$. Let $p$-ind $X=1$. Then by Theorem 2.5, for every disjoint pair of sets $A \in \operatorname{co} \tau_{1}$ and $B \in \operatorname{co} \tau_{2}$ there exists a partition $T$ between $A$ and $B$ such that $p$-ind $T \leq 0$. Hence by Corollary 
of Theorem 2.9, $p$-Ind $T \leq 0$, so that $p$-Ind $X \leq p$-ind $X$. Let us assume that the inequality is also correct for $k \leq n-1$ and prove it for $k=n$. Let $A \in \operatorname{co} \tau_{1}, B \in \operatorname{co} \tau_{2}$ and $A \cap B=\varnothing$. Then by Theorem 2.5 there exists a partition $T$ between $A$ and $B$ such that $p$-ind $T \leq n-1$. It follows from the inductive assumption that $p$-Ind $T \leq n-1$. Therefore $p$-Ind $X \leq n$, i.e., $p$-Ind $X \leq p$-ind $X$.

Corollary 3.1. Let a $R-p-T_{1}$, d-second countable and p-normal BS $\left(X, \tau_{1}, \tau_{2}\right)$ can be represented as the union of a sequence $F_{1}, F_{2}, \ldots$ of $p$-closed BsS's such that $p$-Ind $F_{k} \leq n$ for each $k=\overline{1, \infty}$. Then $p$-Ind $X \leq n$.

Proof. It is evident that $p$-ind $F_{k} \leq n$ for each $k=\overline{1, \infty}$ and by Corollary 1 of Theorem 2.3, $p$-ind $X \leq n$. Thus $p$-Ind $X \leq n$.

Corollary 3.2. Let $1-T_{1}, d$-second countable and p-normal BS $\left(X, \tau_{1}<\right.$ $\left.\tau_{2}\right)$ can be represented as the union of a sequence $F_{1}, F_{2}, \ldots$ of $i$-closed sets, where $p$-Ind $F_{k} \leq n$ for each $k=\overline{1, \infty}$. Then $p$-Ind $X \leq n$.

Corollary 3.3. If a $R-p-T_{1}, d$-second countable and p-normal BS $\left(X, \tau_{1}, \tau_{2}\right)$ can be represented as the union of a sequence $F_{1}, F_{2}, \ldots$, where every $F_{k}$ is a countable union of $p$-closed sets, i.e., $F_{k}=\bigcup_{p=1}^{\infty} F_{p}^{k}$ and $p$-Ind $F_{k} \leq n$ for each $k=\overline{1, \infty}$, then $p$-Ind $X \leq n$.

Proof. Since $p$-Ind $F_{k} \leq n$ and $F_{p}^{k} \in p-\operatorname{Cl}(X)$ for each $p=\overline{1, \infty}$, by (2) of Proposition 2.3, $p$-Ind $F_{p}^{k} \leq n$ for each $k=\overline{1, \infty}$ and $p=\overline{1, \infty}$. Thus, it remains to use Corollary 1.

Corollary 3.4. If a $1-T_{1}, d$-second countable and $p$-normal BS $\left(X, \tau_{1}<\right.$ $\left.\tau_{2}\right)$ can be represented as the union of two $\operatorname{BsS}$ 's $Y$ and $Z$, where $p$-Ind $Y \leq n$, $p$-Ind $Z \leq n$, and one of them is 1-open, then $p$-Ind $X \leq n$.

Proof. Since $\left(X, \tau_{1}, \tau_{2}\right)$ is $p$-perfectly normal, it can be assumed that $Y \in \tau_{1} \subset 2-\mathcal{F}_{\sigma}(X)$. Then $Y=\bigcup_{k=1}^{\infty} F_{k}$, where $F_{k} \in \operatorname{co} \tau_{2} \subset p-\mathrm{Cl}(X)$ for each $k=\overline{1, \infty}$ and by (2) of Proposition 2.3, $p$-Ind $F_{k} \leq n$ for each $k=\overline{1, \infty}$. On the other hand, $X \backslash Y \subset Z, X \backslash Y \in \operatorname{co} \tau_{1} \subset p-\mathrm{Cl}(X)$ and, by the same reasoning, $p-\operatorname{Ind}(X \backslash Y) \leq n$. But $X=Y \cup(X \backslash Y)=\bigcup_{k=1}^{\infty} F_{k} \cup(X \backslash Y)$ and it remains to use Corollary 1.

Theorem 3.13. The following conditions are satisfied in a $\mathrm{BS}\left(X, \tau_{1}, \tau_{2}\right)$ :

(1) If $\tau_{1}<_{C} \tau_{2}$, then 1 -Ind $X \leq(1,2)$-Ind $X$.

(2) If $\tau_{1}<_{N} \tau_{2}$, then 1 -Ind $X \leq(1,2)$-Ind $X \wedge(2,1)$-Ind $X \leq 2$-Ind $X$. 
Proof. (1) It is clear that the inequality 1 -Ind $X \leq(1,2)$-Ind $X$ holds for $(1,2)$-Ind $X=\infty$. Let us assume that $(1,2)$-Ind $X=k<\infty$ and show that 1-Ind $X \leq k$. For $k=-1$ the required inequality is obvious. Now let us assume that the inequality is correct for $k \leq n-1$ and prove it for $k=n$. Since $(1,2)$-Ind $X=n$, for every 2-closed set, in particular for every 1-closed set $F$ and its any 1-open neighborhood $U(F)$ there exists a 1-open neighborhood $V(F)$ such that $\tau_{2} \mathrm{cl} V(F) \subset U(F)$ and $(1,2)-\operatorname{Ind}(2,1)-\operatorname{Fr} V(F) \leq$ $n-1$. Since $\tau_{1}<_{C} \tau_{2}$ and $V(F) \in \tau_{1}$, by (2) of Corollary of Theorem 1.14, $\tau_{2} \operatorname{cl} V(F)=\tau_{1} \operatorname{cl} V(F)$ and thus $(2,1)-\operatorname{Fr} V(F)=1-\operatorname{Fr} V(F)$. Hence $(1,2)$ $\operatorname{Ind}(1-\operatorname{Fr} V(F)) \leq n-1$ and by the inductive assumption 1-Ind(1-Fr $V(F)) \leq$ $n-1$, i.e., for every 1-closed set $F$ and its any 1-open neighborhood $U(F)$ there exists a 1-open neighborhood $V(F)$ such that $\tau_{1} \mathrm{cl} V(F) \subset U(F)$ and 1-Ind $(1-\operatorname{Fr} V(F)) \leq n-1$. Therefore 1-Ind $X \leq n$.

(2) The first inequality is obvious by (1) and Corollary of Theorem 1.19. Furthermore, by analogy with the above reasoning we can prove that $(2,1)$ Ind $X \leq 2$-Ind $X$. Let us assume that the inequality holds for 2-Ind $X=$ $k \leq n-1$ and prove it for $k=n$. Since 2-Ind $X=n$, for every 2-closed set, in particular for every 1-closed set $F$ and its any 2-open neighborhood $U(F)$ there exists a 2-open neighborhood $V(F)$ such that $\tau_{2} \operatorname{cl} V(F) \subset U(F)$ and 2-Ind $(2-\operatorname{Fr} V(F)) \leq n-1$. Since $\tau_{1}<_{N} \tau_{2}$ and $V(F) \in \tau_{2}$, by Corollary of Theorem 1.19, $\tau_{2} \operatorname{cl} V(F)=\tau_{1} \operatorname{cl} V(F)$ and therefore 2-Fr $V(F)=$ $(1,2)$ - $\operatorname{Fr} V(F)$. Hence 2 -Ind $(1,2)-\operatorname{Fr} V(F) \leq n-1$ and by the inductive assumption $(2,1)-\operatorname{Ind}(1,2)-\operatorname{Fr} V(F) \leq n-1$. Thus for every 1-closed set $F$ and its any 2-open neighborhood $U(F)$ there exists a 2-open neighborhood $V(F)$ such that $\tau_{1} \operatorname{cl} V(F) \subset U(F)$ and $(2,1)-\operatorname{Ind}(1,2)-\operatorname{Fr} V(F) \leq n-1$. Therefore $(2,1)$-Ind $X \leq n$.

\section{Dynamics of Baire-Like Properties and Dimensions}

The objects of our final investigation are category notions, Baire-like properties and pairwise inductive dimensions in the context of $d$-continuous, $d$-closed, $(i, j)$-feebly continuous and $(i, j)$-feebly open functions.

In [9]-[11], the $(i, j)$-category requirements on $i$-open subsets with respect to $\left(X, \tau_{1}, \tau_{2}\right)$ and in itself as a BsS of $\left(X, \tau_{1}, \tau_{2}\right)$ form the basis of the determination of $(i, j)$-Baire spaces . Generally speaking, as distinct from the topological case, these arguments, are not the same. This distinction leads us to the definition of six different Baire-like properties which coincide when topologies are $S$-related in the sense of A. Todd [25]. It is necessary to note here that the pairwise Baire BS's introduced by C. Alegre, J. Ferer and V. Gregori in [1] are just the pairwise Baire BS's from [8] and are in fact 
the simplified modifications of the notions from [9]-[11] because they demand the $(i, j)$-category requirement of $i$-open subsets with respect to the whole BS. These pairwise Baire BS's called almost $(i, j)$-Baire spaces in [9]-[11] are studied in detail in [9], where an overwhelming majority of the results from [1] are obtained. Moreover, the notion of a pairwise fine BS in [1] is none other than a BS with the above-mentioned $S$-related topologies also studied and used for various purposes in [9]-[11].

Definition 4.1. An $(i, j)$-Baire space (briefly, an $(i, j)-B r S$ ) is a BS $\left(X, \tau_{1}, \tau_{2}\right)$ such that every nonempty $i$-open subset $U$ of $X$ is of $(i, j)$-second category [11].

This definition immediately implies that if $\left(X, \tau_{1}, \tau_{2}\right)$ is an $(i, j)$-BrS, then $X$ is of $(i, j)$ - $\mathcal{C}$ atg II.

Example 4.1. The natural $\mathrm{BS}\left(\mathbb{R}, \omega_{1}, \omega_{2}\right)$ is $(i, j)$-BrS since for every set $U \in \omega_{i} \backslash\{\varnothing\}$ the $\operatorname{BsS}\left(U, \omega_{1}^{\prime}, \omega_{2}^{\prime}\right)$ contains no nonempty $(i, j)$-nowhere dense sets. It is also clear that $\left(\mathbb{R}, \omega_{1}, \omega_{2}\right)$ is $i$ - BrS.

Therefore, in counterbalance to Remark 1.2 , the BS's $\left(\mathbb{R}, \omega_{1}\right)$ and $\left(\mathbb{R}, \omega_{2}\right)$ are both BrS's but $\omega_{1} S \omega_{2}$ is not correct.

Theorem 4.1. The following conditions are equivalent in a $\mathrm{BS}\left(X, \tau_{1}, \tau_{2}\right)$ :

(1) $U \in \tau_{i} \backslash\{\varnothing\} \Longrightarrow U \in(i, j)-\mathcal{C a t g}_{\mathrm{II}}(X)$.

(2) If $\left\{U_{n}\right\}_{n=1}^{\infty}$ is any countable family of subsets in $X$, where $U_{n} \in \tau_{j} \cap$ $i \mathcal{D}(X)$ for each $n=\overline{1, \infty}$, then $\bigcap_{n=1}^{\infty} U_{n} \in i-\mathcal{D}(X)$.

(3) $A \in(i, j)-\mathcal{C} a t g_{\mathrm{I}}(X) \Longrightarrow X \backslash A \in i-\mathcal{D}(X)$.

(4) If $\left\{F_{n}\right\}_{n=1}^{\infty}$ is any countable family of subsets in $X$, where $F_{n} \in \operatorname{co} \tau_{j} \cap$ $i-\mathcal{B} d(X)$ for each $n=\overline{1, \infty}$, then $\bigcup_{n=1}^{\infty} F_{n} \in i-\mathcal{B} d(X)$.

Definition 4.2. An almost $(i, j)$-Baire space (briefly, $A-(i, j)$-BrS) is $a \mathrm{BS}\left(X, \tau_{1}, \tau_{2}\right)$ for which one of the equivalent conditions (1)-(4) of Theorem 3.1 is satisfied [9].

The relations between Baire and almost Baire spaces are given in

Theorem 4.2. The following statements hold in $\mathrm{BS}\left(X, \tau_{1}, \tau_{2}\right)$ :

(1) $\left(X, \tau_{1}, \tau_{2}\right)$ is $i$ - $\mathrm{BrS} \Longleftrightarrow\left(X, \tau_{1}, \tau_{2}\right)$ is $A i$-BrS.

(2) $\left(X, \tau_{1}, \tau_{2}\right)$ is $(i, j)-\mathrm{BrS} \Longrightarrow\left(X, \tau_{1}, \tau_{2}\right)$ is $A-(i, j)-\mathrm{BrS}$.

In a $\mathrm{BS}\left(X, \tau_{1}<\tau_{2}\right)$

(3) $\left(X, \tau_{1}, \tau_{2}\right)$ is $(1,2)-\mathrm{BrS} \Longleftrightarrow\left(X, \tau_{1}, \tau_{2}\right)$ is $A-(1,2)-\mathrm{BrS} \Longrightarrow\left(X, \tau_{1}, \tau_{2}\right)$ is $1-\mathrm{BrS}$.

(4) $\left(X, \tau_{1}, \tau_{2}\right)$ is $2-\mathrm{BrS} \Longrightarrow\left(X, \tau_{1}, \tau_{2}\right)$ is $A-(2,1)-\mathrm{BrS}[9]$. 
Definition 4.3. Let $\left(X, \tau_{1}, \tau_{2}\right)$ and $\left(Y, \gamma_{1}, \gamma_{2}\right)$ be BS's. Then a function $f:\left(X, \tau_{1}, \tau_{2}\right) \rightarrow\left(Y, \gamma_{1}, \gamma_{2}\right)$ is said to be $i$-continuous ( $i$-open, $i$-closed) if the induced functions $f:\left(X, \tau_{i}\right) \rightarrow\left(Y, \gamma_{i}\right)$ are continuous (open, closed) [19], [22].

Theorem 4.3. Let a function onto $f:\left(X, \tau_{1}<\tau_{2}\right) \rightarrow\left(Y, \gamma_{1}<\gamma_{2}\right)$ be 1-continous and $d$-open, where $X$ has a countable 1-pseudobase and $Y$ is of $(1,2)$ - $\mathcal{C} a t g$ II. If there is a subset $Z \subset Y$ such that $Y \backslash Z \in(1,2)-\mathcal{C}$ atg $g_{\mathrm{I}}(Y)$ and $f^{-1}(z)$ is of $(1,2)-\mathcal{C}$ atg II for each point $z \in Z$, then $X$ is of $(1,2)$-Catg II.

Proof. Contrary, let $X$ is of $(1,2)-\mathcal{C} a t g I$. Then there is a sequence $\left\{F_{n}: \quad F_{n} \in \operatorname{co} \tau_{2} \cap(1,2)-\mathcal{N D}(X), n=\overline{1, \infty}\right\}$ such that $X=\bigcup_{n=1}^{\infty} F_{n}$. For each $n \in \mathbb{N}$ let $M\left(F_{n}\right)=\left\{y \in Y: \tau_{1}^{\prime} \operatorname{int}_{\left(f^{-1}(y), \tau_{1}^{\prime}, \tau_{2}^{\prime}\right)}\left(f^{-1}(y) \cap F_{n}\right) \neq \varnothing\right\}$. Let $\left\{U_{k}\right\}_{k=1}^{\infty}$ be a countable 1-pseudobase for $X$, and for each $n$ and $k$ let $M_{k}^{n}=\left\{y \in Y: \varnothing \neq f^{-1}(y) \cap U_{k} \subset F_{n}\right\}$. Since $f$ is $d$-open, we have $f\left(U_{k}\right) \in \gamma_{1}, U_{k} \cap\left(X \backslash F_{n}\right) \in \tau_{2}$ since $\tau_{1} \subset \tau_{2}, f\left(U_{k} \cap\left(X \backslash F_{n}\right)\right) \in \gamma_{2}$ and therefore $M_{k}^{n}=f\left(U_{k}\right) \backslash f\left(U_{k} \cap\left(X \backslash F_{n}\right)\right)$ is 2-closed in $f\left(U_{k}\right)$. If $\gamma_{1}$ int $M_{k}^{n} \neq \varnothing$, then $\varnothing \neq f^{-1}\left(\gamma_{1}\right.$ int $\left.M_{k}^{n}\right) \cap U_{k} \subset F_{n}$. For the set $f^{-1}\left(\gamma_{1}\right.$ int $\left.M_{k}^{n}\right) \cap U_{k} \in \tau_{1}$ there does not exist a set $V \in \tau_{2} \backslash\{\varnothing\}$ such that $V \subset f^{-1}\left(\gamma_{1}\right.$ int $\left.M_{k}^{n}\right) \cap U_{k}$ and $V \cap F_{n}=\varnothing$. Hence, by Corollary 1 of Theorem 1.1.1 in [11], we have $F_{n} \bar{\epsilon}(1,2)-\mathcal{N D}(X)$. Thus $\gamma_{1} \operatorname{int} M_{k}^{n}=\varnothing$. Since $M_{k}^{n} \in \operatorname{co} \gamma_{2}^{\prime}$ in $\left(f\left(U_{k}, \gamma_{1}^{\prime}, \gamma_{2}^{\prime}\right)\right.$, we have $\gamma_{1}$ int $\gamma_{2}^{\prime} \operatorname{cl} M_{k}^{n}=\varnothing$ and $f\left(U_{k}\right) \in \gamma_{1}$ implies $\gamma_{1}^{\prime}$ int $\gamma_{2}^{\prime} \operatorname{cl} M_{k}^{n}=\varnothing$ i.e., $M_{k}^{n} \in(1,2)-\mathcal{N} \mathcal{D}\left(f\left(U_{k}\right)\right)$ and, by (1) of Theorem 1.5.2 in [11], $M_{k}^{n} \in$ $(1,2)-\mathcal{N D}(Y)$. It is evident that $M\left(F_{n}\right)=\bigcup_{k=1}^{\infty} M_{k}^{n}$ for each $n \in \mathbb{N}$ so that $M\left(F_{n}\right) \in(1,2)-\mathcal{C} a t g_{\mathrm{I}}(Y)$ for each $n \in \mathbb{N}$ and therefore, by (1) of Theorem 1.1.3 in [11], $M=\bigcup_{n=1}^{\infty} M\left(F_{n}\right) \in(1,2)-\mathcal{C} a t g_{\mathrm{I}}(Y)$.

By condition, $Y$ is of $(1,2)-\mathcal{C}$ atg II and there is a subset $Z \subset Y$ such that $Y \backslash Z \in(1,2)-\mathcal{C} a t g_{\mathrm{I}}(Y)$. It is obvious that $Y \backslash M \in(1,2)-\mathcal{C}$ atg $g_{\mathrm{II}}(Y)$ since, by (1) of Theorem 1.1.3 in [11], the contrary means that $Y \in(1,2)-\mathcal{C} a t g \mathrm{I}$. Moreover, if $Y \backslash M \subset Y \backslash Z$, then by (1) of Theorem 1.1.3 in [11], $Y \backslash M \in$ $(1,2)-\mathcal{C} \operatorname{atg}_{\mathrm{I}}(Y)$, which is impossible. Hence $(Y \backslash M) \cap Z \neq \varnothing$. Let $z \in(Y \backslash M) \cap$ $Z$ be any point. It is evident that $\left\{F_{n} \cap f^{-1}(z)\right\}_{n=1}^{\infty}$ is a 2-closed cover of $f^{-1}(z)$. Since $f^{-1}(z)$ is of $(1,2)$ - $\mathcal{C}$ atg II, there is $k \in \mathbb{N}$ such $\tau_{1}^{\prime \prime} \operatorname{int}_{\left(f^{-1}(z), \tau_{1}^{\prime \prime}, \tau_{2}^{\prime \prime}\right)}$ $\left.\left(F_{k} \cap f^{-1}(z)\right) \neq \varnothing\right\}$. Thus $z \in M$. This contradiction completes the proof.

Corollary. Let a function onto $f:\left(X, \tau_{1}<\tau_{2}\right) \rightarrow\left(Y, \gamma_{1}<\gamma_{2}\right)$ be 1-continuous and d-open, where $X$ has a countable 1-pseudobase and $Y$ is $(1,2)$-BrS. If there is a subset $Z \subset Y$ such that $Y \backslash Z \in(1,2)-\mathcal{C}$ atg $\mathrm{I}_{\mathrm{I}}(Y)$ and $f^{-1}(z)$ is $(1,2)$-BrS for each point $z \in Z$, then $X$ is $(1,2)$-BrS. 
Proof. Let $U \in \tau_{1} \backslash\{\varnothing\}$ be any set. Then if $\left\{U_{k}\right\}_{k=1}^{\infty}$ is a countable 1-pseudobase of $X$, then $\left\{U_{k} \cap U\right\}_{k=1}^{\infty}$ is a countable 1-pseudobase of $U$. Moreover, $\left.f\right|_{U}: U \rightarrow f(U)$ is 1 -continuous and $d$-open. Since $\left(Y, \gamma_{1}, \gamma_{2}\right)$ is $(1,2)-\operatorname{BrS}$ and $f(U) \in \gamma_{1} \backslash\{\varnothing\}$, we have $f(U) \in(1,2)-\mathcal{C} a t g_{\mathrm{II}}(Y)$. Hence, by Corollary of Theorem 1.5.2 in [11], $f(U)$ is of $(1,2)-\mathcal{C}$ atg II. Suppose that $Z^{\prime}=Z \cap f(U)$. Then $f(U) \backslash Z^{\prime} \subset Y \backslash Z$ and by (1) of Theorem 1.1.3 in [11], $f(U) s l Z^{\prime} \in(1,2)-\mathcal{C} a t g_{\mathrm{I}}(Y)$. On the other hand, Corollary (2) of Theorem 1.5.1 in [11] implies that $f(U) \backslash Z^{\prime} \in(1,2)$ - $\mathcal{C} a t g_{\mathrm{I}} f(U)$ because $f(U) \in \gamma_{1}$. Now, let $z \in Z^{\prime}$ be any point. Then $\left(\left.f\right|_{U}\right)^{-1}(z)=f^{-1}(z) \cap U$ and since $f^{-1}(z)$ is $(1,2)-\operatorname{BrS}, f^{-1}(z) \cap U \in \tau_{1}^{\prime}$ in $\left(f^{-1}(z), \tau_{1}^{\prime}, \tau_{2}^{\prime}\right)$, by Corollary 1 of Theorem 4.1.3 in [11], $f^{-1}(z) \cap U$ is also $(1,2)$-BrS. Hence $f^{-1}(z) \cap U$ is $(1,2)-\mathcal{C} a t g$ II and the applying of Theorem 3.3 for $\left.f\right|_{U}: U \rightarrow f(U)$ gives that $U$ is of $(1,2)-\mathcal{C}$ atg II. Thus $\left(X, \tau_{1}, \tau_{2}\right)$ is $(1,2)$-BrS.

Finally, consider the following special modifications of feebly continuous and feebly open mappings.

Definition 4.4. A function $f:\left(X, \tau_{1}, \tau_{2}\right) \rightarrow\left(Y, \gamma_{1}, \gamma_{2}\right)$ is said to be $(i, j)$-feebly continuous $\left((i, j)\right.$-feebly open) if $V \in \gamma_{i} \backslash\{\varnothing\}\left(V \in \tau_{i} \backslash\{\varnothing\}\right)$ and $f^{-1}(V) \neq \varnothing$ imply that $\tau_{j}$ int $f^{-1}(V) \neq \varnothing\left(\gamma_{j}\right.$ int $\left.f(V) \neq \varnothing\right)$.

The classes of all $(i, j)$-feebly continuous $((i, j)$-feebly open) functions of $X$ to $Y$ are denoted by $(i, j)-\mathcal{F C}(X, Y)((i, j)-\mathcal{F} \mathcal{O}(X, Y))$.

It is easy to verify that the inclusions

$$
\begin{aligned}
& (2,1)-\mathcal{F C}(X, Y) \subset 1-\mathcal{F C}(X, Y) \\
& \text { and } \\
& \text { 2- } \mathcal{F C}(X, Y) \subset(1,2)-\mathcal{F C}(X, Y) \\
& (2,1)-\mathcal{F} \mathcal{O}(X, Y) \subset 1-\mathcal{F} \mathcal{O}(X, Y) \\
& \text { 2- } \mathcal{F O}(X, Y) \subset(1,2)-\mathcal{F O}(X, Y)
\end{aligned}
$$

hold for BS's $\left(X, \tau_{1}<\tau_{2}\right)$ and $\left(Y, \gamma_{1}<\gamma_{2}\right)$.

The statements below are the immediate consequences of the corresponding definitions.

Proposition 4.1. For a function $f:\left(X, \tau_{1}, \tau_{2}\right) \rightarrow\left(Y, \gamma_{1}, \gamma_{2}\right)$ the following conditions are satisfied:

(1) If $f$ is onto, then $f \in(i, j)-\mathcal{F} \mathcal{O}(X, Y) \Longleftrightarrow f^{-1}(j-\mathcal{D}(Y)) \subset i-\mathcal{D}(X)$.

(2) $f \in(i, j)-\mathcal{F C}(X, Y) \Longleftrightarrow f(j-\mathcal{D}(X)) \subset i-\mathcal{D}(Y)$.

Corollary. For a function $f:\left(X, \tau_{1}, \tau_{2}\right) \rightarrow\left(Y, \gamma_{1}, \gamma_{2}\right)$ the following conditions are satisfied:

(1) If $f$ is onto, then $f \in p-\mathcal{F O}(X, Y) \Longleftrightarrow\left(f^{-1}(1-\mathcal{D}(Y)) \subset 2-\mathcal{D}(X) \wedge\right.$ $\left.f^{-1}(2 \mathcal{D}(Y)) \subset 1-\mathcal{D}(X)\right)$.

(2) $f \in p-\mathcal{F C}(X, Y) \Longleftrightarrow(f(1-\mathcal{D}(X)) \subset 2-\mathcal{D}(Y) \wedge f(2-\mathcal{D}(X)) \subset 1-\mathcal{D}(Y))$. 
Theorem 4.4. If a function $f:\left(X, \tau_{1}<\tau_{2}\right) \rightarrow\left(Y, \gamma_{1}<\gamma_{2}\right)$ is oneto-one onto and $f \in(1,2)-\mathcal{F C}(X, Y) \cap(2,1)-\mathcal{F O}(X, Y)$, then $\left(X, \tau_{1}, \tau_{2}\right)$ is $A-(2,1)-\mathrm{BrS} \Longrightarrow\left(Y, \gamma_{1}, \gamma_{2}\right)$ is $(1,2)-\mathrm{BrS}$ and $\left(Y, \gamma_{1}, \gamma_{2}\right)$ is $A-(2,1)-\mathrm{BrS} \Longrightarrow$ $\left(X, \tau_{1}, \tau_{2}\right)$ is $(1,2)$-BrS.

Proof. Let $\left\{U_{n}\right\}_{n=1}^{\infty}$ be a sequence, where $U_{n} \in \gamma_{2} \cap 1-\mathcal{D}(Y)$ for each $n=\overline{1, \infty}$. We will prove that the sets $\tau_{1} \operatorname{int} f^{-1}\left(U_{n}\right) \in 2-\mathcal{D}(X)$ for each $n=\overline{1, \infty}$. Let $x_{0} \in X, n \in \mathbb{N}$ be arbitrarily fixed and $U\left(x_{0}\right) \in \tau_{2}$ any neighborhood. Since $f \in(2,1)-\mathcal{F O}(X, Y)$, we have $\gamma_{1}$ int $f\left(U\left(x_{0}\right)\right) \neq \varnothing$ so that there exists $V \in \gamma_{1} \backslash\{\varnothing\}$ such that $V \subset f\left(U\left(x_{0}\right)\right)$. The set $V \cap U_{n} \in \gamma_{2} \backslash\{\varnothing\}$ since $U_{n} \in 1-\mathcal{D}(Y)$ and $\gamma_{1} \subset \gamma_{2}$. But also $f \in(2,1)-\mathcal{F C}(X, y)$ and hence $\varnothing \neq W=$ $\tau_{1}$ int $f^{-1}\left(V \cap U_{n}\right)$. Therefore $W \subset f^{-1}\left(V \cap U_{n}\right) \subset f^{-1}\left(f\left(U\left(x_{0}\right)\right)=U\left(x_{0}\right)\right.$. It is clear that $W \subset \tau_{1} \operatorname{int} f^{-1}\left(U_{n}\right)$ and therefore $x_{0} \in \tau_{2} \mathrm{cl} \tau_{1} \operatorname{int} f^{-1}\left(U_{n}\right)$ since $U\left(x_{0}\right) \in \tau_{2}$ is an arbitrary neighborhood. Let $B_{n}=\tau_{1}$ int $f^{-1}\left(U_{n}\right)$. If $x_{0} \in X$ and $n \in \mathbb{N}$ are arbitrary fixed, then $B_{n} \in \tau_{1} \cap 2-\mathcal{D}(X)$ for each $n=\overline{1, \infty}$. On the other hand, $\left(X, \tau_{1}, \tau_{2}\right)$ is $A-(2,1)-\operatorname{BrS} \Longrightarrow \bigcap_{n=1}^{\infty} B_{n} \in 2-\mathcal{D}(X)$ and hence, by Proposition 5.1.5 from [9], $f\left(\bigcap_{n=1}^{\infty} B_{n}\right) \in 1-\mathcal{D}(Y)$ since $f \in$ $(2,1)-\mathcal{F C}(X, Y) \subset(1,2)-\mathcal{F C}(X, Y)$. Since $f\left(\bigcap_{n=1}^{\infty} B_{n}\right) \subset \bigcap_{n=1}^{\infty} f\left(B_{n}\right) \subset \bigcap_{n=1}^{\infty} U_{n}$, the set $\bigcap_{n=1}^{\infty} U_{n} \in 1-\mathcal{D}(Y)$ and thus $\left(Y, \gamma_{1}, \gamma_{2}\right)$ is $(1,2)$-BrS.

Finally, it is evident that $f \in(2,1)-\mathcal{F C}(X, Y) \Longleftrightarrow f^{-1}(2,1)-\mathcal{F O}(Y, X)$, $f \in(2,1)-\mathcal{F} \mathcal{O}(X, Y) \Longleftrightarrow f^{-1}(2,1)-\mathcal{F C}(Y, X)$ and therefore the rest is clear.

If a function $f:\left(X, \tau_{1}, \tau_{2}\right) \rightarrow\left(Y, \gamma_{1}, \gamma_{2}\right)$ is $d$-closed, $d$-continuous and $A \subset X, A \in \operatorname{co} \tau_{1} \cap \operatorname{co} \tau_{2}$, then $\left.f\right|_{A}$ is also $d$-closed and $d$-continuous. But in contrast to this fact, $\left.f\right|_{A}$ is not, generally speaking, $d$-closed, if $A \in p-\mathrm{Cl}(X)$.

Example 4.2. Let $X=\{a, b, c, d, e\}, \tau_{1}=\{\varnothing,\{a\}, X\}, \tau_{2}=\{\varnothing,\{b\}, X\}$, $Y=\{0,1\}, \gamma_{1}=\{\varnothing,\{0\}, Y\}$ and $\gamma_{2}=\{\varnothing,\{1\}, Y\}$. If $A=\{c, d, e\}$ and $f:\left(X, \tau_{1}, \tau_{2}\right) \rightarrow\left(Y, \gamma_{1}, \gamma_{2}\right)$ is defined as $f(a)=f(b)=0$ and $f(c)=f(d)=$ $f(e)=1$, then $f$ is $d$-closed but the restriction $\left.f\right|_{A}$, where $A \in p-\operatorname{Cl}(X)$, is not $d$-closed.

Theorem 4.5. Let $f:\left(X, \tau_{1}, \tau_{2}\right) \rightarrow\left(Y, \gamma_{1}, \gamma_{2}\right)$ be a d-closed and $d$ continuous function of a $R-p-T_{1}, d$-second countable and p-normal BS $X$ onto a $R-p-T_{1}, d$-second countable and p-normal $\mathrm{BS} Y$ such that for every set $A \in p-\mathrm{Cl}(X)$ the restriction $\left.f\right|_{A}: A \rightarrow f(A)$ is also $d$-closed and $d$ continuous. If there is an integer $k \geq 1$ such that $\left|f^{-1}(y)\right| \leq k$ for every $y \in Y$, then $(i, j)$-ind $Y \leq(i, j)$-ind $X+(k-1),(i, j)$-Ind $Y \leq(i, j)$-Ind $X+(k-1)$ and therefore $p$-ind $Y \leq p$-ind $X+(k-1), p$-Ind $Y \leq p$-ind $X+(k-1)$. 
Proof. By Theorem 2.13 it suffices to prove only the first inequality. We can suppose that $0 \leq(i, j)$-ind $X<\infty$ and we will apply induction with respect to the number $n+k$, where $n=(i, j)$-ind $X$. If $n+k=1$, then $k=1$ as $f$ is onto and hence $f$ is a $d$-homeomorphism and the theorem holds. Assume that the theorem holds whenever $n+k<m$, where $m \geq 2$ and consider a $d$-closed and $d$-continuous function $f: X \rightarrow Y$ such that $f(X)=Y$ and $n+k=m$.

Let $\mathcal{B}_{i}$ be a countable $i$-base of $\left(X, \tau_{1}, \tau_{2}\right)$ such that $(i, j)$-ind $(j, i)$ - $\operatorname{Fr} U \leq$ $n-1$ for every $U \in \mathcal{B}_{i}$. If $U \in \mathcal{B}_{i}$ is an arbitrary set, then $f \in d-\mathrm{Cl}(X, Y) \cap$ $d \mathcal{C}(X, Y)$ implies that

$$
\begin{gathered}
(j, i)-\operatorname{Fr} f(U)=\gamma_{j} \operatorname{cl} f(U) \cap \gamma_{i} \operatorname{cl}(Y \backslash f(U)) \subset f\left(\tau_{j} \operatorname{cl} U\right) \cap f(X \backslash U)= \\
=f(U \cup(j, i)-\operatorname{Fr} U) \cap f(X \backslash U)= \\
=(f(U) \cup f((j, i)-\operatorname{Fr} U)) \cap f(X \backslash U) \subset f((j, i)-\operatorname{Fr} U) \cup A,
\end{gathered}
$$

where $A=f(U) \cap f(X \backslash U)$. Since the restriction $\left.f\right|_{(j, i)-\operatorname{Fr} U}:(j, i)-\operatorname{Fr} U \rightarrow$ $f((j, i)-\operatorname{Fr} U)$ is $d$-closed and $d$-continuous, by the inductive assumption we have

$$
(i, j) \text {-ind } f((j, i)-\operatorname{Fr} U) \leq(n-1)+(k-1)=n+(k-2) .
$$

Assume that $A \neq \varnothing$ and consider the restriction $\left.f\right|_{f^{-1}(A)}=f_{A}$ : $f^{-1}(A) \rightarrow A$. It is well known that $f_{A}$ is $d$-closed and $d$-continuous. Moreover, the restriction $\left.f_{A}\right|_{(X \backslash U)}=f_{1}:(X \backslash U) \cap f^{-1}(A) \rightarrow A$ if also onto, $d$-closed and $d$-continuous.

The fibres of $f_{1}$ for each $y \in A$ have cardinality $\mid\left(f^{-1}(y) \mid \leq k-1\right.$ since, by condition, $\left|f^{-1}(y)\right| \leq k$ and $y \in A$ implies that $f_{1}^{-1}(y) \cap U=$ $f^{-1}(y) \cap U \neq \varnothing$. Therefore, it follows from the inductive assumption, that $(i, j)$-ind $A \leq n+(k-1)-1=n+(k-2)$, where $(i, j)$-ind $\left((X \backslash U) \cap f^{-1}(A)\right) \leq$ $(i, j)$-ind $X \leq n$.

Since $X$ is $p$-perfectly normal, i.e., $\tau_{i} \subset j-\mathcal{F}_{\sigma}(X)$, we have $U=\bigcup_{k=1}^{\infty} F_{k}$, where $F_{k} \in \operatorname{co} \tau_{j}$ for each $k=\overline{1, \infty}$. Hence $f(U)=\bigcup_{k=1}^{\infty} f\left(F_{k}\right) \in j-\mathcal{F}_{\sigma}(Y)$, $f(X \backslash U) \in \operatorname{co} \gamma_{i}$ so that $\left.A=f(U) \cap f(X \backslash U)=\bigcup_{k=1}^{\infty} f\left(F_{k}\right) \cap f(X \backslash U)\right)=\bigcup_{k=1}^{\infty} \Phi_{k}$, where each $\Phi_{k}=f\left(F_{k}\right) \cap f(X \backslash U)$ is p-closed. Hence by Corollary 3 of Theorem $2.3,(i, j)$-ind $(f(j, i)$ - $\operatorname{Fr} U \cup A) \leq n+(k-2)$. ¿From the latter inequality and (1) we obtain that $(i, j)$-ind $(j, i)$-Fr $f(U) \leq n+(k-2)$ for every $U \in \mathcal{B}_{i}$. The same inequality holds if $A=\varnothing$. It is not difficult to see that the family $\mathcal{N}_{i}=\left\{f(U): U \in \mathcal{B}_{i}\right\}$ is an $i$-network for $Y$ so that, by Theorem 2.7, $(i, j)$-ind $Y \leq n+k-1=(i, j)$-ind $X+(k-1)$.

Corollary. Let $f:\left(X, \tau_{1}<\tau_{2}\right) \rightarrow\left(Y, \gamma_{1}<\gamma_{2}\right)$ be a d-closed and $d$ continuous function of a $1-\mathrm{T}_{1}, d$-second countable and p-normal BS $X$ onto 
a 1-T $\mathrm{T}_{1}, d$-second countable and p-normal BS $Y$ such that for every set $A \in$ $\operatorname{co} \tau_{2}$ the restriction $\left.f\right|_{A}: A \rightarrow f(A)$ is 1-closed and 1-continuous. If there is an integer $k \geq 1$ such that $\left|f^{-1}(y)\right| \leq k$ for every $y \in Y$, then $p$-ind $Y \leq$ $p$-ind $X+(k-1)$ and $p$-Ind $Y \leq p$-Ind $X+(k-1)$.

\section{References}

[1] Alegre C., Ferrer J., Gregori V.: On pairwise Baire bitopological spaces, Publ. Math. Debrecen 55/1-2(1999), 3-15.

[2] Arhangelski A. V., Bokalo B. M.: General conception of the tangency of topologies, Abstr. Baku Inter. Conf. Top. Appl., Part II, 1987, p.19.

[3] Arhangelskiı̌ A. V., Ponomarev V. I.: Fundamentals of General Topology in Problems and Exercises (Russian), Nauka, Moscow, 1974.

[4] Ćirić M. D.: Dimension of bitopological spaces, Mathematica Balkanica 4.18 (1974), 99-105.

[5] Dvalishvili B. P.: On dimension of bitopological spaces, (Russian) Soob. Acad. Sci. Géorgian SSR 76, 1(1974), 49-52.

[6] Dvalishvili B. P.: On dimension and some other problems of the theory of bitopological spaces (Russian) Proc. Tbilisi Math. Inst. 56(1977), 15-51.

[7] Dvalishvili B. P.: Some principal questions of the theory of bitopological spaces, (Russian) Cand. of Science Thesis, Tbilisi State Univ., 1978.

[8] Dvalishvili B. P.: Bitopology and the Baire category theorem, Abstr. Tartu Conf. Problems of Pure Appl. Math., 1990, 90-93.

[9] Dvalishvili B. P.: Investigations of bitopologies and their applications, Dissertation for the Doctor of Science Degree, Tbilisi State Univ., 1994.

[10] Dvalishvili B. P.: Bitopological and algebraic structures in the context of Bairelike properties and generalized Boolean algebras, J. Math. Sci., (New York), 111 (2002), No. 1, 3227-3338.

[11] Dvalishvili B.: Bitopological Spaces: Theory, Relations with Generalized Algebraic Structures and Applications, Monograph (to appear).

[12] Fuglede B.: The quasi topology associated with a countably subadditive set function, Ann. Inst. Fourier, Grenoble 21, 1(1971), 123-169.

[13] Jelić M.: Some properties of dimension functions in bitopological spaces, (Russian) Mathematica Balkanica 4.54(1974), 309-311.

[14] Jelić M.: Some dimension functions in bitopological spaces, Mat. Vesnik 12(26)(1974), 38-42.

[15] Kelly J. C.: Bitopological spaces, Proc. London Math. Soc. (9)13(1963), 71-89. 
[16] Kuratowski K.: Sur l'opération $\bar{A}$ de l'Analysis Situs, Fund. Math. 3 (1922), 182-199.

[17] Kuratowski K.: Topology, vol. 1. (Russain) Mir, Moscow, 1966.

[18] Lukeš J., Malý J., Zajiček L.: Fine Topology Methods in Real Analysis and Potential Theory, Lecture Notes in Math., 1189, Springer-Verlag, 1986.

[19] Pervin W. J.: Connectedness in bitopological spaces, Nederl. Akad. Wetensh. Proc. Ser. A70.

[20] Reilly I. L.: Quasi-gauges, quasi-uniformities and bitopological spaces, Unpublished Ph. D. Thesis, Urbana-Champaign, Illinois Library, Univ. Illinois, 1970.

[21] Reilly I. L.: Zero-dimensional bitopological spaces, Nederl. Akad. Wetensh. Proc. Ser. A76, Indag Math. 35(1973), 127-131.

[22] Singal A. R.: Remarks on separation axioms, Gen. Topol. Rel. Mod. Anal. Algeb., Proc. Kanpur Topol. Conf., 1968, Prague, 1971, 265-296.

[23] Singal M. K., Singal A. R.: On some pairwise normality conditions in bitopological spaces, Publ. Math. 21(1974), No. 1-2, 71-81.

[24] Swart J.: Total disconnectedness in bitopological spaces and product bitopological spaces, Nederl. Akad. Wetensh. Proc. Ser. A74, Indag. Math. 33(1971), $135-145$.

[25] Todd A. R.: Quasiregular, pseudocomplete, and Baire spaces, Pacific J. Math. 95(1981), No. 1, 233-250.

[26] Weston J. D.: On the comparison of topologies, J. London Math. Soc. 32(1957), $342-354$.

Mechanics and Mathematics Faculty of I. Javakhishvili Tbilisi State University 2, University St., Tbilisi 380043 Georgia E-mails: acg@@wanex.net 